$5-2016$

\title{
Effective Integration of Ultra-Elliptic Solutions of the Focusing Nonlinear Schrödinger Equation
}

Otis C. Wright III

CedarvilleUniversity,wrighto@cedarville.edu

Follow this and additional works at: http://digitalcommons.cedarville.edu/

science_and_mathematics_publications

Part of the Physical Sciences and Mathematics Commons

\section{Recommended Citation}

Wright, Otis C. III, "Effective Integration of Ultra-Elliptic Solutions of the Focusing Nonlinear Schrödinger Equation" (2016). Science and Mathematics Faculty Publications. 326.

http://digitalcommons.cedarville.edu/science_and_mathematics_publications/326 


\title{
Effective integration of ultra-elliptic solutions of the focusing nonlinear Schrödinger equation
}

\author{
O.C. Wright III \\ Department of Science and Mathematics, Cedarville University, 251 N. Main St., Cedarville, OH 45314, United States
}

\section{H I G H L I G H T S}

- Kleinian ultra-elliptic function formulas for two-phase solutions of the focusing NLSE.

- Theta function formulas for two-phase solutions with explicit construction of parameters.

- Real loci of the Dirichlet eigenvalues parametrized by wave amplitude and wavenumber.

- Simple formulas for the extrema of the modulus of the two-phase solution.

\section{A R T I C L E I N F O}

\section{Article history:}

Received 26 May 2015

Received in revised form

11 January 2016

Accepted 1 March 2016

Available online 9 March 2016

Communicated by Peter David Miller

\section{Keywords:}

Nonlinear Schrödinger equation

Ultra-elliptic solutions

Two-phase solutions

\begin{abstract}
A B S T R A C T
An effective integration method based on the classical solution of the Jacobi inversion problem, using Kleinian ultra-elliptic functions and Riemann theta functions, is presented for the quasi-periodic twophase solutions of the focusing cubic nonlinear Schrödinger equation. Each two-phase solution with real quasi-periods forms a two-real-dimensional torus, modulo a circle of complex-phase factors, expressed as a ratio of theta functions associated with the Riemann surface of the invariant spectral curve. The initial conditions of the Dirichlet eigenvalues satisfy reality conditions which are explicitly parametrized by two physically-meaningful real variables: the squared modulus and a scalar multiple of the wavenumber. Simple new formulas for the maximum modulus and the minimum modulus are obtained in terms of the imaginary parts of the branch points of the Riemann surface.
\end{abstract}

(c) 2016 Elsevier B.V. All rights reserved.

\section{Introduction}

The focusing cubic nonlinear Schrödinger (NLS) equation is

$i p_{t}+p_{x x}+2|p|^{2} p=0$,

where $p(x, t)$ is a complex field exhibiting focusing, viz., modulationally unstable, behavior. The NLS equation is a well-known and thoroughly studied soliton equation applicable to a wide variety of problems, in which there is a simple balance between dispersive and nonlinear effects, ranging from shallow water waves to optical communication systems, see [1] and the references therein. The detailed study of two-phase, i.e., ultra-elliptic, solutions of the focusing NLS equation is important for understanding the smalldispersion limit near the gradient catastrophe [2] and, in particular, the generation of rogue waves near the gradient catastrophe [3]. Special classes of ultra-elliptic solutions of vector NLS equations have also been of much interest recently [4-7], including their modulation equations [8].

E-mail address: wrighto@cedarville.edu.
The construction of $\mathrm{N}$-phase or finite-gap solutions of the integrable focusing NLS equation (1) by algebro-geometric means has been studied extensively, a detailed history is contained in [9]. There are two main components to the algebro-geometric method of integration: (i) constructing a formula for the N-phase solution using a ratio of theta functions associated with the Riemann surface of the invariant spectral curve and (ii) satisfying the reality conditions imposed on the initial conditions of the solution by the real symmetry of the invariant spectral curve. The reality conditions must be satisfied by the invariant branch points of the Riemann surface and by the initial conditions of the moveable Dirichlet eigenvalues of the spectral problem.

The earliest studies by Kotlyarov, Its, and Čerednik [10-13] solved the first part of the integration problem using a classical solution to the Jacobi inversion problem, but satisfying the reality conditions remained at the level of an existence theorem. Dubrovin, Novikov, Previato, and others [9,14-21] were able to solve the problem of satisfying the reality conditions by studying the problem in the context of the image of the Dirichlet eigenvalues in the Jacobi variety of the Riemann surface. However, this more 
modern approach provides little information about the physicallymeaningful parameters in the solution, such as the amplitude of the wave and the wavenumber. Tracy and Chen [22,23] characterized the integration problem in a form amenable to numerical computations, but the reality conditions were still not satisfied explicitly. Exceptions to this situation are the explicit formulas for elliptic (one-phase) solutions of many integrable soliton equations (see $[1,24]$ and the references therein) and the two-phase solutions of the sine-Gordon equation $[25,26]$.

In this paper, the effective integration technique of Kamchatnov [1,24] is extended from the one-phase solution to the twophase solution of the focusing NLS equation. Using a classical solution to the Jacobi inversion problem for genus-two Riemann surfaces [27,28], a completely explicit formula for the two-phase solution of the focusing NLS equation is obtained [29]. The new formula for the solution is still a ratio of theta functions similar to previously known results, but the dependence of all the parameters in the solution on the initial conditions is now explicit. The integration method is effective because the reality conditions are explicitly satisfied in terms of the modulus and the wavenumber of the solution, and the parameters in the theta function formula for the solution are known explicitly in terms of the branch points and the correct initial conditions of the Dirichlet eigenvalues. Moreover, new simple formulas are obtained for the maximum modulus and the minimum modulus of the two-phase solution in terms of the imaginary parts of the branch points of the Riemann surface. Surprisingly, it appears that the simple dependence on the branch points of the Riemann surface of the maximum and the minimum of the complex modulus of the two-phase solution of the focusing NLS equation (1) was previously unknown in the literature until very recently [29].

\section{Lax pair of linear operators}

The initial steps in the integration of the two-phase solution of the NLS equation (1) follow the well-known pattern established by Kotlyarov, Its, and Dubrovin [10-12,14]. The integrability of the NLS equation is established through the equivalence of Eq. (1) and the commutation of a Lax pair of linear eigenvalue problems,

$\psi_{x}=\mathbb{U} \psi, \quad \psi_{t}=\mathbb{V} \psi$,

where

$$
\begin{aligned}
\mathbb{U} & =\left(\begin{array}{cc}
-i \lambda & i p \\
i p^{*} & i \lambda
\end{array}\right), \\
{[.3 i n] \mathbb{V} } & =\left(\begin{array}{cc}
-2 i \lambda^{2}+i|p|^{2} & 2 i \lambda p-p_{x} \\
2 i \lambda p^{*}+p_{x}^{*} & 2 i \lambda^{2}-i|p|^{2}
\end{array}\right),
\end{aligned}
$$

$p^{*}$ denotes the complex conjugate of $p$, and $\lambda$ is the spectral parameter in the inverse spectral theory of the integrable system.

The commutation of the Lax pair of linear operators (2) implies the NLS equation (1) in zero-curvature form

$\mathbb{U}_{t}-\mathbb{V}_{x}+[\mathbb{U}, \mathbb{V}]=0$,

where $[\mathbb{A}, \mathbb{B}]=A B-B A$ is the usual operation of matrix commutation. The stationary N-phase solutions of the NLS equation are, by definition, solutions of the stationary NLS hierarchy defined by

$\Psi_{x}=[\mathbb{U}, \Psi]$,

in which the solution matrix $\Psi$ is polynomial in the spectral parameter $\lambda$. The time-dependent $\mathrm{N}$-phase solutions are then obtained by explicitly constructing the compatible time-dependence of $\Psi$ such that

$\Psi_{t}=[\mathbb{V}, \Psi]$,

which, in turn, implies the zero-curvature representation of the NLS equation (4).
The commutation operators in Eqs. (5) and (6) imply that the trace of $\Psi$ is constant and so, without loss of generality, constant multiples of the identity matrix may be added to $\Psi$, and we may assume that the trace of $\Psi$ is zero. Furthermore, the commutation structure also implies that the characteristic polynomial of $\Psi$ is a constant, providing integrals of motion that enable the integration of the $\mathrm{N}$-phase solutions. Therefore a solution to Eq. (5) is constructed of the form,

$\Psi=\left(\begin{array}{cc}\Psi_{11} & \Psi_{12} \\ \Psi_{21} & -\Psi_{11}\end{array}\right)$

so that the Lax pair of Eqs. (5) and (6) becomes

$\Psi_{11 x}=-i p^{*} \Psi_{12}+i p \Psi_{21}$,

$\Psi_{12 x}=-2 i p \Psi_{11}-2 i \lambda \Psi_{12}$,

$\Psi_{21 x}=2 i p^{*} \Psi_{11}+2 i \lambda \Psi_{21}$,

$\Psi_{11 t}=-\left(2 i \lambda p^{*}+p_{x}^{*}\right) \Psi_{12}+\left(2 i \lambda p-p_{x}\right) \Psi_{21}$,

$\Psi_{12 t}=-2\left(2 i \lambda p-p_{x}\right) \Psi_{11}+2\left(-2 i \lambda^{2}+i|p|^{2}\right) \Psi_{12}$,

$\Psi_{21 t}=2\left(2 i \lambda p^{*}+p_{x}^{*}\right) \Psi_{11}+2\left(2 i \lambda^{2}-i|p|^{2}\right) \Psi_{21}$.

N-phase solutions, by definition, correspond to $\Psi$ which are polynomial in $\lambda$. Substitution of the series

$\Psi_{11}=1+\sum_{n=1}^{\infty} f_{n} \lambda^{-n}$

$\Psi_{12}=\sum_{n=1}^{\infty} g_{n} \lambda^{-n}$,

$\Psi_{21}=\sum_{n=1}^{\infty} h_{n} \lambda^{-n}$,

into the stationary equation (5), produces recursion relations for the entries of $\Psi$. It can be shown that the entries of $\Psi$ are differential polynomials in $p$ and $p^{*}[30]$.

\section{Two-phase solutions}

The previous formalism is now applied to two-phase solutions, viz., third-degree polynomial solutions $\Psi$ of Eq. (8). The most general such solution is

$$
\begin{aligned}
\Psi_{11}= & -i \lambda^{3}-i c_{2} \lambda^{2}+\left(\frac{1}{2} i|p|^{2}-i c_{1}\right) \lambda \\
& +\frac{1}{4}\left(p p^{* \prime}-p^{\prime} p^{*}\right)+\frac{1}{2} i c_{2}|p|^{2}-i c_{0}, \\
\Psi_{12}= & i p \lambda^{2}+\left(-\frac{1}{2} p^{\prime}+i c_{2} p\right) \lambda-\frac{1}{4} i p^{\prime \prime} \\
& -\frac{1}{2} i p|p|^{2}-\frac{1}{2} c_{2} p^{\prime}+i c_{1} p, \\
\Psi_{21}= & i p^{*} \lambda^{2}+\left(\frac{1}{2} p^{* \prime}+i c_{2} p^{*}\right) \lambda-\frac{1}{4} i p^{* \prime \prime} \\
& -\frac{1}{2} i p^{*}|p|^{2}+\frac{1}{2} c_{2} p^{* \prime}+i c_{1} p^{*},
\end{aligned}
$$

where $c_{0}, c_{1}, c_{2} \in \mathbb{R}$ are constants of integration. The solution $\Psi$ in Eq. (10) has a real symmetry which must be satisfied so that the potential $p$ in the linear spectral problem of the Lax pair actually solves the scalar NLS equation (1), instead of a complexified pair of coupled NLS equations.

Theorem 1 (Reality Condition).

$\Psi_{12}(\lambda)=-\left(\Psi_{21}\left(\lambda^{*}\right)\right)^{*}$, 
and, hence, the two roots $\mu_{1}$ and $\mu_{2}$ of $\Psi_{21}(\lambda)=0$ are the complex conjugates of the two roots of $\Psi_{12}(\lambda)=0$. In particular,

$$
\begin{aligned}
\Psi_{11}= & -i \lambda^{3}-i c_{2} \lambda^{2}+\left(\frac{1}{2} i v_{1}-i c_{1}\right) \lambda+\frac{1}{4} i v_{2} \\
& +\frac{1}{2} i c_{2} v_{1}-i c_{0},
\end{aligned}
$$

$\Psi_{12}=i p\left(\lambda-\mu_{1}\right)\left(\lambda-\mu_{2}\right)$,

$\Psi_{21}=i p^{*}\left(\lambda-\mu_{1}^{*}\right)\left(\lambda-\mu_{2}^{*}\right)$,

where $v_{1}, v_{2} \in \mathbb{R}$ are real variables,

$v_{1}=|p|^{2} \geq 0$,

$v_{2}=i\left(p^{*} p^{\prime}-p p^{* \prime}\right)$.

In the context of water-wave applications of the NLS equation, $v_{1}$ is the square of the amplitude of the wave, and $v_{2}$ is a constant multiple of the spatial wavenumber. However, in the opticalpulse setting, $v_{1}$ represents the squared modulus of the intensity of the lightwave, and $v_{2}$ is a constant multiple of the temporal wavenumber. The two roots $\mu_{1}, \mu_{2} \in \mathbb{C}$ of the equation $\Psi_{12}=$ 0 are analogous to the Dirichlet eigenvalues of the KdV spectral problem and, hence, are referred to as Dirichlet eigenvalues in this context. The solution $p(x, t)$ of the NLS equation (1) is recovered from the Dirichlet eigenvalues by the trace formulas.

Lemma 1 (Trace Formulas). The Dirichlet eigenvalues $\mu_{1}$ and $\mu_{2}$ satisfy the trace formulas,

$$
\begin{aligned}
\mu_{1}+\mu_{2} & =-\frac{1}{2} i \frac{p^{\prime}}{p}-c_{2}, \\
\mu_{1} \mu_{2} & =-\frac{1}{4} \frac{p^{\prime \prime}}{p}-\frac{1}{2}|p|^{2}+\frac{1}{2} i c_{2} \frac{p^{\prime}}{p}+c_{1} .
\end{aligned}
$$

Also

$v_{2}=-2 v_{1}\left(\mu_{1}+\mu_{2}+\mu_{1}^{*}+\mu_{2}^{*}+2 c_{2}\right)$,

and

$p^{\prime}=2 i p\left(\mu_{1}+\mu_{2}+c_{2}\right)$,

$p^{\prime \prime}=-2 p\left(2 \mu_{1} \mu_{2}+v_{1}+2 c_{2} \mu_{1}+2 c_{2} \mu_{2}+2 c_{2}^{2}-2 c_{1}\right)$.

The evolution of the Dirichlet eigenvalues is governed by the Dubrovin equations [14] obtained by substitution of Eq. (12) into the second and fifth equations of (8), differentiating, and substituting $\lambda=\mu_{1}$ or $\lambda=\mu_{2}$.

Lemma 2 (Dubrovin Equations). The Dirichlet eigenvalues $\mu_{1}$ and $\mu_{2}$ satisfy the following system of equations,

$\frac{\partial \mu_{1}}{\partial x}=2 \frac{\Psi_{11}\left(\mu_{1}\right)}{\mu_{1}-\mu_{2}}$

$\frac{\partial \mu_{2}}{\partial x}=2 \frac{\Psi_{11}\left(\mu_{2}\right)}{\mu_{2}-\mu_{1}}$

$\frac{\partial \mu_{1}}{\partial t}=-2\left(\mu_{2}+c_{2}\right) \frac{\partial \mu_{1}}{\partial x}$,

$\frac{\partial \mu_{2}}{\partial t}=-2\left(\mu_{1}+c_{2}\right) \frac{\partial \mu_{2}}{\partial x}$.

The Dirichlet eigenvalues lie on trajectories in the complex plane determined by the Dubrovin differential equations (17) and the initial conditions. However, not all initial conditions satisfy the reality conditions, viz., the integrals of motion of the Dubrovin equations and the constraint that the zeros of $\Psi_{12}$ and $\Psi_{21}$ are complex conjugates of each other. The allowed initial conditions, viz., the allowed trajectories, are not known a priori (unlike the KdV case in which the Dirichlet eigenvalues must lie on certain intervals of the real line determined by the spectral problem), instead the trajectories must be determined in order to construct two-phase solutions from the Dubrovin equations.

At this juncture, we depart from the approach of Its, Kotlyarov, Dubrovin, and others [9-21] and, instead, adopt the effective integration method used by Kamchatnov [1,24] for one-phase (elliptic) solutions. In this method it is observed that the Dubrovin equations imply that the $\mu$-trajectories of the Dirichlet eigenvalues are parametrized by the two real variables $v_{1}$ and $v_{2}$. Therefore, by finding the algebraic dependence of the $\mu$-trajectories on $v_{1}$ and $v_{2}$, the reality conditions can be satisfied explicitly.

The differential equations satisfied by $v_{1}$ and $v_{2}$ come from the $x$-flow and $t$-flow of the Lax pair for $\Psi$ by substitution of Eqs. (12) into Eqs. (8).

Lemma 3. The real variables $v_{1}$ and $v_{2}$ satisfy the following system of equations,

$$
\begin{aligned}
\frac{\partial v_{1}}{\partial x}= & 2 i v_{1}\left(\mu_{1}+\mu_{2}-\mu_{1}^{*}-\mu_{2}^{*}\right) \\
\frac{\partial v_{2}}{\partial x}= & 4 i v_{1}\left(\mu_{1}^{*} \mu_{2}^{*}-\mu_{1} \mu_{2}\right)-2 c_{2} \frac{\partial v_{1}}{\partial x} \\
\frac{\partial v_{1}}{\partial t}= & \frac{\partial v_{2}}{\partial x} \\
\frac{\partial v_{2}}{\partial t}= & 8 i v_{1}\left(\left(\mu_{1}-\mu_{1}^{*}\right)\left|\mu_{2}\right|^{2}+\left(\mu_{2}-\mu_{2}^{*}\right)\left|\mu_{1}\right|^{2}\right) \\
& -4 c_{2} \frac{\partial v_{2}}{\partial x}-4 c_{2}^{2} \frac{\partial v_{1}}{\partial x}
\end{aligned}
$$

If $p(x, t)$ is a smooth bounded quasi-periodic two-phase solution of the NLS equation (1), then $v_{1}(x, t)=|p(x, t)|^{2}$ must oscillate on finite intervals and, hence, it must have relative extrema as a function of $x$ and $t$. The relative extrema must occur at critical points. Moreover, the critical points can be characterized as distinguished points on the trajectories of the Dirichlet eigenvalues.

Lemma 4. If $p(x, t)$ is a smooth bounded quasi-periodic two-phase solution of the NLS equation (1), then $v_{1}(x, t)=|p(x, t)|^{2}$ must have relative extrema as a function of $x$ and $t$. Critical points of $v_{1}(x, t)$ occur at distinguished values of the Dirichlet eigenvalues. In particular, for $v_{1}>0$, Eqs. (18) imply that

$$
\frac{\partial v_{1}}{\partial x}=\frac{\partial v_{1}}{\partial t}=0 \Leftrightarrow \text { (i) } \mu_{1}=\mu_{1}^{*}, \mu_{2}=\mu_{2}^{*} \text { or (ii) } \mu_{2}^{*}=\mu_{1} \text {. }
$$

Theorem 2. If $p(x, t)$ is a smooth bounded quasi-periodic two-phase solution of the NLS equation (1), then $v_{1}(x, t)=|p(x, t)|^{2}$ must oscillate on an interval of non-negative values whose endpoints are relative extrema of $v_{1}(x, t)$. If $v_{1}>0$ and $\mu_{1} \neq \mu_{2}$, then $v_{1}$ has a relative extremum only if $\mu_{1}=\mu_{1}^{*}$ and $\mu_{2}=\mu_{2}^{*}$.

Proof. Lemma 4 establishes the possible locations of relative extrema. Explicit calculation using the Dubrovin equations (17) and (18) shows that if $\mu_{1} \neq \mu_{2}$ and (i) $\mu_{1}=\mu_{1}^{*}$ and $\mu_{2}=\mu_{2}^{*}$ or (ii) $\mu_{1}=\mu_{2}^{*}$, then

$\frac{\partial^{2} v_{1}}{\partial x^{2}} \frac{\partial^{2} v_{1}}{\partial t^{2}}-\left(\frac{\partial^{2} v_{1}}{\partial x \partial t}\right)^{2}=256 v_{1}^{2} \Psi_{11}\left(\mu_{1}\right) \Psi_{11}\left(\mu_{2}\right) \in \mathbb{R}$.

However, if $\mu_{1} \neq \mu_{2}$ and $\mu_{1}=\mu_{2}^{*}$ and $v_{1}>0$, then

$$
\frac{\partial^{2} v_{1}}{\partial x^{2}} \frac{\partial^{2} v_{1}}{\partial t^{2}}-\left(\frac{\partial^{2} v_{1}}{\partial x \partial t}\right)^{2}=-256 v_{1}^{2}\left|\Psi_{11}\left(\mu_{1}\right)\right|^{2}<0,
$$

viz., there is a saddle point, instead of a relative extremum.

It is worth noting that in order to obtain a similar result for $\mathrm{N}$ phase solutions with $N \geq 3$, the solution $p$ of the NLS equation must be considered as a simultaneous solution of $N$ evolutionary flows in the integrable NLS hierarchy of equations. Only two 
conditions define the critical points of the $x$ - and $t$-flows of the solution, and these two conditions are insufficient to imply a conclusion analogous to that of Eq. (19) on more than two Dirichlet eigenvalues.

\section{Invariant characteristic equation}

The characteristic equation of $\Psi$ is an invariant of both the $x$ flow and the $t$-flow of the solutions. It provides the integrals of motion necessary to integrate the differential equations. In particular, for the two-phase solutions, we seek the explicit construction of the $\mu_{1}$ and $\mu_{2}$ trajectories in terms of the symmetric polynomials of the roots of the invariant characteristic equation.

The characteristic equation of $\Psi$, viz., the invariant spectral curve, as defined by Eqs. (12), is

$\operatorname{det}(i w I-\Psi)=-w^{2}+\mathscr{R}(\lambda)=0$,

where

$\mathscr{R}(\lambda)=-\Psi_{11}^{2}-\Psi_{12} \Psi_{21}=\prod_{i=1}^{6}\left(\lambda-\lambda_{i}\right)$,

and it naturally defines a hyperelliptic Riemann surface of arithmetic genus two with points $P=(\lambda, w(\lambda)) \in \mathbb{C}^{2}$ lying on the complex algebraic curve $\mathscr{K}_{2}$ defined by Eq. (22). The curve $\mathscr{K}_{2}$ is a branched two-sheeted covering of the Riemann sphere with two points over the point at infinity; it is assumed that the curve is nonsingular, i.e., the branch points $\lambda_{i}$, for $i=1, \ldots, 6$, are distinct. Let the point $\infty^{+}$be defined as the point over infinity with $\lambda=\infty$ and $w(\lambda)=\lambda^{3}+O\left(\lambda^{2}\right)$, similarly $\infty^{-}$is the point over $\lambda=\infty$ with $w(\lambda)=-\lambda^{3}+O\left(\lambda^{2}\right)$. The curve $\mathscr{K}_{2}$ admits the usual hyperelliptic involution corresponding to sheet interchange,

$\iota: \mathscr{K}_{2} \rightarrow \mathscr{K}_{2}, \quad \iota(\lambda, w(\lambda))=(\lambda,-w(\lambda))$

and, because the coefficients of $\mathscr{R}(\lambda)$ are real, an anti-holomorphic involution

$*: \mathscr{K}_{2} \rightarrow \mathscr{K}_{2}, \quad *(\lambda, w(\lambda))=\left(\lambda^{*}, w(\lambda)^{*}\right)$,

where we use the same symbol $*$ for the involution acting on points of $\mathscr{K}_{2}$, as well as for complex conjugation of complex numbers. Note that the anti-holomorphic involution (25) leaves the sheets of the covering of the Riemann sphere unchanged, as can be seen by considering the action of $*$ on points in the vicinity of $P_{\infty}^{+}$, viz., if $\lambda \in \mathbb{R}$, then

$$
*(\lambda, w(\lambda))=\left(\lambda^{*}, w(\lambda)^{*}\right)=(\lambda, w(\lambda)) \text {. }
$$

The symmetry of the curve $\mathscr{K}_{2}$ expressed in the existence of the anti-holomorphic involution $*$ places reality conditions on the branch points of $\mathscr{K}_{2}$, the integrals of motion of the $x$ - and $t$-flows.

Corollary 1 (Real Curve). $\mathscr{K}_{2}$ must be a real algebraic curve, viz., the branch points are either real or come in complex-conjugate pairs.

The Dubrovin equations (17) can now be integrated in the standard way on the hyperelliptic Riemann surface [14].

Lemma 5 (Dubrovin Equations Reprised). Eq. (23) implies

$\Psi_{11}\left(\mu_{j}\right)=i \sqrt{\mathscr{R}\left(\mu_{j}\right)}$,

for $j=1,2$. Therefore, the motion of the Dirichlet eigenvalues is defined on $\mathscr{K}_{2}$ by the Dubrovin equations (17),

$$
\begin{aligned}
& \frac{\partial \mu_{1}}{\partial x}=2 i \frac{\sqrt{\mathscr{R}\left(\mu_{1}\right)}}{\mu_{1}-\mu_{2}}, \\
& \frac{\partial \mu_{2}}{\partial x}=2 i \frac{\sqrt{\mathscr{R}\left(\mu_{2}\right)}}{\mu_{2}-\mu_{1}}, \\
& \frac{\partial \mu_{1}}{\partial t}=-2\left(\mu_{2}+c_{2}\right) \frac{\partial \mu_{1}}{\partial x}, \\
& \frac{\partial \mu_{2}}{\partial t}=-2\left(\mu_{1}+c_{2}\right) \frac{\partial \mu_{2}}{\partial x} .
\end{aligned}
$$

Lemma 6. The $x$-flow and t-flow of $\mu_{1}$ and $\mu_{2}$ on $\mathscr{K}_{2}$ are linearized by the Abel mapping,

$\int_{\mu_{10}}^{\mu_{1}} \frac{d \mu_{1}}{\sqrt{\mathscr{R}\left(\mu_{1}\right)}}+\int_{\mu_{20}}^{\mu_{2}} \frac{d \mu_{2}}{\sqrt{\mathscr{R}\left(\mu_{2}\right)}}=4 i t$,

$\int_{\mu_{10}}^{\mu_{1}} \frac{\mu_{1} d \mu_{1}}{\sqrt{\mathscr{R}\left(\mu_{1}\right)}}+\int_{\mu_{20}}^{\mu_{2}} \frac{\mu_{2} d \mu_{2}}{\sqrt{\mathscr{R}\left(\mu_{2}\right)}}=2 i x-4 i c_{2} t$,

where $\mu_{10}$ and $\mu_{20}$ are constants of integration, viz., the initial values for $\mu_{1}$ and $\mu_{2}$.

It is important to remember that the values of $\mu_{10}$ and $\mu_{20}$ are not arbitrary but must satisfy the algebraic reality conditions imposed on the integrals of motion and, hence, on the loci of $\mu_{1}$ and $\mu_{2}$ by the characteristic equation (22). The explicit construction of the real loci of the two-phase Dirichlet eigenvalues does not appear in the earlier literature, but will be accomplished in the next section.

\section{Integrals of motion and the Dirichlet eigenvalues}

The symmetric polynomials of degree $i, \Sigma_{i}, i=1, \ldots, 4$, of $\mu_{1}, \mu_{2}, \mu_{1}^{*}, \mu_{2}^{*}$, must satisfy the following reality condition because the Dirichlet eigenvalues come in complex-conjugate pairs.

\section{Corollary 2 (Symmetric Polynomials of the Dirichlet Eigenvalues).}

$\Sigma_{1}, \Sigma_{2}, \Sigma_{3}, \Sigma_{4} \in \mathbb{R}$

and

$\Sigma_{4}=\left|\mu_{1} \mu_{2}\right|^{2} \geq 0$.

Moreover, because quartic polynomial equations have an explicit solution method, the algebraic constraints on the real loci of $\mu_{1}$ and $\mu_{2}$ can also be made explicit. Using the invariant spectral polynomial (22) and definition (23), the symmetric polynomials of degree $i, \Lambda_{i}, i=1, \ldots, 6$, of $\lambda_{i}, i=1, \ldots, 6$, the branch points of the invariant spectral curve, can be written in terms of the symmetric polynomials of degree $i, \Sigma_{i}, i=1, \ldots, 4$, of the Dirichlet eigenvalues $\mu_{1}, \mu_{2}, \mu_{1}^{*}, \mu_{2}^{*}$.

Lemma 7 (Symmetric Polynomials of Branch Points).

$\Lambda_{1}=-2 c_{2}$

$\Lambda_{2}=2 c_{1}+c_{2}^{2}$,

$\Lambda_{3}=-2 c_{1} c_{2}-2 c_{0}+\frac{1}{2} v_{2}+2 c_{2} v_{1}+v_{1} \Sigma_{1}$,

$\Lambda_{4}=-\frac{1}{2} c_{2} v_{2}-c_{2}^{2} v_{1}-c_{1} v_{1}+\frac{1}{4} v_{1}^{2}+2 c_{0} c_{2}$ $+c_{1}^{2}+v_{1} \Sigma_{2}$,

$\Lambda_{5}=-\frac{1}{4} v_{1} v_{2}-\frac{1}{2} c_{2} v_{1}^{2}+c_{0} v_{1}+\frac{1}{2} c_{1} v_{2}$

$-2 c_{0} c_{1}+c_{1} c_{2} v_{1}+v_{1} \Sigma_{3}$

$\Lambda_{6}=\frac{1}{4} c_{2} v_{1} v_{2}-c_{2} c_{0} v_{1}-\frac{1}{2} c_{0} v_{2}+\frac{1}{4} c_{2}^{2} v_{1}^{2}+c_{0}^{2}$

$+\frac{1}{16} v_{2}^{2}+v_{1} \Sigma_{4}$

Eq. (15) and the first three equations of (32) determine the three constants $c_{0}, c_{1}, c_{2}$, in terms of the branch points. 
Lemma 8 (Constants of Integration).

$c_{2}=-\frac{1}{2} \Lambda_{1}$

$c_{1}=\frac{1}{2} \Lambda_{2}-\frac{1}{8} \Lambda_{1}^{2}$,

$c_{0}=-\frac{1}{2} \Lambda_{3}+\frac{1}{4} \Lambda_{2} \Lambda_{1}-\frac{1}{16} \Lambda_{1}^{3}$.

Eq. (15) and the last three equations of (32) show that loci of the Dirichlet eigenvalues can be parametrized in terms of $v_{1}$ and $v_{2}$ by solving for $\Sigma_{i}, i=1, \ldots, 4$.

Lemma 9 (Symmetric Polynomials of Dirichlet Eigenvalues). If $v_{1}>$ 0 , then

$$
\begin{aligned}
\Sigma_{1}= & -\frac{1}{2} \frac{\nu_{2}}{v_{1}}+\Lambda_{1}, \\
\Sigma_{2}= & -\frac{1}{4} \Lambda_{1} \frac{\nu_{2}}{v_{1}}-\frac{1}{4} \nu_{1}+\frac{-\frac{5}{64} \Lambda_{1}^{4}+\frac{3}{8} \Lambda_{1}^{2} \Lambda_{2}-\frac{1}{4} \Lambda_{2}^{2}-\frac{1}{2} \Lambda_{1} \Lambda_{3}+\Lambda_{4}}{v_{1}} \\
& +\frac{1}{2} \Lambda_{2}+\frac{1}{8} \Lambda_{1}^{2}, \\
\Sigma_{3}= & \frac{1}{4} \nu_{2}+\left(\frac{1}{16} \Lambda_{1}^{2}-\frac{1}{4} \Lambda_{2}\right) \frac{\nu_{2}}{v_{1}}-\frac{1}{4} \Lambda_{1} \nu_{1} \\
& +\frac{\frac{1}{64} \Lambda_{1}^{5}-\frac{1}{8} \Lambda_{1}^{3} \Lambda_{2}+\frac{1}{8} \Lambda_{1}^{2} \Lambda_{3}+\frac{1}{4} \Lambda_{1} \Lambda_{2}^{2}-\frac{1}{2} \Lambda_{2} \Lambda_{3}+\Lambda_{5}}{v_{1}}+\frac{1}{2} \Lambda_{3}, \\
\Sigma_{4}= & \frac{1}{8} \Lambda_{1} \nu_{2}-\frac{1}{16} \frac{v_{2}^{2}}{v_{1}}-\frac{1}{16} \Lambda_{1}^{2} \nu_{1}+\left(-\frac{1}{32} \Lambda_{1}^{3}+\frac{1}{8} \Lambda_{1} \Lambda_{2}-\frac{1}{4} \Lambda_{3}\right) \frac{v_{2}}{v_{1}} \\
& +\frac{-\frac{1}{256} \Lambda_{1}^{6}+\frac{1}{32} \Lambda_{1}^{4} \Lambda_{2}-\frac{1}{16} \Lambda_{1}^{3} \Lambda_{3}-\frac{1}{16} \Lambda_{1}^{2} \Lambda_{2}^{2}+\frac{1}{4} \Lambda_{1} \Lambda_{2} \Lambda_{3}-\frac{1}{4} \Lambda_{3}^{2}+\Lambda_{6}}{v_{1}} \\
& +\frac{1}{32} \Lambda_{1}^{4}-\frac{1}{8} \Lambda_{1}^{2} \Lambda_{2}+\frac{1}{4} \Lambda_{1} \Lambda_{3} .
\end{aligned}
$$

Note that $\Lambda_{i} \in \mathbb{R}, i=1, \ldots, 6$, are real parameters and $v_{1}, v_{2} \in \mathbb{R}$ are real variables. Thus the four roots $\mu_{1}, \mu_{2}, \mu_{1}^{*}, \mu_{2}^{*} \in$ $\mathbb{C}$ of the quartic equation

$$
\begin{aligned}
\prod_{i=1}^{2}\left(\mu-\mu_{i}\right)\left(\mu-\mu_{i}^{*}\right) & =\mu^{4}-\Sigma_{1} \mu^{3}+\Sigma_{2} \mu^{2}-\Sigma_{3} \mu+\Sigma_{4} \\
& =0,
\end{aligned}
$$

each lie on a two-real-dimensional manifold parametrized by $v_{1}, v_{2} \in \mathbb{R}$. The explicit solutions for $\mu_{1}$ and $\mu_{2}$ can be found using the standard procedure for solving quartic equations. First the quartic polynomial in Eq. (35) is reduced by the transformation

$\mu=\hat{\mu}+\frac{1}{4} \Sigma_{1}$,

so that Eq. (35) becomes

$\hat{\mu}^{4}+\hat{\Sigma}_{2} \hat{\mu}^{2}-\hat{\Sigma}_{3} \hat{\mu}+\hat{\Sigma}_{4}=0$,

where

$\hat{\Sigma}_{2}=-\frac{3}{8} \Sigma_{1}^{2}+\Sigma_{2}$,

$\hat{\Sigma}_{3}=\frac{1}{8} \Sigma_{1}^{3}-\frac{1}{2} \Sigma_{1} \Sigma_{2}+\Sigma_{3}$,

$\hat{\Sigma}_{4}=-\frac{3}{256} \Sigma_{1}^{4}+\frac{1}{16} \Sigma_{1}^{2} \Sigma_{2}-\frac{1}{4} \Sigma_{1} \Sigma_{3}+\Sigma_{4}$.

Note that $\Sigma_{1} \in \mathbb{R}$, so the reality condition of Corollary 2 transfers unchanged to the shifted variables $\hat{\Sigma}_{2}, \hat{\Sigma}_{3}, \hat{\Sigma}_{4} \in \mathbb{R}$, and $\hat{\Sigma}_{4} \geq 0$, because the roots of Eq. (37) still come in two complex-conjugate pairs. Completing the square of the first two terms of Eq. (37) gives

$$
\left(\hat{\mu}^{2}+\frac{1}{2} \hat{\Sigma}_{2}\right)^{2}=\hat{\Sigma}_{3} \hat{\mu}+\frac{1}{4} \hat{\Sigma}_{2}^{2}-\hat{\Sigma}_{4}
$$

If $\hat{\Sigma}_{3}=0$, then Eq. (39) can be solved immediately to give

$$
\hat{\mu}=\frac{ \pm 1}{\sqrt{2}} \sqrt{-\hat{\Sigma}_{2}+s \sqrt{\hat{\Sigma}_{2}^{2}-4 \hat{\Sigma}_{4}}}
$$

where $s= \pm 1$. The reality condition of Theorem 1 implies that the shifted Dirichlet eigenvalues come in complex-conjugate pairs, so their values are constrained and the expressions for them in Eq. (40) can be simplified.

Corollary 3 (First Symmetric-Polynomial Constraint). Suppose $v_{1}>$ 0 . If $\hat{\Sigma}_{3}=0$, then the reality condition of Theorem 1 , viz., the Dirichlet eigenvalues come in complex-conjugate pairs, implies that $\hat{\Sigma}_{2} \geq$ $-2 \sqrt{\hat{\Sigma}_{4}}$, and the explicit expressions for the Dirichlet eigenvalues fall into two subcases.

1. If $\hat{\Sigma}_{2}^{2} \leq 4 \hat{\Sigma}_{4}$, then

$$
\begin{aligned}
& \hat{\mu}_{1}=-\frac{1}{2} \sqrt{-\hat{\Sigma}_{2}+2 \sqrt{\hat{\Sigma}_{4}}} \pm \frac{1}{2} i \sqrt{\hat{\Sigma}_{2}+2 \sqrt{\hat{\Sigma}_{4}}}, \\
& \hat{\mu}_{2}=\frac{1}{2} \sqrt{-\hat{\Sigma}_{2}+2 \sqrt{\hat{\Sigma}_{4}}} \pm \frac{1}{2} i \sqrt{\hat{\Sigma}_{2}+2 \sqrt{\hat{\Sigma}_{4}}} .
\end{aligned}
$$

2. If $\hat{\Sigma}_{2}>2 \sqrt{\hat{\Sigma}_{4}}$, then

$$
\begin{aligned}
& \hat{\mu}_{1}= \pm \frac{1}{\sqrt{2}} i \sqrt{\hat{\Sigma}_{2}+\sqrt{\hat{\Sigma}_{2}^{2}-4 \hat{\Sigma}_{4}}}, \\
& \hat{\mu}_{2}= \pm \frac{1}{\sqrt{2}} i \sqrt{\hat{\Sigma}_{2}-\sqrt{\hat{\Sigma}_{2}^{2}-4 \hat{\Sigma}_{4}}} .
\end{aligned}
$$

Proof. The result follows by direct calculation using Eq. (40).

If $\hat{\Sigma}_{3} \neq 0$, then a quantity $z$, to be determined, is added to the quantity in the parentheses on the left-hand side of Eq. (39),

$$
\left(\hat{\mu}^{2}+\frac{1}{2} \hat{\Sigma}_{2}+z\right)^{2}=2 z \hat{\mu}^{2}+\hat{\Sigma}_{3} \hat{\mu}+\frac{1}{4} \hat{\Sigma}_{2}^{2}-\hat{\Sigma}_{4}+\hat{\Sigma}_{2} z+z^{2}
$$

The quantity $z$ is chosen so that the right-hand side of the equation is a perfect square, which means that $z$ should be chosen so that the discriminant of the right-hand side, a quadratic polynomial in $\hat{\mu}$, is zero, viz.,

$z^{3}+\hat{\Sigma}_{2} z^{2}+\left(\frac{1}{4} \hat{\Sigma}_{2}^{2}-\hat{\Sigma}_{4}\right) z-\frac{1}{8} \hat{\Sigma}_{3}^{2}=0$

In fact the roots of Eq. (44) can be written down explicitly, if necessary.

Lemma 10. Suppose $v_{1}>0$. If $\hat{\Sigma}_{3} \neq 0$, then Eq. (44) has at least one positive root.

Proof. The existence of a positive root when $\hat{\Sigma}_{3} \neq 0$ follows immediately from the graph of the cubic polynomial in Eq. (44) with positive leading coefficient and negative constant coefficient. The explicit formula for the roots is the well-known Cardano formula, but it is not needed here.

Definition 1. Suppose $v_{1}>0$. If $\hat{\Sigma}_{3} \neq 0$, then $z$ is chosen, without loss of generality, to be a positive root of Eq. (44). 
Corollary 4 (Second Symmetric-Polynomial Constraint). Suppose $v_{1}>0$. If $\hat{\Sigma}_{3} \neq 0$, then the reality condition of Theorem 1 , viz., the Dirichlet eigenvalues come in complex-conjugate pairs, implies that

$\rho_{+}=\hat{\Sigma}_{2}+\frac{1}{\sqrt{2 z}} \hat{\Sigma}_{3}+z \geq 0$

$\rho_{-}=\hat{\Sigma}_{2}-\frac{1}{\sqrt{2 z}} \hat{\Sigma}_{3}+z \geq 0$

where $z>0$ is a root of Eq. (44). The explicit expressions for $\hat{\mu}_{1}=$ $\mu_{1}-\frac{1}{4} \Sigma_{1}$ and $\hat{\mu}_{2}=\mu_{2}-\frac{1}{4} \Sigma_{1}$ in terms of $v_{1}$ and $v_{2}$ are

$\hat{\mu}_{1}=-\sqrt{\frac{z}{2}} \pm \frac{1}{\sqrt{2}} i \sqrt{\hat{\Sigma}_{2}+\frac{1}{\sqrt{2 z}} \hat{\Sigma}_{3}+z}$

$\hat{\mu}_{2}=\sqrt{\frac{z}{2}} \pm \frac{1}{\sqrt{2}} i \sqrt{\hat{\Sigma}_{2}-\frac{1}{\sqrt{2 z}} \hat{\Sigma}_{3}+z}$.

In particular, if $\hat{\Sigma}_{3} \neq 0$, then $\mu_{1} \neq \mu_{2}$.

Proof. Since $z>0$ is a root of the cubic equation (44), the righthand side of Eq. (43) is a perfect square,

$$
\left(\hat{\mu}^{2}+\frac{1}{2} \hat{\Sigma}_{2}+z\right)^{2}=2 z\left(\hat{\mu}+\frac{1}{4 z} \hat{\Sigma}_{3}\right)^{2} \text {. }
$$

Therefore

$\hat{\mu}^{2}+s \sqrt{2 z} \hat{\mu}+\frac{1}{2} \hat{\Sigma}_{2}+s \frac{1}{2 \sqrt{2 z}} \hat{\Sigma}_{3}+z=0$,

where $s= \pm 1$. Finally, solving the quadratic equation (48) gives explicit expressions for the shifted Dirichlet eigenvalues, together with the algebraic constraints that are equivalent to the reality condition. The reality constraint of Corollary 3 represents the limiting case in which $\hat{\Sigma}_{3} \rightarrow 0$, since the Dirichlet eigenvalues are continuous functions of the symmetric polynomials of the branch points.

Theorem 3 (Boundary of the Region Satisfying the Reality Condition). Suppose that $v_{1}>0$. Then the boundary of the set of points $\left(v_{1}, v_{2}\right)$ for which the reality condition of Theorem 1 is satisfied, viz., the Dirichlet eigenvalues come in complex-conjugate pairs, is a subset of an algebraic set defined by

$16 \hat{\Sigma}_{4}\left(\hat{\Sigma}_{2}^{2}-4 \hat{\Sigma}_{4}\right)^{2}-\hat{\Sigma}_{3}^{2}\left(4 \hat{\Sigma}_{2}^{3}-144 \hat{\Sigma}_{2} \hat{\Sigma}_{4}+27 \hat{\Sigma}_{3}^{2}\right)=0$.

On this boundary, if $\hat{\Sigma}_{3} \neq 0$, then the root $z$ is given by

$z=-\frac{1}{3} \hat{\Sigma}_{2}+\frac{1}{3} \sqrt{\hat{\Sigma}_{2}^{2}+12 \hat{\Sigma}_{4}}>0$.

Proof. The Dirichlet eigenvalues are either real or come in complex-conjugate pairs, and they are continuous functions of the real symmetric polynomials. Therefore, the number of complexconjugate pairs of Dirichlet eigenvalues can change only when $\rho_{+}=0$ or $\rho_{-}=0$, if $\hat{\Sigma}_{3} \neq 0$, or when $\rho_{+} \rightarrow 0$ or $\rho_{-} \rightarrow 0$ as $\hat{\Sigma}_{3} \rightarrow 0$, by the algebraic constraints of Corollaries 3 and 4 . Since points where $\hat{\Sigma}_{3}=0$ are limit points of points where $\hat{\Sigma}_{3} \neq 0$, it is sufficient to derive the boundary set with the assumption that $\hat{\Sigma}_{3} \neq 0$.

When $\hat{\Sigma}_{3} \neq 0, z>0$, so the set of $\left(v_{1}, v_{2}\right)$ for which $\hat{\Sigma}_{3} \neq 0$ and either $\rho_{+}=0$ or $\rho_{-}=0$ is equivalent to the set of solutions of

$2 z\left(z+\hat{\Sigma}_{2}\right)^{2}-\hat{\Sigma}_{3}^{2}=0$.
Now $z>0$ is also a root of the cubic equation (44). The set of points $\left(v_{1}, v_{2}\right)$ for which Eqs. (44) and (51) have a common root is defined by setting the resultant of the two equations to zero. The resultant of Eqs. (44) and (51) is a nonzero constant multiple of

$\hat{\Sigma}_{3}^{2}\left(16 \hat{\Sigma}_{4}\left(\hat{\Sigma}_{2}^{2}-4 \hat{\Sigma}_{4}\right)^{2}-\hat{\Sigma}_{3}^{2}\left(4 \hat{\Sigma}_{2}^{3}-144 \hat{\Sigma}_{2} \hat{\Sigma}_{4}+27 \hat{\Sigma}_{3}^{2}\right)\right)$.

Since $\hat{\Sigma}_{3} \neq 0$, Eq. (49) follows immediately.

The expression for the common root in Eq. (50) follows by reducing the degree of $z$ in the simultaneous Eqs. (44) and (51). Note that if $\hat{\Sigma}_{4}=0$ and $\hat{\Sigma}_{3} \neq 0$, then Eq. (49) implies that $\hat{\Sigma}_{2}<0$, so that $z$ given by Eq. (50) is, in fact, always positive when $\hat{\Sigma}_{3} \neq 0$.

\section{Dependence of extrema on the branch points}

The effective integration of the $\mu_{1}$ and $\mu_{2}$ equations can be accomplished by transforming the evolution Eqs. (18) for $v_{1}$ and $\nu_{2}$, using the explicit expressions for the shifted variables $\hat{\mu}_{1}$ and $\hat{\mu}_{2}$. In the case that $v_{1}>0$ and $\hat{\Sigma}_{3} \neq 0$, Corollary 4 implies that

$$
\begin{aligned}
\frac{\partial v_{1}}{\partial x}= & -2 \sqrt{2} v_{1}\left(\sqrt{\rho_{+}}+\sqrt{\rho_{-}}\right) \\
\frac{\partial v_{2}}{\partial x}= & 4 v_{1} \sqrt{z}\left(\sqrt{\rho_{+}}-\sqrt{\rho_{-}}\right) \\
& +\sqrt{2} v_{1}\left(\Sigma_{1}+4 c_{2}\right)\left(\sqrt{\rho_{+}}+\sqrt{\rho_{-}}\right), \\
\frac{\partial v_{1}}{\partial t}= & \frac{\partial v_{2}}{\partial x} \\
\frac{\partial v_{2}}{\partial t}= & -4 \sqrt{2} v_{1}\left(\left(z+\rho_{-}\right) \sqrt{\rho_{+}}+\left(z+\rho_{+}\right) \sqrt{\rho_{-}}\right) \\
& +\sqrt{2} v_{1}\left(-\frac{1}{2} \Sigma_{1}^{2}-4 c_{2} \Sigma_{1}-8 c_{2}^{2}\right)\left(\sqrt{\rho_{+}}+\sqrt{\rho_{-}}\right) \\
& -4 v_{1} \sqrt{z}\left(\Sigma_{1}+4 c_{2}\right)\left(\sqrt{\rho_{+}}-\sqrt{\rho_{-}}\right) .
\end{aligned}
$$

Moreover, if an oscillatory smooth bounded two-phase solution exists, there must be at least one positive relative extremum for $v_{1}$, which can occur only if $\mu_{1}=\mu_{1}^{*}$ and $\mu_{2}=\mu_{2}^{*}$, provided $\mu_{1} \neq \mu_{2}$. It is now possible to characterize explicitly all choices of the branch points of $\mathscr{K}_{2}$ for which the solution for $v_{1}$ could possibly oscillate on an interval of non-negative values.

Lemma 11. Suppose $v_{1}>0$. Then $\mu_{1}=\mu_{1}^{*}$ and $\mu_{2}=\mu_{2}^{*}$ if and only if $\hat{\Sigma}_{3}=0$ and $\hat{\Sigma}_{2}=-2 \sqrt{\hat{\Sigma}_{4}}$. Also, $\mu_{1}=\mu_{2}^{*}$ or $\mu_{1}=\mu_{2}$ if and only if $\hat{\Sigma}_{3}=0$ and $\hat{\Sigma}_{2}=2 \sqrt{\hat{\Sigma}_{4}}$.

Proof. Corollaries 3 and 4, containing the explicit expressions in Eqs. (41), (42), and (46), imply the stated results.

Lemma 12. If $p(x, t)$ is a smooth bounded two-phase solution of the NLS equation (1) and $v_{1}(x, t)=|p(x, t)|^{2}$, then the nonzero extrema of $v_{1}(x, t)$ must occur at points $\left(v_{1}, v_{2}\right)$ where $\hat{\Sigma}_{3}=0$ and $\hat{\Sigma}_{2}^{2}-4 \hat{\Sigma}_{4}=0$.

Proof. Theorem 2 shows that extrema can only occur when either (i) $\mu_{1}=\mu_{2}$ or (ii) $\mu_{1}=\mu_{1}^{*}$ and $\mu_{2}=\mu_{2}^{*}$. Together with the previous lemma, the result follows. Alternatively, the same result can be obtained from the system of Eqs. (53) and the explicit expressions in Corollaries 3 and 4 for the Dirichlet eigenvalues.

Lemma 13. Suppose $v_{1}>0$. Then $\hat{\Sigma}_{3}=0$ and $\hat{\Sigma}_{2}^{2}-4 \hat{\Sigma}_{4}=0$ if and only if $P\left(v_{1}\right)=0$, where $P\left(v_{1}\right)$ is a polynomial of degree ten in $v_{1}$.

Proof. $P\left(v_{1}\right)$ is the resultant in the variable $v_{2}$ of the two polynomials of $\nu_{2}$ given by $\nu_{1}^{3} \hat{\Sigma}_{3}$ and $v_{1}^{4}\left(\hat{\Sigma}_{2}^{2}-4 \hat{\Sigma}_{4}\right)$. 
The effectiveness of the integration method presented in this paper lies in the fact, stated in the following theorem, that the roots of the polynomial equation $P\left(v_{1}\right)=0$ can be expressed explicitly in terms of the branch points of the Riemann surface $\mathscr{K}_{2}$. In the following, the resultant polynomial $P\left(v_{1}\right)$ is assumed to be defined uniquely up to a nonzero constant multiplicative factor.

Theorem 4. If $p(x, t)$ is a smooth bounded two-phase solution of the NLS equation ( 1$)$, then $v_{1}(x, t)$ oscillates on an interval bounded either by zero or by positive roots of the polynomial equation $P\left(v_{1}\right)=$ 0 given in Lemma 13. The polynomial $P\left(v_{1}\right)$ is also a resolvent polynomial for the factorization of the reduced form of $\mathscr{R}(\lambda)$ into two cubic factors. In particular, the ten roots of $P\left(v_{1}\right)=0$, together with the corresponding $v_{2}$ values, are given explicitly in terms of the six branch points $\lambda_{i}, i=1, \ldots, 6$, of $\mathscr{K}_{2}$ by the formulas

$v_{1}=-\frac{1}{4} \chi_{1}^{2}$

$v_{2}=-\frac{1}{2} \chi_{1} \chi_{2}$,

where

$\chi_{1}=\lambda_{\pi(1)}+\lambda_{\pi(2)}+\lambda_{\pi(3)}-\lambda_{\pi(4)}-\lambda_{\pi(5)}-\lambda_{\pi(6)}$,

$\chi_{2}=\lambda_{\pi(1)}^{2}+\lambda_{\pi(2)}^{2}+\lambda_{\pi(3)}^{2}-\lambda_{\pi(4)}^{2}-\lambda_{\pi(5)}^{2}-\lambda_{\pi(6)}^{2}$,

and $(\pi(1), \pi(2), \pi(3), \pi(4), \pi(5), \pi(6)) \in S_{6}$, the permutation group of order six.

Proof. Lemmas 11 and 12 show that nonzero extrema of $v_{1}$ occur only if $P\left(v_{1}\right)=0$. When $v_{1}>0, P\left(v_{1}\right)=0$ if and only if (i) $\mu_{1}=\mu_{2}$ or (ii) $\mu_{1}=\mu_{1}^{*}$ and $\mu_{2}=\mu_{2}^{*}$ or (iii) $\mu_{1}=\mu_{2}^{*}$. In each of these cases, i.e., for each of the ten roots of $P\left(v_{1}\right)=0$, the sextic $\mathscr{R}$ factors into the product of two cubics $Q_{1}$ and $Q_{2}$. Specifically, in the case $\mu_{1}=\mu_{1}^{*}$ and $\mu_{2}=\mu_{2}^{*}$ or the case $\mu_{1}=\mu_{2}^{*}$,

$$
\begin{aligned}
\mathscr{R}(\lambda) & =-\Psi_{11}(\lambda)^{2}+v_{1}\left(\lambda-\mu_{1}\right)^{2}\left(\lambda-\mu_{2}\right)^{2}, \\
& =Q_{1}(\lambda) Q_{2}(\lambda),
\end{aligned}
$$

where

$Q_{1}=i \Psi_{11}(\lambda)+i \sqrt{\nu_{1}}\left(\lambda-\mu_{1}\right)\left(\lambda-\mu_{2}\right)$,

$Q_{2}=i \Psi_{11}(\lambda)-i \sqrt{\nu_{1}}\left(\lambda-\mu_{1}\right)\left(\lambda-\mu_{2}\right)$.

There is a similar factorization in the case $\mu_{1}=\mu_{2}$. The zeros of each cubic factor form two non-overlapping sets, each comprised of three distinct branch points. Using Vieta's formulas to express the coefficients of the cubic factors $Q_{1}$ and $Q_{2}$ in terms of the three roots of $Q_{1}=0$ and the three roots of $Q_{2}=0$, explicitly solving for $v_{1}$ and $v_{2}$ from the coefficients of the quadratic and constant terms of $Q_{1}$ and $Q_{2}$, and eliminating the terms depending on $\mu_{1}$ and $\mu_{2}$, leads to the stated formulas for $v_{1}$ and $v_{2}$. Notice that both $v_{1}$ and $v_{2}$ are invariant under the actions of re-ordering the first three or the last three summands, and also swapping the first three summands with the second three summands, so that the number of values of either $v_{1}$ or $v_{2}$ is $6 ! /(3 ! 3 ! 2 !)=10$, as expected.

In fact, explicit calculation shows that if $v_{1}=-a_{2}^{2}$, then the degree ten polynomial $P\left(v_{1}\right)$ is the resolvent polynomial for the factorization into two cubic factors, one of the factors being $\lambda^{3}+$ $a_{2} \lambda^{2}+a_{1} \lambda+a_{0}$, of the reduced sextic polynomial of the sextic polynomial $\mathscr{R}(\lambda)$. The resolvent polynomial can be calculated by successive eliminations of variables from the equations obtained by equating coefficients of the expanded product of the desired factors equal to the polynomial to be factored [31].

Theorem 5. If a smooth bounded two-phase solution of the NLS equation (1) exists, then the values of $v_{1}$ and $v_{2}$ at critical points of $v_{1}(x, t)$ are given by

$v_{1}=-\frac{1}{4} \chi_{1}^{2}$

$v_{2}=-\frac{1}{2} \chi_{1} \chi_{2}$

where

$\chi_{1}=\lambda_{\pi(1)}+\lambda_{\pi(2)}+\lambda_{\pi(3)}-\lambda_{\pi(4)}-\lambda_{\pi(5)}-\lambda_{\pi(6)}$,

$\chi_{2}=\lambda_{\pi(1)}^{2}+\lambda_{\pi(2)}^{2}+\lambda_{\pi(3)}^{2}-\lambda_{\pi(4)}^{2}-\lambda_{\pi(5)}^{2}-\lambda_{\pi(6)}^{2}$.

Moreover, if $\mu_{1} \neq \mu_{2}$ at a critical point, then the following expressions of the second-order partial derivatives at the critical point have a similarly explicit dependence on the branch points,

$$
\begin{aligned}
v_{1 x x} v_{1 t t}-v_{1 x t}^{2}= & 256 v_{1}^{2} \Psi_{11}\left(\mu_{1}\right) \Psi_{11}\left(\mu_{2}\right) \\
= & -16 \chi_{1} \prod_{k=1}^{3}\left(\lambda_{\pi(k)}-\lambda_{\pi(4)}\right)\left(\lambda_{\pi(k)}-\lambda_{\pi(5)}\right) \\
& \times\left(\lambda_{\pi(k)}-\lambda_{\pi(6)}\right),
\end{aligned}
$$

and

$$
\begin{aligned}
v_{1 x x}= & 8 i v_{1} \frac{\Psi_{11}\left(\mu_{1}\right)-\Psi_{11}\left(\mu_{2}\right)}{\mu_{1}-\mu_{2}} \\
= & -2\left(\lambda_{\pi(1)}-\lambda_{\pi(4)}\right)\left(\lambda_{\pi(1)}-\lambda_{\pi(5)}\right)\left(\lambda_{\pi(2)}-\lambda_{\pi(4)}\right) \\
& \times\left(\lambda_{\pi(2)}-\lambda_{\pi(5)}\right)-2\left(\lambda_{\pi(1)}-\lambda_{\pi(4)}\right)\left(\lambda_{\pi(1)}-\lambda_{\pi(6)}\right) \\
& \times\left(\lambda_{\pi(3)}-\lambda_{\pi(4)}\right)\left(\lambda_{\pi(3)}-\lambda_{\pi(6)}\right)-2\left(\lambda_{\pi(2)}-\lambda_{\pi(5)}\right) \\
& \times\left(\lambda_{\pi(2)}-\lambda_{\pi(6)}\right)\left(\lambda_{\pi(3)}-\lambda_{\pi(5)}\right)\left(\lambda_{\pi(3)}-\lambda_{\pi(6)}\right) \\
& -2 \chi_{1}\left(\lambda_{\pi(1)}-\lambda_{\pi(4)}\right)\left(\lambda_{\pi(2)}-\lambda_{\pi(5)}\right)\left(\lambda_{\pi(3)}-\lambda_{\pi(6)}\right),
\end{aligned}
$$

where $(\pi(1), \pi(2), \pi(3), \pi(4), \pi(5), \pi(6)) \in S_{6}$, where $S_{6}$ is the permutation group of order 6 .

Proof. The first part of the theorem is just a restatement of the result of the previous theorem, viz., by Lemma 4, at a critical point of $v_{1}(x, t)$ either (i) $\mu_{1}=\mu_{1}^{*}$ and $\mu_{2}=\mu_{2}^{*}$ or (ii) $\mu_{1}=$ $\mu_{2}^{*}$. In either case $P\left(v_{1}\right)=0$ by Lemmas 10 and 12 . According to Theorem $4, v_{1}$ and $v_{2}$ are given by the stated formulas for one of the permutations of the indices. The expressions for the second-order partial derivatives at the critical point, assuming $\mu_{1} \neq \mu_{2}$, follow by direct substitution of (i) $\mu_{1}=\mu_{1}^{*}$ and $\mu_{2}=\mu_{2}^{*}$ or (ii) $\mu_{1}=\mu_{2}^{*}$ into the expressions for the secondorder partial derivatives, resulting in formulas in terms of $\Psi_{11}(\mu)$. Then the explicit expressions for $\mu_{1}$ and $\mu_{2}$ in terms of $v_{1}$ and $v_{2}$ are substituted into $\Psi_{11}(\mu)$, using Eqs. (36) and (41). Finally the explicit expressions for $v_{1}$ and $v_{2}$ in terms of the branch points, given by Eq. (58), are used.

\section{Branch point reality conditions}

Using Theorem 4, the reality condition of Corollary 1 on the nonsingular algebraic curve $\mathscr{K}_{2}$ can be strengthened.

Lemma 14. If $\lambda_{1}, \lambda_{2}, \lambda_{3}, \lambda_{4}, \lambda_{5}, \lambda_{6} \in \mathbb{R}$, then $P\left(v_{1}\right)=0$ has no positive roots.

Proof. The result follows immediately from Eq. (54).

Lemma 15. If $v_{2} \in \mathbb{R}, \lambda_{1}=\lambda_{2}^{*}$, and $\lambda_{3}, \lambda_{4}, \lambda_{5}, \lambda_{6} \in \mathbb{R}$, then $P\left(v_{1}\right)=0$ has no positive roots.

Proof. The proof is by contradiction. If $P\left(v_{1}\right)=0$ and $v_{1}>0$, then Eq. (54) implies, for example, that $\lambda_{3}+\lambda_{4}=\lambda_{5}+\lambda_{6}$ and, also, that $\lambda_{3} \lambda_{4}=\lambda_{5} \lambda_{6}$, since $\nu_{2}$ is real. However these two equalities imply that $\lambda_{3}$ is equal to at least one of $\lambda_{5}$ or $\lambda_{6}$, contradicting the distinctness of the branch points, viz., the nonsingularity assumption on $\mathscr{K}_{2}$. 
Lemma 16. If $v_{2} \in \mathbb{R}, \lambda_{1}=\lambda_{2}^{*}, \lambda_{3}=\lambda_{4}^{*}, \lambda_{5}, \lambda_{6} \in \mathbb{R}$, then $P\left(v_{1}\right)=0$ has no positive roots.

Proof. The proof of this lemma is similar to the previous lemma. If $v_{1}>0$ in Eq. (54) and the corresponding $v_{2} \in \mathbb{R}$ in Eq. (54), then either $\lambda_{5}=\lambda_{6}$ or, otherwise, $\lambda_{1}$ equals one of $\lambda_{5}$ or $\lambda_{6}$.

Theorem 6 (Reality Conditions for Bounded Two-Phase Solutions). If the NLS equation (1) has a smooth bounded two-phase solution, and $\mathscr{K}_{2}$ is non-singular, i.e., its six branch points are distinct, then the branch points form three complex-conjugate pairs. $P\left(v_{1}\right)=0$ has exactly four distinct non-negative roots, these are the possible nonzero bounds of the two-phase solution, and the corresponding values of $\nu_{2}$ given by Eq. (54) are also real. In particular, if $\lambda_{1}=\lambda_{2}^{*}=$ $r_{1}+i s_{1}, \lambda_{3}=\lambda_{4}^{*}=r_{2}+i s_{2}$ and $\lambda_{5}=\lambda_{6}^{*}=r_{3}+i s_{3}$, with $0<s_{1} \leq s_{2} \leq s_{3}$, then the four real roots of $P\left(v_{1}\right)=0$ are

$0 \leq v_{1}^{(1)}<v_{1}^{(2)}<v_{1}^{(3)}<v_{1}^{(4)}$,

where

$v_{1}^{(1)}=\left(s_{1}+s_{2}-s_{3}\right)^{2}$,

$v_{1}^{(2)}=\left(s_{1}-s_{2}+s_{3}\right)^{2}$,

$v_{1}^{(3)}=\left(-s_{1}+s_{2}+s_{3}\right)^{2}$,

$v_{1}^{(4)}=\left(s_{1}+s_{2}+s_{3}\right)^{2}$,

and

$v_{2}^{(1)}=4\left(s_{1}+s_{2}-s_{3}\right)\left(r_{1} s_{1}+r_{2} s_{2}-r_{3} s_{3}\right)$,

$v_{2}^{(2)}=4\left(s_{1}-s_{2}+s_{3}\right)\left(r_{1} s_{1}-r_{2} s_{2}+r_{3} s_{3}\right)$,

$v_{2}^{(3)}=4\left(-s_{1}+s_{2}+s_{3}\right)\left(-r_{1} s_{1}+r_{2} s_{2}+r_{3} s_{3}\right)$,

$v_{2}^{(4)}=4\left(s_{1}+s_{2}+s_{3}\right)\left(r_{1} s_{1}+r_{2} s_{2}+r_{3} s_{3}\right)$.

Proof. The preceding lemmas eliminate any other possibilities for branch points satisfying the reality conditions. Theorem 4, Eq. (54), provides the explicit expressions for the four real roots. The explicit expressions for $v_{1}$ show that the four real values are distinct. Otherwise at least one of $s_{1}, s_{2}$, or $s_{3}$ would be zero, contradicting the assumption that the branch points are distinct. Notice that the set of four values $v_{1}^{(i)}, i=1, \ldots, 4$, is invariant with respect to labeling the branch points, but the specific ordering of the four values, with the exception of the largest value, depends on the choice of labeling of the imaginary parts of the branch points. In particular, with the labeling $0<s_{1} \leq s_{2} \leq s_{3}$, the ordering $0 \leq v_{1}^{(1)}<v_{1}^{(2)}<v_{1}^{(3)}<v_{1}^{(4)}$ follows immediately by simple algebra.

\section{Discussion of the labeling of the branch points.}

Without loss of generality, the labeling of the three real parts of the branch points can be chosen so that $r_{1} \leq r_{2} \leq r_{3}$, or the labeling of the absolute values of the imaginary parts of the branch points can be chosen so that $0<s_{1} \leq s_{2} \leq s_{3}$. However, these two conventions are not, in general, simultaneously valid. In this manuscript both conventions will be used in different contexts.

1. In Theorem 6 it is convenient to know the ordering of the imaginary parts of the branch points, because this determines the relative order of $v_{1}^{(i)}, i=1, \ldots, 4$. In the case of the one-phase solutions, the corresponding formulas can be obtained as a limiting case, for example, by choosing a labeling order for the imaginary parts, viz., $0<s_{1} \leq s_{2} \leq s_{3}$, and allowing the smallest imaginary part to approach zero. In this case the maximum squared modulus is $\left(s_{2}+s_{3}\right)^{2}$ and the minimum squared modulus is $\left(s_{3}-s_{2}\right)^{2}$. These two formulas are symmetric with respect to interchanging $s_{2}$ and $s_{3}$. In the two-phase case the set of four values $v_{1}^{(i)}, i=1, \ldots, 4$, is also symmetric with respect to changing the labeling of the branch points, but the ordering of these four values is no longer invariant, except for the maximum value $v_{1}^{(4)}$.

2. In the Appendix and the solution of the Jacobi inversion problem, a specific set of canonical cycles on the Riemann surface must be chosen, as in Fig. 7. In this case, it is convenient to label the branch points so that $r_{1} \leq r_{2} \leq r_{3}$. In the integration of the solution, the initial conditions will be placed at $\left(v_{1}^{(4)}, v_{2}^{(4)}\right)$, using the corresponding formulas for $\mu_{1}$ and $\mu_{2}$ given in Lemma 18. Since these formulas are symmetric with respect to labeling of the branch points, they are equally valid for either labeling convention of the branch points.

Lemma 17. If a two-phase solution of the NLS equation (1) exists, then it is bounded, viz., there exists a positive number $M \in \mathbb{R}$ such that $v_{1}(x, t)^{2}+v_{2}(x, t)^{2}<M$ for all $x$ and $t$.

Proof. First we show that $v_{1}$ is bounded.

1. If $v_{1} \rightarrow \infty$ and $v_{2}=o\left(v_{1}\right)$ and $\Lambda_{1} \neq 0$, then $\Sigma_{4} \sim-\frac{1}{16} \Lambda_{1}^{2} v_{1}<$ 0 , which is impossible since $\Sigma_{4} \geq 0$.

2. If $v_{1} \rightarrow \infty$ and $v_{2}=o\left(v_{1}\right)$ and $\Lambda_{1}=0$, then $\Sigma_{4} \sim-\frac{v_{2}^{2}}{16 v_{1}}<0$, also impossible.

3. If $v_{1} \rightarrow \infty$ and $v_{2}=O\left(v_{1}\right)$, then $\hat{\Sigma}_{2} \sim-\frac{1}{4} v_{1}, \hat{\Sigma}_{3} \sim-\frac{1}{8} \Lambda_{1} v_{1}+$ $\frac{3}{16} v_{2}$, and $z \rightarrow 0$. Hence

$\hat{\Sigma}_{2} \pm \frac{\hat{\Sigma}_{3}}{\sqrt{2 z}}+z \sim-\frac{v_{1}}{4}$ or $\pm \frac{-\frac{1}{8} \Lambda_{1} v_{1}+\frac{3}{16} v_{2}}{\sqrt{2 z}}$,

so that, in any case, at least one of $\rho_{1}$ or $\rho_{2}$ will become negative, which contradicts the reality condition of Corollary 4.

4. If $v_{1} \rightarrow \infty$ and $\frac{v_{2}}{v_{1}} \rightarrow \infty$, then $\Sigma_{4} \sim-\frac{v_{2}^{2}}{v_{1}}<0$, which is also impossible.

The preceding cases are exhaustive and show that $v_{1}$ is bounded. Consequently, if $\left|v_{2}\right| \rightarrow \infty$, then

$\Sigma_{4} \sim-\frac{1}{16} \frac{v_{2}^{2}}{v_{1}}$,

which contradicts $\Sigma_{4} \geq 0$. Hence $\left|v_{2}\right|$ is also bounded.

Lemma 18. Suppose $\lambda_{1}=\lambda_{2}^{*}=r_{1}+i s_{1}, \lambda_{3}=\lambda_{4}^{*}=r_{2}+i s_{2}$ and $\lambda_{5}=\lambda_{6}^{*}=r_{3}+i s_{3}$, with $s_{1}, s_{2}, s_{3}>0$. If a smooth twophase solution of the NLS equation (1) exists in a neighborhood of a point where $v_{1}(x, t)=v_{1}^{(4)}$, then $v_{1}=v_{1}^{(4)}>0$ is a relative maximum of $v_{1}(x, t)$. Moreover, the values of the corresponding solutions $\left(\mu_{1}, \sigma_{1} \sqrt{\mathscr{R}\left(\mu_{1}\right)}\right) \in \mathbb{R}^{2}$ and $\left(\mu_{2}, \sigma_{2} \sqrt{\mathscr{R}\left(\mu_{2}\right)}\right) \in \mathbb{R}^{2}$ of the Dubrovin equations on the Riemann surface $\mathscr{K}_{2}$ at this point are real and are given by explicit formulas,

$\mu_{1}=\frac{r_{1}\left(s_{2}+s_{3}\right)+r_{2}\left(s_{1}+s_{3}\right)+r_{3}\left(s_{1}+s_{2}\right)-\sqrt{A(\vec{r}, \vec{s})}}{2\left(s_{1}+s_{2}+s_{3}\right)}$,

$\mu_{2}=\frac{r_{1}\left(s_{2}+s_{3}\right)+r_{2}\left(s_{1}+s_{3}\right)+r_{3}\left(s_{1}+s_{2}\right)+\sqrt{A(\vec{r}, \vec{s})}}{2\left(s_{1}+s_{2}+s_{3}\right)}$,

where

$$
\begin{aligned}
A(\vec{r}, \vec{s})= & 4 s_{1} s_{2} s_{3}\left(s_{1}+s_{2}+s_{3}\right)+s_{1}^{2}\left(r_{2}-r_{3}\right)^{2} \\
& +s_{2}^{2}\left(r_{1}-r_{3}\right)^{2}+s_{3}^{2}\left(r_{1}-r_{2}\right)^{2}+2 s_{1} s_{3}\left(r_{2}-r_{3}\right) \\
& \times\left(r_{2}-r_{1}\right)+2 s_{2} s_{3}\left(r_{1}-r_{3}\right)\left(r_{1}-r_{2}\right) \\
& +2 s_{1} s_{2}\left(r_{3}-r_{1}\right)\left(r_{3}-r_{2}\right),
\end{aligned}
$$

and $A(\vec{r}, \vec{s})>0$. The hyperelliptic irrationalities are given by

$\sigma_{i} \sqrt{\mathscr{R}\left(\mu_{i}\right)}=\sigma_{i}\left|\mu_{i}-\lambda_{1}\right|\left|\mu_{i}-\lambda_{3}\right|\left|\mu_{i}-\lambda_{5}\right|$,

for $i=1,2$, with the hyperelliptic sheets given by $\sigma_{1}=-1$ and $\sigma_{2}=1$. 
Proof. Direct substitution shows that when $\left(v_{1}, v_{2}\right)=\left(v_{1}^{(4)}, v_{2}^{(4)}\right)$,

$\hat{\Sigma}_{2}=-\frac{A(\vec{r}, \vec{s})}{2\left(s_{1}+s_{2}+s_{3}\right)^{2}}<0$

where

$$
\begin{aligned}
A(\vec{r}, \vec{s})= & 4 s_{1} s_{2} s_{3}\left(s_{1}+s_{2}+s_{3}\right)+s_{1}^{2}\left(r_{2}-r_{3}\right)^{2}+s_{2}^{2}\left(r_{1}-r_{3}\right)^{2} \\
& +s_{3}^{2}\left(r_{1}-r_{2}\right)^{2}+2 s_{1} s_{3}\left(r_{2}-r_{3}\right)\left(r_{2}-r_{1}\right) \\
& +2 s_{2} s_{3}\left(r_{1}-r_{3}\right)\left(r_{1}-r_{2}\right)+2 s_{1} s_{2}\left(r_{3}-r_{1}\right)\left(r_{3}-r_{2}\right) \\
= & 4 s_{1} s_{2} s_{3}\left(s_{1}+s_{2}+s_{3}\right)+\left(s_{1}\left(r_{2}-r_{3}\right)+s_{3}\left(r_{1}-r_{2}\right)\right)^{2} \\
& +s_{2}^{2}\left(r_{1}-r_{3}\right)^{2}+2 s_{2} s_{3}\left(r_{1}-r_{3}\right)\left(r_{1}-r_{2}\right) \\
& +2 s_{1} s_{2}\left(r_{3}-r_{1}\right)\left(r_{3}-r_{2}\right) \\
> & 0 .
\end{aligned}
$$

The positivity of $A(\vec{r}, \vec{s})$ follows from the fact that the first expression in Eq. (70) for $A(\vec{r}, \vec{s})$ is symmetric with respect to interchange of the indices $i=1,2,3$. In particular, if $s_{1}, s_{2}, s_{3}>0$ and $r_{1} \leq r_{2} \leq r_{3}$, then the second expression for $A$ is positive. Thus $\hat{\Sigma}_{2}=-2 \sqrt{\hat{\Sigma}_{4}}$ and, hence, $\mu_{1}=\mu_{1}^{*}$ and $\mu_{2}=\mu_{2}^{*}$, with

$\mu_{1}=\frac{r_{1}\left(s_{2}+s_{3}\right)+r_{2}\left(s_{1}+s_{3}\right)+r_{3}\left(s_{1}+s_{2}\right)-\sqrt{A(\vec{r}, \vec{s})}}{2\left(s_{1}+s_{2}+s_{3}\right)}$,

$\mu_{2}=\frac{r_{1}\left(s_{2}+s_{3}\right)+r_{2}\left(s_{1}+s_{3}\right)+r_{3}\left(s_{1}+s_{2}\right)+\sqrt{A(\vec{r}, \vec{s})}}{2\left(s_{1}+s_{2}+s_{3}\right)}$.

Obviously, $\mu_{1}, \mu_{2} \in \mathbb{R}$ and $\mu_{1} \neq \mu_{2}$, as expected. Moreover, the real symmetry of the hyperelliptic curve $\mathscr{K}_{2}$ implies that

$\mathscr{R}\left(\mu_{i}\right)=\left|\mu_{i}-\lambda_{1}\right|^{2}\left|\mu_{i}-\lambda_{3}\right|^{2}\left|\mu_{i}-\lambda_{5}\right|^{2}$,

since $\mu_{i} \in \mathbb{R}$ for $i=1,2$.

The explicit expressions of Theorem 5 can now be used to obtain the result, using standard results of differential calculus. Eq. (27) is also used, in order to determine the correct hyperelliptic sheets, $\sigma_{1}= \pm 1$ or $\sigma_{2}= \pm 1$, for the points $\left(\mu_{1}, \sigma_{1} \sqrt{\mathscr{R}\left(\mu_{1}\right)}\right)$ and $\left(\mu_{2}, \sigma_{2} \sqrt{\mathscr{R}\left(\mu_{2}\right)}\right)$ on the Riemann surface $\mathscr{K}_{2}$. Eq. (60) becomes

$$
\begin{aligned}
\frac{\partial^{2} v_{1}}{\partial x^{2}} \frac{\partial^{2} v_{1}}{\partial t^{2}}-\left(\frac{\partial^{2} v_{1}}{\partial x \partial t}\right)^{2}= & 256 v_{1}^{2} \Psi_{11}\left(\mu_{1}\right) \Psi_{11}\left(\mu_{2}\right) \\
= & -256 v_{1}^{2} \sigma_{1} \sigma_{2} \sqrt{\mathscr{R}\left(\mu_{1}\right)} \sqrt{\mathscr{R}\left(\mu_{2}\right)} \\
= & 256\left(s_{1}+s_{2}+s_{3}\right) s_{1} s_{2} s_{3}\left|\lambda_{1}-\lambda_{3}^{*}\right|^{2} \\
& \times\left|\lambda_{1}-\lambda_{5}^{*}\right|^{2}\left|\lambda_{3}-\lambda_{5}^{*}\right|^{2} \\
> & 0 .
\end{aligned}
$$

Thus $\sigma_{1} \sigma_{2}<0$ and $\sigma_{2}=-\sigma_{1}$.

Eq. (61) becomes

$$
\begin{aligned}
\frac{\partial^{2} v_{1}}{\partial x^{2}}= & 8 i v_{1} \frac{\Psi_{11}\left(\mu_{1}\right)-\Psi_{11}\left(\mu_{2}\right)}{\mu_{1}-\mu_{2}} \\
= & 8 v_{1} \frac{s_{1}+s_{2}+s_{3}}{\sqrt{A(\vec{r}, \vec{s})}} \sigma_{1}\left(\sqrt{\mathscr{R}\left(\mu_{1}\right)}+\sqrt{\mathscr{R}\left(\mu_{2}\right)}\right) \\
= & -32\left(s_{1}+s_{2}+s_{3}\right) s_{1} s_{2} s_{3}-8 s_{1} s_{2}\left|\lambda_{1}-\lambda_{3}^{*}\right|^{2} \\
& -8 s_{1} s_{3}\left|\lambda_{1}-\lambda_{5}^{*}\right|^{2}-8 s_{2} s_{3}\left|\lambda_{3}-\lambda_{5}^{*}\right|^{2} \\
< & 0 .
\end{aligned}
$$

Hence there is a relative maximum. Moreover, at this relative maximum $\sigma_{1}=-1$ and $\sigma_{2}=1$.

Finally, notice that all the arguments in the proof and the final formulas are symmetric with respect to the labeling of the branch points.
Lemma 19. Suppose $\lambda_{1}=\lambda_{2}^{*}=r_{1}+i s_{1}, \lambda_{3}=\lambda_{4}^{*}=r_{2}+i s_{2}$ and $\lambda_{5}=\lambda_{6}^{*}=r_{3}+i s_{3}$, with $0<s_{1} \leq s_{2} \leq s_{3}$. If a smooth two-phase solution of the NLS equation (1) exists in a neighborhood of a point $\left(x, t, v_{1}(x, t)\right)=\left(x, t, v_{1}^{(i)}\right)$ for $i=2$ or $i=3$, then $\left(x, t, v_{1}^{(i)}\right)$ is a saddle point of $v_{1}=v_{1}(x, t)$.

Proof. Consider the case where $i=3$, the case for $i=2$ is similar. As in the previous lemma,

$$
\hat{\Sigma}_{2}=-\frac{\tilde{A}}{2\left(-s_{1}+s_{2}+s_{3}\right)^{2}},
$$

where $\tilde{A}=A\left(\vec{r},-s_{1}, s_{2}, s_{3}\right)$ and $A(\vec{r}, \vec{s})$ is given by Eq. (70). In this case the sign of $\tilde{A}=A\left(\vec{r},-s_{1}, s_{2}, s_{3}\right)$ is indeterminate, and

$$
\begin{aligned}
& \mu_{1}=\frac{r_{1}\left(s_{2}+s_{3}\right)+r_{2}\left(-s_{1}+s_{3}\right)+r_{3}\left(-s_{1}+s_{2}\right)-\sqrt{\tilde{A}}}{2\left(-s_{1}+s_{2}+s_{3}\right)}, \\
& \mu_{2}=\frac{r_{1}\left(s_{2}+s_{3}\right)+r_{2}\left(-s_{1}+s_{3}\right)+r_{3}\left(-s_{1}+s_{2}\right)+\sqrt{\tilde{A}}}{2\left(-s_{1}+s_{2}+s_{3}\right)} .
\end{aligned}
$$

If $\tilde{A} \neq 0$, then $\mu_{1} \neq \mu_{2}$ and the formula of Eq. (60) is applicable. If $\tilde{A}=0$, then $\mu_{1}=\mu_{2} \in \mathbb{R}$, but all the expressions for the secondorder partial derivatives of $\nu_{1}(x, t)$ have finite limits as $\mu_{2} \rightarrow \mu_{1}$ with $\mu_{2}^{*}=\mu_{1}$. So the formula of Eq. (60) is still valid in the limit. In particular, regardless of the value of $\tilde{A}$,

$$
\begin{aligned}
\frac{\partial^{2} v_{1}}{\partial x^{2}} \frac{\partial^{2} v_{1}}{\partial t^{2}}-\left(\frac{\partial^{2} v_{1}}{\partial x \partial t}\right)^{2}= & 256 v_{1}^{2} \Psi_{11}\left(\mu_{1}\right) \Psi_{11}\left(\mu_{2}\right) \\
= & -256\left(-s_{1}+s_{2}+s_{3}\right) s_{1} s_{2} s_{3} \\
& \times\left|\lambda_{1}-\lambda_{3}\right|^{2}\left|\lambda_{1}-\lambda_{5}\right|^{2}\left|\lambda_{3}-\lambda_{5}^{*}\right|^{2} \\
< & 0,
\end{aligned}
$$

so there is a saddle point. Notice that the assumed order of the imaginary parts of the branch points was used in the proof.

Lemma 20. Suppose $\lambda_{1}=\lambda_{2}^{*}=r_{1}+i s_{1}, \lambda_{3}=\lambda_{4}^{*}=r_{2}+i s_{2}$ and $\lambda_{5}=\lambda_{6}^{*}=r_{3}+i s_{3}$, with $0<s_{1} \leq s_{2} \leq s_{3}$. If a smooth two-phase solution of the NLS equation (1) exists in a neighborhood of a point $\left(x, t, v_{1}(x, t)\right)=\left(x, t, v_{1}^{(1)}\right)$, then $v_{1}=v_{1}(x, t)$ has a saddle point at $\left(x, t, v_{1}^{(1)}\right)$ when $s_{3}<s_{1}+s_{2}$, but $v_{1}(x, t)$ has a relative minimum of $v_{1}=v_{1}^{(1)}>0$ when $s_{3}>s_{1}+s_{2}$.

Proof. In this case

$$
\hat{\Sigma}_{2}=-\frac{\tilde{A}}{2\left(s_{1}+s_{2}-s_{3}\right)^{2}},
$$

where $\tilde{A}=A\left(\vec{r}, s_{1}, s_{2},-s_{3}\right)$ and $A(\vec{r}, \vec{s})$ is given by Eq. (70). In particular,

$$
\begin{aligned}
\tilde{A}= & -4 s_{1} s_{2} s_{3}\left(s_{1}+s_{2}-s_{3}\right)+s_{1}^{2}\left(r_{2}-r_{3}\right)^{2}+s_{2}^{2}\left(r_{1}-r_{3}\right)^{2} \\
& +s_{3}^{2}\left(r_{1}-r_{2}\right)^{2}-2 s_{1} s_{3}\left(r_{2}-r_{3}\right)\left(r_{2}-r_{1}\right) \\
& -2 s_{2} s_{3}\left(r_{1}-r_{3}\right)\left(r_{1}-r_{2}\right)+2 s_{1} s_{2}\left(r_{3}-r_{1}\right)\left(r_{3}-r_{2}\right),
\end{aligned}
$$

which, in general, has indeterminate sign. However, in the case $s_{3}>s_{1}+s_{2}$, it can be shown that $\tilde{A}>0$. Obviously the first term $\tilde{A}$ is positive in this case, however, the expression for $\tilde{A}$ is no longer symmetric with respect to the indices, so there are three cases to consider.

(i) If $r_{2} \leq r_{1} \leq r_{3}$ or $r_{3} \leq r_{1} \leq r_{2}$, then

$$
\begin{aligned}
\tilde{A}= & -4 s_{1} s_{2} s_{3}\left(s_{1}+s_{2}-s_{3}\right) \\
& +\left(s_{1}\left(r_{2}-r_{3}\right)+s_{3}\left(r_{1}-r_{2}\right)\right)^{2} \\
& +s_{2}^{2}\left(r_{1}-r_{3}\right)^{2}-2 s_{2} s_{3}\left(r_{1}-r_{3}\right)\left(r_{1}-r_{2}\right)
\end{aligned}
$$




$$
+2 s_{1} s_{2}\left(r_{3}-r_{1}\right)\left(r_{3}-r_{2}\right)
$$$$
>0 \text {, }
$$

since the first term is positive and the remaining terms are non-negative.

(ii) If $r_{1} \leq r_{2} \leq r_{3}$ or $r_{3} \leq r_{2} \leq r_{1}$, then

$$
\begin{aligned}
\tilde{A}= & -4 s_{1} s_{2} s_{3}\left(s_{1}+s_{2}-s_{3}\right) \\
& +\left(s_{2}\left(r_{1}-r_{3}\right)-s_{3}\left(r_{1}-r_{2}\right)\right)^{2} \\
& +s_{1}^{2}\left(r_{2}-r_{3}\right)^{2}-2 s_{1} s_{3}\left(r_{2}-r_{3}\right)\left(r_{2}-r_{1}\right) \\
& +2 s_{1} s_{2}\left(r_{3}-r_{1}\right)\left(r_{3}-r_{2}\right)
\end{aligned}
$$$$
>0 \text {, }
$$

since the first term is positive and the remaining terms are non-negative.

(iii) If $r_{1} \leq r_{3} \leq r_{2}$ or $r_{2} \leq r_{3} \leq r_{1}$, then

$$
\begin{aligned}
\tilde{A}= & -4 s_{1} s_{2} s_{3}\left(s_{1}+s_{2}-s_{3}\right)+s_{1}^{2}\left(r_{2}-r_{3}\right)^{2} \\
& +s_{2}^{2}\left(r_{1}-r_{3}\right)^{2}+s_{3}^{2}\left(r_{1}-r_{2}\right)^{2}-2 s_{1} s_{3}\left(r_{2}-r_{3}\right) \\
& \times\left(r_{2}-r_{1}\right)-2 s_{2} s_{3}\left(r_{1}-r_{3}\right)\left(r_{1}-r_{2}\right) \\
& +2 s_{1} s_{2}\left(r_{3}-r_{1}\right)\left(r_{3}-r_{2}\right) \\
> & a s_{1}^{2}+b s_{1}+c,
\end{aligned}
$$

where, by the assumption of Theorem $6,0<s_{1} \leq s_{2}$, and

$$
\begin{aligned}
a= & \left(r_{2}-r_{3}\right)^{2} \geq 0, \\
b= & -2 s_{3}\left(r_{2}-r_{3}\right)\left(r_{2}-r_{1}\right) \\
& +2 s_{2}\left(r_{3}-r_{1}\right)\left(r_{3}-r_{2}\right) \leq 0, \\
c= & \left(s_{2}\left(r_{1}-r_{3}\right)-s_{3}\left(r_{1}-r_{2}\right)\right)^{2} \geq 0 .
\end{aligned}
$$

Consider the quadratic polynomial $f\left(s_{1}\right)=a s_{1}^{2}+b s_{1}+c$ on the interval $0 \leq s_{1} \leq s_{2}$. Clearly $f(0)=c \geq 0$. When $s_{1}=s_{2}$, it is also true that $s_{3}>s_{1}+s_{2}=2 s_{2}$, so

$f\left(s_{2}\right)=s_{2}^{2}\left(r_{1}+r_{2}-2 r_{3}\right)^{2}+s_{3}\left(s_{3}-2 s_{2}\right)\left(r_{1}-r_{2}\right)^{2} \geq 0$.

If $r_{2}=r_{3}$, then the graph of $y=f\left(s_{1}\right)$ is a straight line and $f\left(s_{1}\right) \geq 0$ for all $0 \leq s_{1} \leq s_{2}$. If $r_{2} \neq r_{3}$, then the minimum of $f\left(s_{1}\right)$ occurs at

$s_{1 m}=\frac{s_{2}\left(r_{3}-r_{1}\right)+s_{3}\left(r_{2}-r_{1}\right)}{r_{2}-r_{3}}>0$.

(1) In the case $r_{1} \leq r_{3}<r_{2}$, we can use $s_{3}>s_{1}+s_{2}$ to show that

$$
\begin{aligned}
s_{1 m} & =\frac{s_{2}\left(r_{3}-r_{1}\right)+s_{3}\left(r_{2}-r_{1}\right)}{r_{2}-r_{3}} \\
& >\frac{s_{2}\left(r_{3}-r_{1}\right)+\left(s_{1}+s_{2}\right)\left(r_{2}-r_{1}\right)}{r_{2}-r_{3}} \\
& =\frac{s_{1}\left(r_{2}-r_{1}\right)+2 s_{2}\left(r_{3}-r_{1}\right)+s_{2}\left(r_{2}-r_{3}\right)}{r_{2}-r_{3}} \\
& =s_{2}+\frac{s_{1}\left(r_{2}-r_{1}\right)+2 s_{2}\left(r_{3}-r_{1}\right)}{r_{2}-r_{3}} \\
& \geq s_{2} .
\end{aligned}
$$

(2) In the case $r_{2}<r_{3} \leq r_{1}$, we can similarly show, using $s_{3}>s_{1}+s_{2}$, that

$$
\begin{aligned}
s_{1 m} & =\frac{s_{2}\left(r_{1}-r_{3}\right)+s_{3}\left(r_{1}-r_{2}\right)}{r_{3}-r_{2}} \\
& >\frac{s_{2}\left(r_{1}-r_{3}\right)+\left(s_{1}+s_{2}\right)\left(r_{1}-r_{2}\right)}{r_{3}-r_{2}} \\
& =\frac{s_{1}\left(r_{1}-r_{2}\right)+2 s_{2}\left(r_{1}-r_{3}\right)+s_{2}\left(r_{3}-r_{2}\right)}{r_{3}-r_{2}} \\
& =s_{2}+\frac{s_{1}\left(r_{1}-r_{2}\right)+2 s_{2}\left(r_{1}-r_{3}\right)}{r_{3}-r_{2}} \\
& \geq s_{2} .
\end{aligned}
$$

In both cases, $s_{1 m}>s_{2}$, so, on the interval $0 \leq s_{1} \leq s_{2}, f\left(s_{1}\right) \geq$ 0 . Therefore

$$
\tilde{A}>a s_{1}^{2}+b s_{1}+c \geq 0 \text {. }
$$

Thus, in all cases when $s_{3}>s_{1}+s_{2}, \tilde{A}>0$ and, hence, $\hat{\Sigma}_{2}<0$.

The expressions for $\mu_{1}$ and $\mu_{2}$ are similar to those obtained in the previous lemma,

$\mu_{1}=\frac{r_{1}\left(s_{2}-s_{3}\right)+r_{2}\left(s_{1}-s_{3}\right)+r_{3}\left(s_{1}+s_{2}\right)-\sqrt{\tilde{A}}}{2\left(s_{1}+s_{2}-s_{3}\right)}$,
$\mu_{2}=\frac{r_{1}\left(s_{2}-s_{3}\right)+r_{2}\left(s_{1}-s_{3}\right)+r_{3}\left(s_{1}+s_{2}\right)+\sqrt{\tilde{A}}}{2\left(s_{1}+s_{2}-s_{3}\right)}$,

so that when $s_{3}>s_{1}+s_{2}, \tilde{A}>0$ implies $\mu_{1}, \mu_{2} \in \mathbb{R}$ and $\mu_{1} \neq \mu_{2}$.

The formula given by Eq. (60) implies that when $v_{1}=v_{1}^{(1)}$,

$$
\begin{aligned}
\frac{\partial^{2} v_{1}}{\partial x^{2}} \frac{\partial^{2} v_{1}}{\partial t^{2}}-\left(\frac{\partial^{2} v_{1}}{\partial x \partial t}\right)^{2}= & 256 v_{1}^{2} \Psi_{11}\left(\mu_{1}\right) \Psi_{11}\left(\mu_{2}\right) \\
= & -256\left(s_{1}+s_{2}-s_{3}\right) s_{1} s_{2} s_{3} \\
& \times\left|\lambda_{1}-\lambda_{3}^{*}\right|^{2}\left|\lambda_{1}-\lambda_{5}\right|^{2}\left|\lambda_{3}-\lambda_{5}\right|^{2} .
\end{aligned}
$$

If $s_{3}<s_{1}+s_{2}$, then the above expression is negative, so there is a saddle point. If $s_{3}>s_{1}+s_{2}$, then the above expression is positive. Also $\tilde{A}>0$ and $\mu_{1} \neq \mu_{2}$, so Eq. (61) becomes

$$
\begin{aligned}
\frac{\partial^{2} v_{1}}{\partial x^{2}}= & 8 i v_{1} \frac{\Psi_{11}\left(\mu_{1}\right)-\Psi_{11}\left(\mu_{2}\right)}{\mu_{1}-\mu_{2}} \\
= & 32\left(s_{1}+s_{2}-s_{3}\right) s_{1} s_{2} s_{3}-8 s_{1} s_{2}\left|\lambda_{1}-\lambda_{3}^{*}\right|^{2} \\
& +8 s_{1} s_{3}\left|\lambda_{1}-\lambda_{5}\right|^{2}+8 s_{2} s_{3}\left|\lambda_{3}-\lambda_{5}\right|^{2} \\
= & 8\left(s_{1}+s_{2}\right)\left(s_{3}-s_{1}\right)\left(s_{3}-s_{2}\right)\left(s_{3}-s_{1}-s_{2}\right) \\
& -8 s_{1} s_{2}\left(r_{1}-r_{2}\right)^{2}+8 s_{2} s_{3}\left(r_{2}-r_{3}\right)^{2} \\
& +8 s_{1} s_{3}\left(r_{1}-r_{3}\right)^{2} \\
> & 8\left(s_{1}+s_{2}\right)\left(s_{3}-s_{1}\right)\left(s_{3}-s_{2}\right)\left(s_{3}-s_{1}-s_{2}\right) \\
& -8 s_{1} s_{2}\left(r_{1}-r_{2}\right)^{2}+8 s_{2}\left(s_{1}+s_{2}\right)\left(r_{2}-r_{3}\right)^{2} \\
& +8 s_{1}\left(s_{1}+s_{2}\right)\left(r_{1}-r_{3}\right)^{2} \\
= & 8\left(s_{1}+s_{2}\right)\left(s_{3}-s_{1}\right)\left(s_{3}-s_{2}\right)\left(s_{3}-s_{1}-s_{2}\right) \\
& +8\left(s_{2}\left(r_{2}-r_{3}\right)+s_{1}\left(r_{1}-r_{3}\right)\right)^{2} \\
> & 0
\end{aligned}
$$

so $v_{1}=v_{1}^{(1)}$ is a relative minimum. Clearly, the assumed order of the imaginary parts was crucial to the result.

Lemma 21. Suppose $\lambda_{1}=\lambda_{2}^{*}=r_{1}+i s_{1}, \lambda_{3}=\lambda_{4}^{*}=r_{2}+i s_{2}$ and $\lambda_{5}=\lambda_{6}^{*}=r_{3}+i s_{3}$, with $0<s_{1} \leq s_{2} \leq s_{3}$. If $v_{1}^{(i)}>0$, then the four real points $\left(v_{1}^{(i)}, v_{2}^{(i)}\right), i=1, \ldots, 4$, given in Theorem 6 , satisfying $\hat{\Sigma}_{3}=0$ and $\hat{\Sigma}_{2}^{2}-4 \hat{\Sigma}_{4}=0$, are singular points of a one-dimensional algebraic set defined by Eq. (49),

$16 \hat{\Sigma}_{4}\left(\hat{\Sigma}_{2}^{2}-4 \hat{\Sigma}_{4}\right)^{2}-\hat{\Sigma}_{3}^{2}\left(4 \hat{\Sigma}_{2}^{3}-144 \hat{\Sigma}_{2} \hat{\Sigma}_{4}+27 \hat{\Sigma}_{3}^{2}\right)=0$.

At $\left(v_{1}, v_{2}\right)=\left(v_{1}^{(i)}, v_{2}^{(i)}\right)$, for $i=1,2$, and 3, if $\hat{\Sigma}_{2}=-2 \sqrt{\hat{\Sigma}_{4}}<0$, then $\left(v_{1}^{(i)}, v_{2}^{(i)}\right)$ is a node, but if $\hat{\Sigma}_{2}=2 \sqrt{\hat{\Sigma}_{4}}>0$, then $\left(v_{1}^{(i)}, v_{2}^{(i)}\right)$ is an isolated point. In particular, when $s_{3}>s_{1}+s_{2}$, at $\left(v_{1}, v_{2}\right)=$ $\left(v_{1}^{(1)}, v_{2}^{(1)}\right), \hat{\Sigma}_{2}=-2 \sqrt{\hat{\Sigma}_{4}}<0$, and $\left(v_{1}^{(1)}, v_{2}^{(1)}\right)$ is a node. However, at $\left(v_{1}, v_{2}\right)=\left(v_{1}^{(4)}, v_{2}^{(4)}\right)$ it is always true that $\hat{\Sigma}_{2}=-2 \sqrt{\hat{\Sigma}_{4}}<0$, hence $\left(v_{1}^{(4)}, v_{2}^{(4)}\right)$ is always a node.

Proof. When $v_{1}>0$, Eq. (92) defines an algebraic set given by a polynomial equation $Q\left(v_{1}, v_{2}\right)=0$, in which $Q$ is of degree 9 
in $v_{1}$ and degree 8 in $v_{2}$. At $\left(v_{1}, v_{2}\right)=\left(v_{1}^{(4)}, v_{2}^{(4)}\right)$, substitution and simplification, using a computer algebra system such as Maple, shows that $Q=0, Q_{v_{1}}=0, Q_{v_{2}}=0$, and

$Q_{v_{1} v_{1}} Q_{v_{2} \nu_{2}}-Q_{v_{1} \nu_{2}}^{2}=K s_{1}^{2} s_{2}^{2} s_{3}^{2}\left(s_{1}+s_{2}+s_{3}\right)^{10} a_{1}^{2} a_{2}^{2} a_{3}^{2} \hat{\Sigma}_{2}^{5}$,

where $K>0$ is a positive constant and

$a_{1}=\left(r_{2}-r_{3}\right)^{2}+\left(s_{2}+s_{3}\right)^{2}$,

$a_{2}=\left(r_{1}-r_{3}\right)^{2}+\left(s_{1}+s_{3}\right)^{2}$,

$a_{3}=\left(r_{2}-r_{1}\right)^{2}+\left(s_{1}+s_{2}\right)^{2}$.

Clearly $a_{1}, a_{2}$, and $a_{3}$ are all strictly positive. In Lemma 18 , it was shown that $\hat{\Sigma}_{2}=-2 \hat{\Sigma}_{4}<0$ at $\left(v_{1}, v_{2}\right)=\left(v_{1}^{(4)}, v_{2}^{(4)}\right)$. Hence, at $\left(v_{1}, v_{2}\right)=\left(v_{1}^{(4)}, v_{2}^{(4)}\right)$

$Q_{v_{1} v_{1}} Q_{v_{2} \nu_{2}}-Q_{v_{1} \nu_{2}}^{2}<0$

and there is always a node at this singular point.

In the case of each of the other singular points $\left(v_{1}^{(i)}, v_{2}^{(i)}\right), i=$ $1,2,3$, Eqs. (93) and (94) remain the same, except that the sign in front of $s_{1}$ changes from positive to negative for $i=3$, the sign in front of $s_{2}$ changes from positive to negative for $i=2$, and the sign in front of $s_{3}$ changes from positive to negative for $i=1$. Thus the sign of the expression on the right-hand side of Eq. (93) is always the opposite of the sign of $\hat{\Sigma}_{2}$. Therefore, when $\hat{\Sigma}_{2}>0$, there is an isolated point, but when $\hat{\Sigma}_{2}<0$, there is a node.

In particular, the proof of Lemma 20 shows that, when $s_{3}>$ $s_{1}+s_{2}, \hat{\Sigma}_{2}<0$, so that when $s_{3}>s_{1}+s_{2}$, the point $\left(v_{1}^{(1)}, v_{2}^{(1)}\right)$ is a node.

Lemma 22. Suppose $\lambda_{1}=\lambda_{2}^{*}=r_{1}+i s_{1}, \lambda_{3}=\lambda_{4}^{*}=r_{2}+i s_{2}$ and $\lambda_{5}=\lambda_{6}^{*}=r_{3}+i s_{3}$, with $s_{1}, s_{2}, s_{3}>0$. The common root $z>0$ of Eqs. (44) and (51) that exists at each point of the algebraic set (92), provided $\hat{\Sigma}_{3} \neq 0$, has a positive limit $z \rightarrow-\hat{\Sigma}_{2}>0$ at the node $\left(v_{1}^{(4)}, v_{2}^{(4)}\right)$, where $\hat{\Sigma}_{3}=0$ and $\hat{\Sigma}_{2}=-2 \sqrt{\hat{\Sigma}_{4}}<0$. Moreover, the same point $\left(v_{1}^{(4)}, v_{2}^{(4)}\right)$ is a regular point of each of the two distinct algebraic sets $\rho_{+}=0$ and $\rho_{-}=0$ which cross transversely as subsets of the algebraic set (92) at the node at $\left(v_{1}^{(4)}, v_{2}^{(4)}\right)$.

Proof. Lemma 18 shows that $\hat{\Sigma}_{2}=-2 \sqrt{\hat{\Sigma}_{2}}<0$ at $\left(v_{1}^{(4)}, v_{2}^{(4)}\right)$. Since $\hat{\Sigma}_{3}=0$ at this point, Eqs. (44) and (51) have two common roots, $z=0$ (multiplicity two) and $z=-\hat{\Sigma}_{2}$ (multiplicity one). Therefore, the common root $z>0$ of Eqs. (44) and (51) that exists at each point of the algebraic set (92), provided $\hat{\Sigma}_{3} \neq 0$, must approach either 0 or $-\hat{\Sigma}_{2}$. The expression for this common root is given in Eq. (50), and since $\hat{\Sigma}_{2}<0, z \rightarrow-\hat{\Sigma}_{2}>0$. Thus the singularity in $\rho_{+}$and $\rho_{-}$at $\left(v_{1}^{(4)}, v_{2}^{(4)}\right)$ is removable.

Direct calculation shows that, at $\left(v_{1}^{(4)}, v_{2}^{(4)}\right)$,

$$
\begin{aligned}
\hat{\Sigma}_{3, \nu_{2}}= & \frac{1}{2\left(s_{1}+s_{2}+s_{3}\right)^{4}}\left(\left(s_{1}+s_{2}\right)\left(s_{1}+s_{3}\right)\left(s_{2}+s_{3}\right)\right. \\
& \times\left(s_{1}+s_{2}+s_{3}\right)+s_{1} s_{2}\left(r_{1}-r_{2}\right)^{2}+s_{1} s_{3}\left(r_{1}-r_{3}\right)^{2} \\
& \left.+s_{2} s_{3}\left(r_{2}-r_{3}\right)^{2}\right) \\
> & 0,
\end{aligned}
$$

and

$\rho_{ \pm, v_{2}}=\hat{\Sigma}_{2, v_{2}}+z_{v_{2}} \pm \frac{1}{\sqrt{z}} \hat{\Sigma}_{3, v_{2}}$

So at least one of $\rho_{ \pm, v_{2}}$ is nonzero. Thus $\left(v_{1}^{(4)}, v_{2}^{(4)}\right)$ is a regular point of at least one of $\rho_{+}=0$ or $\rho_{-}=0$. Since the same point is also a node of the algebraic set (92), in a neighborhood of which $z>0$ and $\rho_{+} \rho_{-}=0$ on the algebraic set, the two transverse onedimensional submanifolds of the algebraic set at the node must correspond to $\rho_{+}=0$ and $\rho_{-}=0$.

Lemma 23. Suppose $\lambda_{1}=\lambda_{2}^{*}=r_{1}+i s_{1}, \lambda_{3}=\lambda_{4}^{*}=r_{2}+i s_{2}$ and $\lambda_{5}=\lambda_{6}^{*}=r_{3}+i s_{3}$, with $0<s_{1} \leq s_{2} \leq s_{3}$. When $s_{3}>s_{1}+s_{2}$, the common root $z>0$ of Eqs. (44) and (51) that exists at each point of the algebraic set (92), provided $\hat{\Sigma}_{3} \neq 0$, has a positive limit $z=-\hat{\Sigma}_{2}>0$ at the node $\left(v_{1}^{(1)}, v_{2}^{(1)}\right)$, where $\hat{\Sigma}_{3}=0$ and $\hat{\Sigma}_{2}=-2 \sqrt{\hat{\Sigma}_{4}}<0$. Moreover, the same point $\left(v_{1}^{(1)}, v_{2}^{(1)}\right)$ is a regular point of each of the two distinct algebraic sets $\rho_{+}=0$ and $\rho_{-}=0$ which cross transversely as subsets of the algebraic set (92) at the node $\left(v_{1}^{(1)}, v_{2}^{(1)}\right)$.

Proof. Lemma 20 shows that, when $s_{3}>s_{1}+s_{2}, \hat{\Sigma}_{2}=-2 \sqrt{\hat{\Sigma}_{4}}<$ 0 at $\left(v_{1}^{(1)}, v_{2}^{(1)}\right)$. As in the previous lemma, the expression for the common root $z>0$ on the boundary is given by Eq. (50), and since $\hat{\Sigma}_{2}<0, z \rightarrow-\hat{\Sigma}_{2}>0$. Thus the singularity in $\rho_{+}$and $\rho_{-}$at $\left(v_{1}^{(1)}, v_{2}^{(1)}\right)$ is removable.

Direct calculation shows that, at $\left(v_{1}^{(1)}, v_{2}^{(1)}\right)$,

$$
\begin{aligned}
\hat{\Sigma}_{3, v_{2}}= & \frac{1}{2\left(s_{1}+s_{2}-s_{3}\right)^{4}}\left(\left(s_{1}+s_{2}\right)\left(s_{1}-s_{3}\right)\left(s_{2}-s_{3}\right)\right. \\
& \times\left(s_{1}+s_{2}-s_{3}\right)+s_{1} s_{2}\left(r_{1}-r_{2}\right)^{2}-s_{1} s_{3}\left(r_{1}-r_{3}\right)^{2} \\
& \left.-s_{2} s_{3}\left(r_{2}-r_{3}\right)^{2}\right) \\
< & \frac{1}{2\left(s_{1}+s_{2}-s_{3}\right)^{4}}\left(\left(s_{1}+s_{2}\right)\left(s_{1}-s_{3}\right)\left(s_{2}-s_{3}\right)\right. \\
& \times\left(s_{1}+s_{2}-s_{3}\right)+s_{1} s_{2}\left(r_{1}-r_{2}\right)^{2} \\
& \left.-s_{1}\left(s_{1}+s_{2}\right)\left(r_{1}-r_{3}\right)^{2}-s_{2}\left(s_{1}+s_{2}\right)\left(r_{2}-r_{3}\right)^{2}\right) \\
= & \frac{1}{2\left(s_{1}+s_{2}-s_{3}\right)^{4}}\left(\left(s_{1}+s_{2}\right)\left(s_{1}-s_{3}\right)\left(s_{2}-s_{3}\right)\right. \\
& \times\left(s_{1}+s_{2}-s_{3}\right)-s_{1}^{2}\left(r_{1}-r_{3}\right)^{2} \\
& \left.-2 s_{1} s_{2}\left(r_{2}-r_{3}\right)\left(r_{1}-r_{3}\right)-s_{2}^{2}\left(r_{2}-r_{3}\right)^{2}\right) \\
= & \frac{1}{2\left(s_{1}+s_{2}-s_{3}\right)^{4}}\left(\left(s_{1}+s_{2}\right)\left(s_{1}-s_{3}\right)\left(s_{2}-s_{3}\right)\right. \\
& \left.\times\left(s_{1}+s_{2}-s_{3}\right)-\left(s_{1}\left(r_{1}-r_{3}\right)+s_{2}\left(r_{2}-r_{3}\right)\right)^{2}\right) \\
< & 0
\end{aligned}
$$

since $s_{3}>s_{1}+s_{2}$. Also

$\rho_{ \pm, \nu_{2}}=\hat{\Sigma}_{2, \nu_{2}}+z_{\nu_{2}} \pm \frac{1}{\sqrt{z}} \hat{\Sigma}_{3, v_{2}}$

So at least one of $\rho_{ \pm, v_{2}}$ is nonzero at $\left(v_{1}^{(1)}, v_{2}^{(1)}\right)$ when $s_{3}>s_{1}+s_{2}$. Thus $\left(v_{1}^{(1)}, v_{2}^{(1)}\right)$ is a regular point of at least one of $\rho_{+}=0$ or $\rho_{-}=0$. Since the same point is also a node of the algebraic set (92), in a neighborhood of which $z>0$ and $\rho_{+} \rho_{-}=0$ on the algebraic set, the two transverse one-dimensional submanifolds of the algebraic set at the node must correspond to $\rho_{+}=0$ and $\rho_{-}=0$.

Lemma 24. Suppose $\lambda_{1}=\lambda_{2}^{*}=r_{1}+i s_{1}, \lambda_{3}=\lambda_{4}^{*}=r_{2}+i s_{2}$ and $\lambda_{5}=\lambda_{6}^{*}=r_{3}+i s_{3}$, with $0<s_{1} \leq s_{2} \leq s_{3}$. If $s_{3}>s_{1}+s_{2}$, then for each point $\left(0, v_{2}\right)$ in the $v_{1}-v_{2}$-plane there is some open neighborhood of that point such that, for each point in that neighborhood for which $v_{1}>0$, the reality constraint of Theorem 1, viz., the Dirichlet eigenvalues come in complexconjugate pairs, cannot be true. Thus, when $s_{3}>s_{1}+s_{2}$, there is no smooth two-phase solution whose minimum value of $v_{1}$ is zero. 
Proof. Theorem 3 says that when $v_{1}>0$, the boundary of the set of points $\left(v_{1}, v_{2}\right)$ for which the reality condition is satisfied lies on an algebraic set defined by Eq. (49), viz., a polynomial equation $Q\left(v_{1}, v_{2}\right)=0$, in which $Q$ is of degree 9 in $v_{1}$ and degree 8 in $v_{2}$, obtained by clearing the denominators of factors of $v_{1}$. For connected regions separated by the boundary, whether or not the reality condition is satisfied is well-defined.

The nature of this algebraic set near $(0,0)$ will be important to understand. In particular, $Q(0,0)=0$, and

$$
\begin{aligned}
Q_{\nu_{1}}(0,0)= & 2^{15} a_{-1}^{3}\left(\left(s_{1}+s_{2}\right)^{2}-s_{3}^{2}\right)\left(s_{1}-s_{2}+s_{3}\right) \\
& \times\left(s_{1}-s_{2}-s_{3}\right) \cdot\left(s_{1}^{2}+\left(r_{2}-r_{3}\right)^{2}\right) \\
& \times\left(s_{2}^{2}+\left(r_{1}-r_{3}\right)^{2}\right)\left(s_{3}^{2}+\left(r_{1}-r_{2}\right)^{2}\right),
\end{aligned}
$$

where $a_{-1}$ is a degree-four homogeneous polynomial in the variables $r_{1}, r_{2}, r_{3}, s_{1}, s_{2}, s_{3}$, which also occurs in the asymptotic expansion of $z$ given below. Notice that if $s_{1}+s_{2}<s_{3}$ and $a_{-1} \neq 0$, then $Q_{v_{1}} \neq 0$, and the implicit function theorem implies that $v_{1}$ is a locally well-defined function of $v_{2}$ near $\left(v_{1}, v_{2}\right)=(0,0)$, which defines the curve, tangential to the line $\nu_{1}=0$, along which $\rho_{+}$ and $\rho_{-}$can change sign in the neighborhood of $(0,0)$.

However, if $a_{-1}=0$, then $Q_{v_{1}}(0,0)=0$. Also $Q_{v_{2}}(0,0)=$ $Q_{v_{2} \nu_{2}}(0,0)=0$, but $A=Q_{v_{1} v_{1}}(0,0) \neq 0$ and $B=Q_{v_{2} v_{2} v_{2}}(0,0) \neq$ 0 , because the resultant of $a_{-1}$ and $A$ and the resultant of $a_{-1}$ and $B$, as polynomials in $r_{1}$, are both nonzero. Therefore, when $a_{-1}=0$, there is a cusp at $(0,0)$ of the form

$Q=A v_{1}^{2}+B v_{2}^{3}+O\left(v_{1}^{3}, v_{1}^{2} v_{2}, v_{1} v_{2}^{2}\right)$.

In this case, there is a region in which $v_{2} \neq 0$ and $v_{1}>0$ which is not separated from the line $v_{2}=0$ by the cusp.

Now consider the various cases that may occur as $v_{1} \rightarrow 0$.

1. If $v_{2} \neq 0$, then, as $v_{1} \rightarrow 0, \hat{\Sigma}_{3} \neq 0$, and there is a positive root $z$ of Eq. (44) with the following expansion in powers of $v_{1}$,

$z=\frac{v_{2}^{2}}{32 v_{1}^{2}}+O\left(\frac{1}{v_{1}}\right)$.

Using this value of $z$, we find that

$\rho_{+}=-\frac{v_{2}^{2}}{8 v_{1}^{2}}+O\left(\frac{1}{v_{1}}\right)$,

and $\rho_{-}=O(1)$. So, whenever $v_{2} \neq 0, \rho_{+}<0$ for $v_{1}$ sufficiently small. Since the asymptotic expansion can be made uniform in $v_{2}$ on any interval of $v_{2}$ bounded away $v_{2}=0$, it follows that when $v_{2} \neq 0$, there is no region of points satisfying the reality conditions that extends to $\left(0, v_{2}\right)$.

2. If $v_{2}=0$, then

$$
\hat{\Sigma}_{3}=\frac{b_{-1}}{v_{1}}+b_{0}+b_{1} v_{1}
$$

where $b_{-1}, b_{0}$, and $b_{1}$ are homogeneous polynomials of indeterminate sign and of degree five, three, and one, respectively, in the variables $r_{1}, r_{2}, r_{3}, s_{1}, s_{2}, s_{3}$.

(a) If $b_{-1} \neq 0$, then

$$
z=\frac{a_{-1}}{v_{1}}+O(1)
$$

where $a_{-1}$ is the same polynomial that appears in Eq. (100).

i. If $a_{-1}>0$, then Eq. (105) gives $z>0$, and

$$
\rho_{+}=-\frac{a_{-1}}{v_{1}}+O(1) \text {. }
$$

Similarly, $\rho_{-}$has the same leading-order behavior, so that both $\rho_{+}$and $\rho_{-}$are negative for $\nu_{2}=0$ and $\nu_{1}>0$ sufficiently small. Since $Q\left(v_{1}, v_{2}\right)=0$ is a well-defined smooth curve tangential to $v_{1}=0$ in the vicinity of
$(0,0)$, it follows that the reality condition cannot be satisfied on either side of this curve for $v_{1}>0$, and so no region of points satisfying the reality condition can extend to $(0,0)$.

ii. If $a_{-1}<0$, then Eq. (105) does not give a positive value of $z$, instead the positive root $z=O(1)$. In particular,

$z=\frac{b_{-1}^{2}}{8 a_{-1}^{2}}+O\left(v_{1}\right)$.

In this case,

$$
\begin{aligned}
& \rho_{+}=-\frac{c_{0}}{8 a_{-1}^{2}}+O\left(v_{1}\right), \\
& \rho_{-}=-\frac{4 a_{-1}}{v_{1}}+O(1),
\end{aligned}
$$

where

$$
\begin{aligned}
c_{0}= & \left(s_{3}^{2}-\left(s_{1}+s_{2}\right)^{2}\right)\left(s_{3}^{2}-\left(s_{1}-s_{2}\right)^{2}\right) \\
& \times\left(s_{1}^{2}+\left(r_{2}-r_{3}\right)^{2}\right) \cdot\left(s_{2}^{2}+\left(r_{1}-r_{3}\right)^{2}\right) \\
& \times\left(s_{3}^{2}+\left(r_{1}-r_{2}\right)^{2}\right) .
\end{aligned}
$$

Clearly $c_{0}>0$ when $s_{3}>s_{1}+s_{2}$, so that $\rho_{-}>0$ and $\rho_{+}<0$ as $v_{1} \rightarrow 0$. As in the case where $a_{-1}>0$, no region of points satisfying the reality condition can extend to $(0,0)$.

iii. If $a_{-1}=0$, then the curve defined by $Q=0$ has a cusp at $(0,0)$, and the line $v_{2}=0$ is on one side of this cusp which is connected to a region where $v_{2} \neq 0$ and $v_{1} \rightarrow 0$. Once again, the reality condition cannot be satisfied on either side of this cusp where $v_{1}>0$ in a neighborhood of $(0,0)$.

(b) If $b_{-1}=0$, then $a_{-1} \neq 0$, because the resultant of $a_{-1}$ and $b_{-1}$, as polynomials in $r_{1}$, is not zero. Therefore $Q=0$ defines a smooth curve tangential to the line $v_{1}=0$ near $(0,0)$. If $b_{0} \neq 0$, or if $b_{0}=0$ and $b_{1} \neq 0$, it can be shown by explicit calculation that the asymptotic series of both $\rho_{+}$ and $\rho_{-}$as $\nu_{1} \rightarrow 0$ are the same as calculated previously in the case where $b_{-1} \neq 0$. If $b_{0}=b_{1}=0$, then $\hat{\Sigma}_{3}=0$ identically on the line $v_{2}=0$, and $z$ is not defined. However, explicit calculation, using the expressions in Corollary 3 , shows that the reality constraint of Corollary 3 is not satisfied as $v_{1} \rightarrow 0$. In fact the asymptotic expansions of the corresponding expressions in Corollary 3 are the same as those for $\rho_{+}$and $\rho_{-}$when $b_{-1} \neq 0$. This result can be inferred from the continuity of the Dirichlet eigenvalues. In any case, the reality constraint is not satisfied on both sides of the curve $Q=0$ in some neighborhood of $(0,0)$, and no region of points satisfying the reality condition can extend to $(0,0)$.

Theorem 7. Suppose $\lambda_{1}=\lambda_{2}^{*}=r_{1}+i s_{1}, \lambda_{3}=\lambda_{4}^{*}=r_{2}+i s_{2}$ and $\lambda_{5}=\lambda_{6}^{*}=r_{3}+i s_{3}$, with $0<s_{1} \leq s_{2} \leq s_{3}$. The set of initial conditions for the Dirichlet eigenvalues $\mu_{1}$ and $\mu_{2}$ which satisfy the reality condition of Theorem 1 for the smooth two-phase solution $p=p(x, t)$ of the NLS equation (1) is parametrized by a single compact connected region of $\left\{\left(v_{1}, v_{2}\right) \in \mathbb{R}^{2}\right\}$. The maximum value of $v_{1}=|p(x, t)|^{2}$ on this region is $\left(s_{1}+s_{2}+s_{3}\right)^{2}$, and the minimum value of $v_{1}=|p(x, t)|^{2}$ on this region is $\left(s_{1}+s_{2}-s_{3}\right)^{2}>0$, if $s_{3}>s_{1}+s_{2}$, or zero, if $s_{3} \leq s_{1}+s_{2}$.

Proof. Lemma 17 shows that smooth two-phase solutions are bounded, and Theorem 6 enumerates the four possible nonzero bounds of $v_{1}$. Lemma 22 shows that $\rho_{+}=0$ and $\rho_{-}=0$ cross transversely at the point $\left(v_{1}, v_{2}\right)=\left(v_{1}^{(4)}, v_{2}^{(4)}\right)$, where $z>0$. These two one-dimensional algebraic sets divide a neighborhood 
of $\left(v_{1}, v_{2}\right)=\left(v_{1}^{(4)}, v_{2}^{(4)}\right)$ into four regions, exactly one of which (to the left of $\left(v_{1}, v_{2}\right)=\left(v_{1}^{(4)}, v_{2}^{(4)}\right)$, since $v_{1}^{(4)}$ must be a relative maximum, by Lemma 18) contains an open set where $\rho_{+}>$ $0, \rho_{-}>0$, and $z>0$. Thus there exists a region of initial conditions for $v_{1}$ and $v_{2}$ and, hence, for the Dirichlet eigenvalues $\mu_{1}$ and $\mu_{2}$, on which the reality condition is satisfied. Similarly, Lemma 23 shows that when $s_{3}>s_{1}+s_{2}$, there exists a region of permissible initial conditions for $v_{1}$ and $v_{2}$ in a sector to the right of the node $\left(v_{1}, v_{2}\right)=\left(v_{1}^{(1)}, v_{2}^{(1)}\right)$, since $v_{1}^{(1)}$ is a relative minimum, by Lemma 20 .

Since the smooth bounded solution constructed in any region must reach a maximum value of $v_{1}=|p(x, t)|^{2}$, and $v_{1}=v_{1}^{(4)}$ is the only maximum possible, the closure of the connected region where $\rho_{+}>0$ and $\rho_{-}>0$, to the left of $\left(v_{1}, v_{2}\right)=\left(v_{1}^{(4)}, v_{2}^{(4)}\right)$ and bounded by the algebraic set of Eq. (49), must be the unique compact connected region consisting of all the permissible initial conditions of $\left(v_{1}, v_{2}\right)$. When $s_{3} \leq s_{1}+s_{2}$, the minimum value of $v_{1}$ on this compact connected region is $v_{1}=0$, since all the nonzero critical points (other than the maximum) are saddle points, by Lemmas 19 and 20. When $s_{3}>s_{1}+s_{2}$, Lemma 24 shows that the minimum value of $v_{1}$ cannot be zero. Consequently, when $s_{3}>$ $s_{1}+s_{2}$, Lemmas 18,19 , and 20 show that the minimum of $v_{1}$ occurs at $\left(v_{1}, v_{2}\right)=\left(v_{1}^{(1)}, v_{2}^{(1)}\right)$.

This compact connected region in the $v_{1}-v_{2}$-plane parametrizes the permissible initial conditions for $\mu_{1}$ and $\mu_{2}$ through the explicit expressions of the first and second symmetric-polynomial constraints given in Corollaries 3 and 4. All smooth two-phase solutions of the NLS equation (1) are bounded, and so they must achieve a minimum and a maximum value of $v_{1}=|p(x, t)|^{2}$. Therefore the square of the modulus of any solution on the region of permissible values must oscillate between the maximum value $v_{1}=\left(s_{1}+s_{2}+s_{3}\right)^{2}$ and the minimum value of either $v_{1}=$ $\left(s_{1}+s_{2}-s_{3}\right)^{2}$, when $s_{3}>s_{1}+s_{2}$, or $v_{1}=0$, when $s_{3} \leq s_{1}+s_{2}$.

The simple dependence of the minimum and the maximum of the modulus of the solution on the branch points of the Riemann surface is consistent with the limit of one-phase solutions, viz., $s_{1} \rightarrow 0, v_{1}^{(2)} \rightarrow v_{1}^{(1)}$ and $v_{1}^{(3)} \rightarrow v_{1}^{(4)}$, in which there exists a single bounded solution [1] with minimum squared modulus $v_{1}=\left(s_{2}-s_{3}\right)^{2}$ and a maximum squared modulus $v_{1}=\left(s_{2}+\right.$ $\left.s_{3}\right)^{2}$. Numerical simulations of genus-two solutions [3,32,33] are also consistent with the result. For higher-phase solutions, an analogous result would require the inclusion of higher-time flows in the integrable NLS hierarchy. The spatial and temporal flows of the scalar NLS equation are not sufficient to span the higherdimensional torus of three-phase or higher-phase solutions. In general, the sum of the imaginary parts of the branch points of the higher-genus Riemann surface is expected to be an upper bound for the maximum modulus of the solution.

\section{Two-phase solutions of the NLS equation}

In this section, the smooth two-phase solution is constructed for the NLS equation (1). The Dirichlet eigenvalues of the solution were shown, for each fixed choice of the real curve $\mathscr{K}_{2}$ with three pairs of complex-conjugate branch points, to be a single compact connected two-dimensional manifold. As is well-known, the Dubrovin equations for the Dirichlet eigenvalues linearize via the Abel map onto the Jacobi variety of $\mathscr{K}_{2}$,

$$
\begin{aligned}
4 i t+d_{1} & =\int_{\lambda_{1}}^{\mu_{1}} \frac{d \mu_{1}}{\sqrt{\mathscr{R}\left(\mu_{1}\right)}}+\int_{\lambda_{3}}^{\mu_{2}} \frac{d \mu_{2}}{\sqrt{\mathscr{R}\left(\mu_{2}\right)}}, \\
2 i x-4 i c_{2} t+d_{2} & =\int_{\lambda_{1}}^{\mu_{1}} \frac{\mu_{1} d \mu_{1}}{\sqrt{\mathscr{R}\left(\mu_{1}\right)}}+\int_{\lambda_{3}}^{\mu_{2}} \frac{\mu_{2} d \mu_{2}}{\sqrt{\mathscr{R}\left(\mu_{2}\right)}},
\end{aligned}
$$

where

$d_{1}=\int_{\lambda_{1}}^{\mu_{10}} \frac{d \mu_{1}}{\sqrt{\mathscr{R}\left(\mu_{1}\right)}}+\int_{\lambda_{3}}^{\mu_{20}} \frac{d \mu_{2}}{\sqrt{\mathscr{R}\left(\mu_{2}\right)}}$,
$d_{2}=\int_{\lambda_{1}}^{\mu_{10}} \frac{\mu_{1} d \mu_{1}}{\sqrt{\mathscr{R}\left(\mu_{1}\right)}}+\int_{\lambda_{3}}^{\mu_{20}} \frac{\mu_{2} d \mu_{2}}{\sqrt{\mathscr{R}\left(\mu_{2}\right)}}$,

where the constants of integration $d_{1}$ and $d_{2}$ are not arbitrary but are determined by an allowed pair of initial values $\mu_{10}$ and $\mu_{20}$ which satisfy, along with the initial values of the real parameters $v_{1}$ and $v_{2}$, the algebraic constraints imposed by the invariant algebraic curve. Moreover, this set of permissible values for $d_{1}$ and $d_{2}$ is partitioned into equivalence classes lying on distinct non-intersecting two-real-dimensional planes in $\mathbb{C}^{2}$ formed by translation along the linear flows of Eq. (110) on the Jacobi variety of $\mathscr{K}_{2}$.

Solving Eqs. (110) for $\mu_{1}$ and $\mu_{2}$ is a special case of the Jacobi inversion problem. The Appendix contains the details of a classical method, based on the approach of Baker [27], of solving the Jacobi inversion problem in terms of the Kleinian elliptic functions $\sigma$ and $\zeta$ defined on the genus-two Riemann surface $\mathscr{K}_{2}$. A particular choice is made for the labeling of the branch points, viz., $\lambda_{1}=$ $\lambda_{2}^{*}=r_{1}+i s_{1}, \lambda_{3}=\lambda_{4}^{*}=r_{2}+i s_{2}$, and $\lambda_{5}=\lambda_{6}^{*}=r_{3}+i s_{3}$, where $r_{1} \leq r_{2} \leq r_{3}$, so that the canonical cycles are conveniently placed as in Fig. 7 in the Appendix. The formulas in Lemma 18 for the initial values of the Dirichlet eigenvalues will be used, since these formulas are symmetric with respect to the ordering of the real and imaginary parts of the branch points.

In particular, if $u^{\prime}=\left(u_{1}^{\prime}, u_{2}^{\prime}\right)^{T}$, with

$$
\begin{aligned}
u_{1}^{\prime} & =\int_{\lambda_{1}}^{\mu_{1}} \frac{d \mu_{1}}{\sqrt{\mathscr{R}\left(\mu_{1}\right)}}+\int_{\lambda_{3}}^{\mu_{2}} \frac{d \mu_{2}}{\sqrt{\mathscr{R}\left(\mu_{2}\right)}} \\
& =4 i t+d_{1}, \\
u_{2}^{\prime} & =\int_{\lambda_{1}}^{\mu_{1}} \frac{\mu_{1} d \mu_{1}}{\sqrt{\mathscr{R}\left(\mu_{1}\right)}}+\int_{\lambda_{3}}^{\mu_{2}} \frac{\mu_{2} d \mu_{2}}{\sqrt{\mathscr{R}\left(\mu_{2}\right)}} \\
& =2 i x+2 i \Lambda_{1} t+d_{2},
\end{aligned}
$$

so that

$u^{\prime}=i V x+i W t+d$

where

$$
\begin{aligned}
V & =(0,2)^{T}, \\
W & =\left(4,2 \Lambda_{1}\right)^{T}, \\
d & =\left(d_{1}, d_{2}\right)^{T},
\end{aligned}
$$

and $d_{1}$ and $d_{2}$ are defined in Eq. (111), then Eq. (A.64) produces simple formulas for the symmetric polynomials of $\mu_{1}$ and $\mu_{2}$,

$$
\begin{aligned}
\mu_{1}+\mu_{2}= & \frac{1}{2} \Lambda_{1}-\delta_{2}+\frac{\partial}{\partial u_{2}} \ln \left(\frac{\sigma\left(u^{\prime}+\int_{\lambda_{6}}^{\infty^{+}} d u\right)}{\sigma\left(u^{\prime}+\int_{\lambda_{6}}^{\infty^{-}} d u\right)}\right) \\
\mu_{1} \mu_{2}= & -\frac{1}{8} \Lambda_{1}^{2}+\frac{1}{2} \Lambda_{2}+\delta_{1}-\frac{\partial}{\partial u_{1}} \\
& \times \ln \left(\frac{\sigma\left(u^{\prime}+\int_{\lambda_{6}}^{\infty^{+}} d u\right)}{\sigma\left(u^{\prime}+\int_{\lambda_{6}}^{\infty^{-}} d u\right)}\right)
\end{aligned}
$$

where $\delta_{1}$ and $\delta_{2}$ are constants of integration expressible in terms of functions of the branch points. The solution $p$ to the NLS equation 
(1) is obtained from the trace formulas in Eqs. (14) and (115), viz.,

$$
\begin{aligned}
\frac{\partial}{\partial x} \ln p= & 2 i\left(\mu_{1}+\mu_{2}\right)-i \Lambda_{1} \\
= & 2 i \frac{\partial}{\partial u_{2}} \ln \left(\frac{\sigma\left(u^{\prime}+\int_{\lambda_{6}}^{\infty^{+}} d u\right)}{\sigma\left(u^{\prime}+\int_{\lambda_{6}}^{\infty^{-}} d u\right)}\right)-2 i \delta_{2} \\
\frac{\partial}{\partial t} \ln p= & \frac{\partial}{\partial x} \ln \left(\frac{\sigma\left(u^{\prime}+\int_{\lambda_{6}}^{\infty^{+}} d u\right)}{\sigma\left(u^{\prime}+\int_{\lambda_{6}}^{\infty^{-}} d u\right)}\right)-2 i \delta_{2}, \\
= & 4 i \frac{\partial}{\partial u_{1}} \ln \left(\frac{\sigma\left(u^{\prime}+\int_{\lambda_{6}}^{\infty^{+}} d u\right)}{\sigma\left(u^{\prime}+\int_{\lambda_{6}}^{\infty^{-}} d u\right)}\right)+2 i \Lambda_{1} \frac{\partial}{\partial u_{2}} \\
& \times \ln \left(\frac{\sigma\left(u_{2}-\frac{1}{2} i \Lambda_{1}^{2}+\int_{\lambda_{6}}^{\infty^{+}} d u\right)}{\sigma\left(u^{\prime}+\int_{\lambda_{6}}^{\infty^{-}} d u\right)} \ln p\right. \\
= & \frac{\partial}{\partial t} \ln \left(\frac{\sigma\left(u^{\prime}+\int_{\lambda_{6}}^{\infty^{+}} d u\right)}{\sigma\left(u^{\prime}+\int_{\lambda_{6}}^{\infty^{-}} d u\right)}\right)-4 i \delta_{1}-2 i \delta_{2} \Lambda_{1} .
\end{aligned}
$$

Hence, the two-phase solution to the NLS equation (1) is

$p(x, t)=\sqrt{v_{1}(0,0)} e^{i \phi(x, t)} \frac{\sigma\left(\beta^{-}\right)}{\sigma\left(\beta^{+}\right)} \frac{\sigma\left(i V x+i W t+\beta^{+}\right)}{\sigma\left(i V x+i W t+\beta^{-}\right)}$

where

$$
\begin{aligned}
\beta^{+} & =\int_{\lambda_{1}}^{\mu_{10}} d u+\int_{\lambda_{3}}^{\mu_{20}} d u+\int_{\lambda_{6}}^{\infty^{+}} d u, \\
\beta^{-} & =\int_{\lambda_{1}}^{\mu_{10}} d u+\int_{\lambda_{3}}^{\mu_{20}} d u+\int_{\lambda_{6}}^{\infty^{-}} d u, \\
\phi(x, t) & =-2 \delta_{2} x-\left(4 \delta_{1}+2 \delta_{2} \Lambda_{1}\right) t+\phi_{0},
\end{aligned}
$$

$\delta_{1}, \delta_{2} \in \mathbb{C}$ are given explicitly by Eq. (A.66), and $\phi_{0} \in \mathbb{R}$ is an arbitrary real constant. Note that $\delta_{1}$ and $\delta_{2}$ in $\phi$ are, in general, complex because there is also a complex phase in the ratio of the $\sigma$ functions. These complex phases cancel out, so that the solution remains bounded for all $x$ and $t$. The values of $v_{1}(0,0)>0$ and $\left(\mu_{10}, s_{10}\right),\left(\mu_{20}, s_{20}\right) \in \mathscr{K}_{2}$ can be explicitly given in terms of the branch points of the curve $\mathscr{K}_{2}$ by the formulas derived in the previous section.

It is convenient to remove the exponential factors from the $\sigma$ functions in Eq. (117) and write the two-phase solution in terms of Riemann theta functions. Using the definition of the theta function given in the Appendix, the solution in Eq. (117) is

$$
\begin{aligned}
p(x, t)= & \sqrt{v_{1}(0,0)} e^{i \xi(x, t)} \frac{\theta\left(\frac{1}{2} \omega^{-1} \beta^{-}\right)}{\theta\left(\frac{1}{2} \omega^{-1} \beta^{+}\right)} \\
& \times \frac{\theta\left(\frac{1}{2} \omega^{-1}\left(i V x+i W t+\beta^{+}\right)\right)}{\theta\left(\frac{1}{2} \omega^{-1}\left(i V x+i W t+\beta^{-}\right)\right)},
\end{aligned}
$$

where

$$
\begin{aligned}
\xi(x, t)= & \phi(x, t)+\left(2 \alpha_{11} 4 t+\left(\alpha_{12}+\alpha_{21}\right)\left(2 x+2 \Lambda_{1} t\right)\right) \int_{\infty^{-}}^{\infty^{+}} d u_{1} \\
& +\left(2 \alpha_{22}\left(2 x+2 \Lambda_{1} t\right)+\left(\alpha_{12}+\alpha_{21}\right) 4 t\right) \int_{\infty^{-}}^{\infty^{+}} d u_{2} .
\end{aligned}
$$

Lemma 25. The exponential factor $e^{i \xi(x, t)}$ in Eq. (119) is a phase factor of modulus 1 , viz.,

$\xi(x, t)=\kappa_{1} x+\kappa_{2} t+\phi_{0}$,

where the wavenumbers $\kappa_{1}, \kappa_{2} \in \mathbb{R}$ are given by

$$
\begin{aligned}
\kappa_{1}= & 2\left(\lambda_{1}+\lambda_{1}^{*}-\frac{1}{2} \Lambda_{1}\right)-\left(\omega^{-1}\right)_{12}\left(\frac{\theta_{1}^{+}}{\theta^{+}}-\frac{\theta_{1}^{-}}{\theta^{-}}\right)-\left(\omega^{-1}\right)_{22} \\
& \times\left(\frac{\theta_{2}^{+}}{\theta^{+}}-\frac{\theta_{2}^{-}}{\theta^{-}}\right), \\
\kappa_{2}= & 4\left(-\left|\lambda_{1}\right|^{2}-\frac{1}{8} \Lambda_{1}^{2}+\frac{1}{2} \Lambda_{2}\right)-2\left(\omega^{-1}\right)_{11}\left(\frac{\theta_{1}^{+}}{\theta^{+}}-\frac{\theta_{1}^{-}}{\theta^{-}}\right)(1 \\
& -2\left(\omega^{-1}\right)_{21}\left(\frac{\theta_{2}^{+}}{\theta^{+}}-\frac{\theta_{2}^{-}}{\theta^{-}}\right)+\Lambda_{1}\left(2\left(\lambda_{1}+\lambda_{1}^{*}-\frac{1}{2} \Lambda_{1}\right)\right. \\
& \left.-\left(\omega^{-1}\right)_{12}\left(\frac{\theta_{1}^{+}}{\theta^{+}}-\frac{\theta_{1}^{-}}{\theta^{-}}\right)-\left(\omega^{-1}\right)_{22}\left(\frac{\theta_{2}^{+}}{\theta^{+}}-\frac{\theta_{2}^{-}}{\theta^{-}}\right)\right),
\end{aligned}
$$

and $\phi_{0} \in \mathbb{R}$ is an arbitrary phase.

Proof. Since $\omega^{-1}$ is purely imaginary (see the Appendix), it is sufficient to show that the differences of logarithmic derivatives of $\theta$ of the form, for $j=1,2$,

$\frac{\theta_{j}^{+}}{\theta^{+}}-\frac{\theta_{j}^{-}}{\theta^{-}}$

are also purely imaginary. Consider the complex conjugate

$$
\begin{aligned}
\left(\frac{1}{2} \omega^{-1}\left(\int_{\lambda_{6}}^{\infty^{+}} d u+\int_{\lambda_{3}}^{\lambda_{2}} d u\right)\right)^{*} \\
=-\frac{1}{2} \omega^{-1}\left(\int_{\lambda_{5}}^{\infty^{+}} d u+\int_{\lambda_{4}}^{\lambda_{1}} d u\right) \\
=-\frac{1}{2} \omega^{-1}\left(\int_{\lambda_{6}}^{\infty^{+}} d u+\int_{\lambda_{3}}^{\lambda_{2}} d u\right) \\
\quad-v^{\lambda_{6}, \lambda_{5}}-v^{\lambda_{1}, \lambda_{2}}-v^{\lambda_{3}, \lambda_{4}} \\
=-\frac{1}{2} \omega^{-1}\left(\int_{\lambda_{6}}^{\infty^{+}} d u+\int_{\lambda_{3}}^{\lambda_{2}} d u\right)+m+\tau m^{\prime},
\end{aligned}
$$

where the sum of the half-period characteristics is zero, i.e., an integer lattice translation for some $m, m^{\prime} \in \mathbb{Z}^{2}$. Similarly,

$$
\begin{aligned}
& \left(\frac{1}{2} \omega^{-1}\left(\int_{\lambda_{6}}^{\infty^{-}} d u+\int_{\lambda_{3}}^{\lambda_{2}} d u\right)\right)^{*} \\
& =-\frac{1}{2} \omega^{-1}\left(\int_{\lambda_{6}}^{\infty^{-}} d u+\int_{\lambda_{3}}^{\lambda_{2}} d u\right)+m+\tau m^{\prime},
\end{aligned}
$$

with the same $m, m^{\prime} \in \mathbb{Z}^{2}$ as previously, since we can integrate along the same paths in the calculation of the half-integer characteristics.

Now using the fact that $\theta$ is an even function and the partial derivatives $\theta_{1}$ and $\theta_{2}$ are odd functions, and the transformation property of the logarithmic derivatives in Eq. (A.29), we obtain, for $j=1,2$,

$$
\begin{aligned}
& \left(\frac{\theta_{j}^{+}}{\theta^{+}}\right)^{*}=-2 \pi i m_{j}^{\prime}-\frac{\theta_{j}^{+}}{\theta^{+}}, \\
& \left(\frac{\theta_{j}^{-}}{\theta^{-}}\right)^{*}=-2 \pi i m_{j}^{\prime}-\frac{\theta_{j}^{-}}{\theta^{-}} .
\end{aligned}
$$


Hence

$$
\left(\frac{\theta_{j}^{+}}{\theta^{+}}-\frac{\theta_{j}^{-}}{\theta^{-}}\right)^{*}=-\left(\frac{\theta_{j}^{+}}{\theta^{+}}-\frac{\theta_{j}^{-}}{\theta^{-}}\right) .
$$

Finally, all the previous results can be summarized in the following theorem, in which the solution of the NLS equation (1) is explicitly constructed in terms of the branch points of the Riemann surface $\mathscr{K}_{2}$ and the correct initial values for the Dirichlet eigenvalues, so that all reality conditions are satisfied. Moreover simple formulas for the minimum and the maximum of the modulus of the solution are determined from Theorem 7, based solely on the imaginary parts of the branch points of $\mathscr{K}_{2}$.

Theorem 8. Each smooth two-phase solution of the focusing NLS equation (1) is a two-real-dimensional torus submanifold, modulo a circle of complex phase factors, of the Jacobi variety of a nonsingular genus-two Riemann surface $\mathscr{K}_{2}$, given by Eq. (22), with branch points $\lambda_{1}=\lambda_{2}^{*}=r_{1}+i s_{1}, \lambda_{3}=\lambda_{4}^{*}=r_{2}+i s_{2}, \lambda_{5}=\lambda_{6}^{*}=r_{3}+i s_{3}$, where $r_{1}, r_{2}, r_{3} \in \mathbb{R}$ and $s_{1}, s_{2}, s_{3}>0$, as labeled in Fig. 7. The solution can be written as a ratio of theta functions associated with $\mathscr{K}_{2}$, as defined in the Appendix, viz.,

$$
\begin{aligned}
p(x, t)= & \left(s_{1}+s_{2}+s_{3}\right) e^{i \xi\left(x-x_{0}, t-t_{0}\right)} \frac{\theta\left(\frac{1}{2} \omega^{-1} \beta^{-}\right)}{\theta\left(\frac{1}{2} \omega^{-1} \beta^{+}\right)} \\
& \times \frac{\theta\left(\frac{1}{2} \omega^{-1} U^{+}(x, t)\right)}{\theta\left(\frac{1}{2} \omega^{-1} U^{-}(x, t)\right)},
\end{aligned}
$$

where

$U^{+}(x, t)=i V\left(x-x_{0}\right)+i W\left(t-t_{0}\right)+\beta^{+}$,

$U^{-}(x, t)=i V\left(x-x_{0}\right)+i W\left(t-t_{0}\right)+\beta^{-}$,

with $V=(0,2)^{T}, W=\left(4,4\left(r_{1}+r_{2}+r_{3}\right)\right)^{T}$,

$$
\begin{aligned}
\beta^{+}= & \int_{\lambda_{1}}^{\left(\mu_{10},-\sqrt{\mathscr{R}\left(\mu_{10}\right)}\right)} d u+\int_{\lambda_{3}}^{\left(\mu_{20}, \sqrt{\mathscr{R}\left(\mu_{20}\right)}\right)} d u \\
& +\int_{\lambda_{6}}^{\infty^{+}} d u, \\
\beta^{-}= & \int_{\lambda_{1}}^{\left(\mu_{10},-\sqrt{\mathscr{R}\left(\mu_{10}\right)}\right)} d u+\int_{\lambda_{3}}^{\left(\mu_{20}, \sqrt{\mathscr{R}\left(\mu_{20}\right)}\right)} d u \\
& +\int_{\lambda_{6}}^{\infty^{-}} d u,
\end{aligned}
$$

where the initial values of the Dirichlet eigenvalues $\left(\mu_{10}\right.$, $\left.-\sqrt{\mathscr{R}\left(\mu_{10}\right)}\right),\left(\mu_{20}, \sqrt{\mathscr{R}\left(\mu_{20}\right)}\right) \in \mathscr{K}_{2}$ are given by the symmetric expressions in Eqs. (71) and (72) of Lemma 18, the phase $\xi(x, t)$ is given by $E q$. (121), and $\left(x_{0}, t_{0}\right) \in \mathbb{R}^{2}$ is an arbitrary location for the maximum modulus of the solution. Moreover, if the imaginary parts of the branch points are re-labeled, if necessary, so that $0<s_{1} \leq s_{2} \leq s_{3}$, then the two-phase solution given by Eq. (128) has maximum modulus $s_{1}+s_{2}+s_{3}>0$ and minimum modulus $s_{3}-s_{1}-s_{2}>0$, if $s_{3}>s_{1}+s_{2}$, or zero, if $s_{3} \leq s_{1}+s_{2}$.

\section{Example solutions}

Two solutions are constructed using the formula of Theorem 8 . Both solutions are constructed using Maple and a simple integration algorithm, based on Simpson's rule, to compute the necessary contour integrals on the Riemann surface $\mathscr{K}_{2}$. For integrals involving points over infinity or branch points, Maple's algcurve package is used to compute a Puiseux expansion near the point of interest, which is integrated directly using Maple's int command [34].

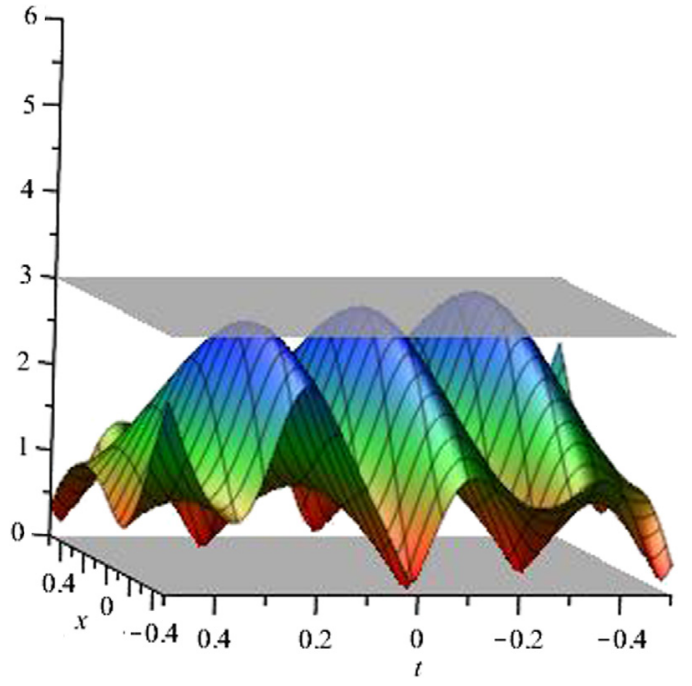

Fig. 1. Modulus of a solution of the NLS equation (1) with minimum 0 and maximum 3 for $(x, t) \in[-0.5,0.5] \times[-0.5,0.5]$, on a $100 \times 100$ grid. Transparent horizontal planes have been added to the graph at the height of the minimum and the maximum. The six branch points have imaginary parts with absolute values equal to $s_{1}=1, s_{2}=1$, and $s_{3}=1$.

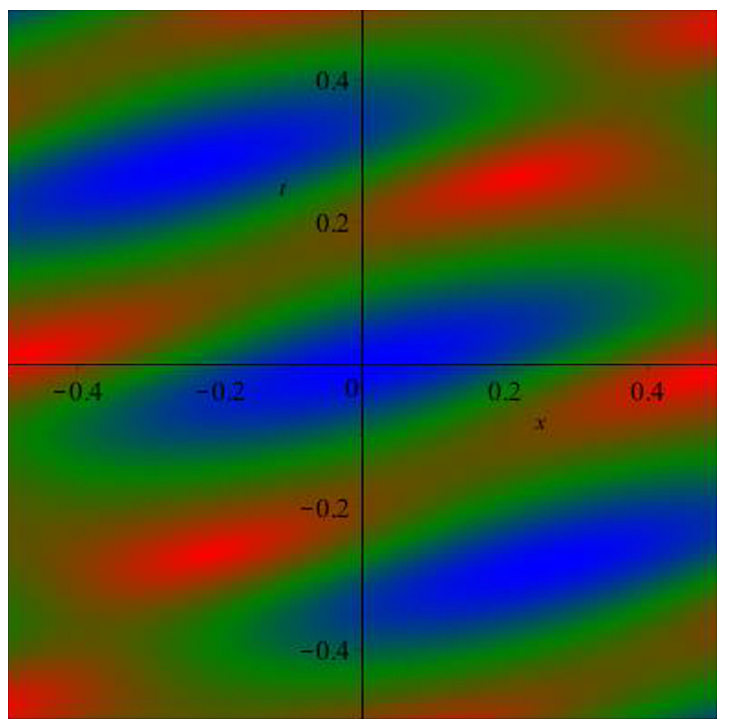

Fig. 2. Density plot of the same solution of the NLS equation (1) as in Fig. 1. The height of the function is indicated by the colors red, green, and blue scaled to the interval $[0,3]$. (For interpretation of the references to colour in this figure legend the reader is referred to the web version of this article.)

In Figs. 1, 2, and 3, the modulus of the same solution of the NLS equation (1) is constructed using Eq. (128). The solution is graphed using three different methods: a three-dimensional plot, a density plot, and a plot of the list of values for the modulus. The branch points are chosen to be

$-2+i,-2-i,-1+i,-1-i, 1+i, 1-i$,

so that the imaginary parts of the branch points are all equal, viz., $s_{1}=1, s_{2}=1$, and $s_{3}=1$. According to Theorem 8 , the maximum modulus is $s_{1}+s_{2}+s_{3}=3$, and the minimum modulus is 0 because $s_{3}<s_{1}+s_{2}$.

In Figs. 4, 5, and 6, the modulus of a second solution is constructed using Eq. (128). In this case the branch points are chosen to be

$-2+3 i,-2-3 i,-1+i,-1-i, 1+i, 1-i$, 


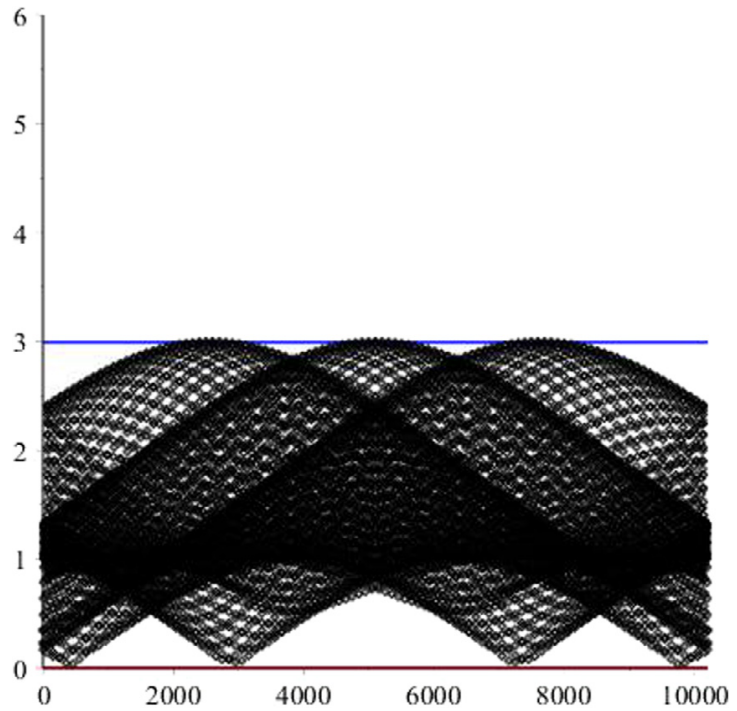

Fig. 3. Plot of the list of values of the modulus of the solution of the NLS equation (1) in Figs. 1 and 2. The values in the list are ordered by successive cross-sections of constant $x$ of the surface in Fig. 1. Four occurrences of the minimum and three occurrences of the maximum can be clearly seen.

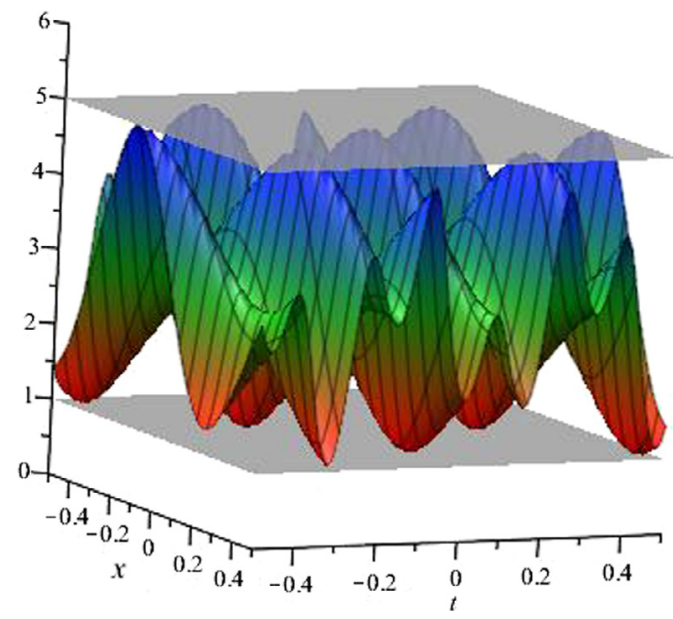

Fig. 4. Modulus of a solution of the NLS equation (1) with minimum 1 and maximum 5 for $(x, t) \in[-0.5,0.5] \times[-0.5,0.5]$, on a $100 \times 100$ grid. Transparent horizontal planes have been added to the graph at the height of the minimum and the maximum. The six branch points have imaginary parts with absolute values, ordered from least to greatest, equal to $s_{1}=1, s_{2}=1$, and $s_{3}=3$.

so that the imaginary parts of the branch points, in increasing order, are $s_{1}=1, s_{2}=1$, and $s_{3}=3$. Thus the maximum modulus is $s_{1}+s_{2}+s_{3}=5$, and the minimum modulus is $s_{3}-s_{1}-s_{2}=1$, since $s_{3}>s_{1}+s_{2}$. Recall that in Theorem 6 the labeling of the three imaginary parts is chosen so that $0<s_{1} \leq s_{2} \leq s_{3}$, and that the minimum modulus and the maximum modulus are independent of the (possibly different) labeling of the branch points shown in Fig. 7 and used in the explicit construction of the solution.

\section{Conclusion}

Simple formulas, in terms of the imaginary parts of the branch points, have been found for the minimum modulus and the maximum modulus of each smooth two-phase solution of the scalar focusing cubic NLS equation (1). The solution of the NLS equation is written as a ratio of theta functions associated with the Riemann surface of the invariant spectral curve. The theta function formula for the two-phase solution is new in the following sense: all the parameters in the solution are written explicitly in terms

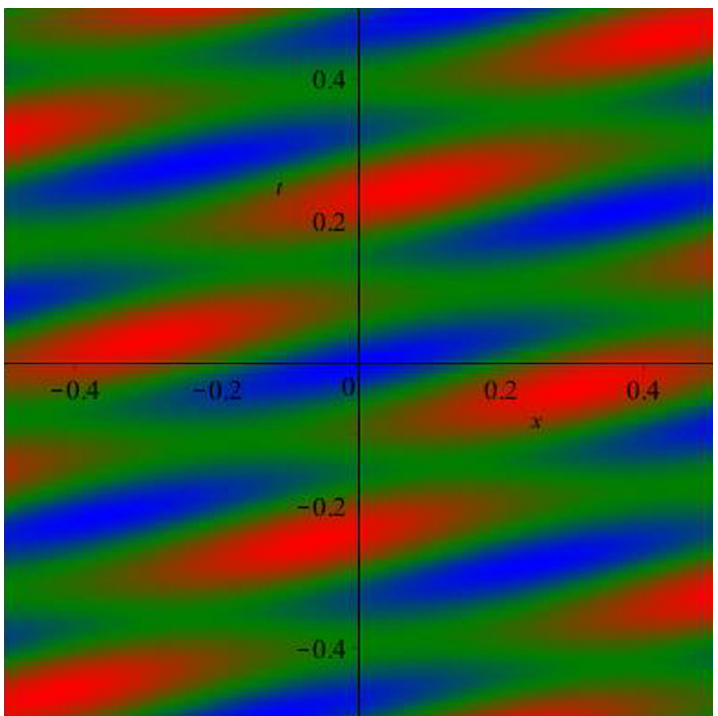

Fig. 5. Density plot of the same solution of the NLS equation (1) as in Fig. 4. The height of the function is indicated by the colors red, green, and blue scaled to the interval $[1,5]$. (For interpretation of the references to colour in this figure legend, the reader is referred to the web version of this article.)

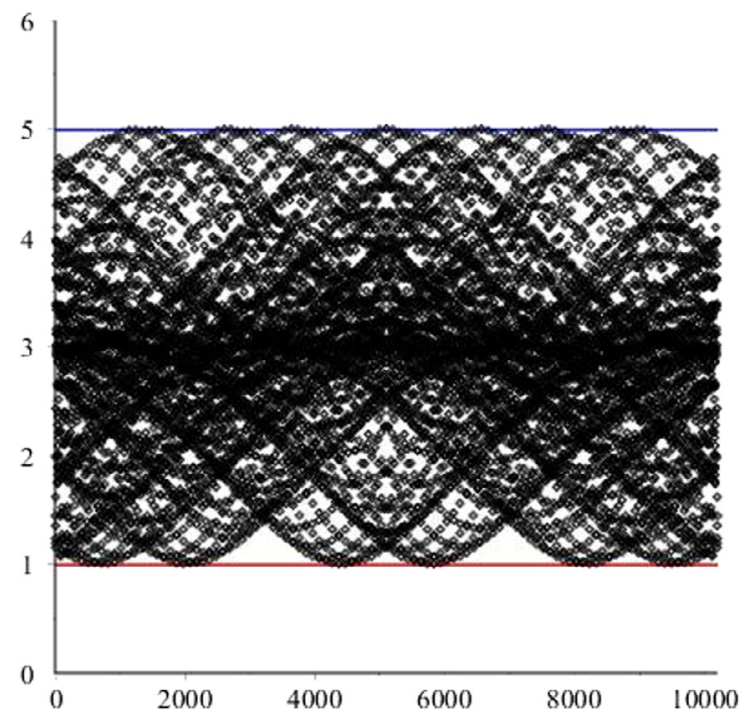

Fig. 6. Plot of the list of values of the modulus of the solution of the NLS equation (1) in Figs. 4 and 5. The values in the list are ordered by successive cross-sections of constant $x$ of the surface in Fig. 4. Six occurrences of the minimum and seven occurrences of the maximum can be clearly seen.

of the branch points of the genus-two Riemann surface and the initial values of the Dirichlet eigenvalues which satisfy the reality conditions. The Appendix provides the details of the solution of the Jacobi inversion problem as it applies to the real genus-two Riemann surface of the integrable NLS equation (1).

The simple dependence of the minimum and the maximum amplitudes of the two-phase solution on the imaginary parts of the branch points is consistent with known results for zero-phase and one-phase solutions [1] and with numerical simulations of twophase solutions $[3,32,33]$. For higher-phase solutions of the scalar cubic NLS equation (1), the two-real-dimensional linear flow on the Jacobi variety will not span the higher-dimensional real torus of the solution, as it does in the two-dimensional case, so the sum of the imaginary parts of the branch points is expected to be only an upper bound on the modulus of the quasi-periodic solution.

The representation obtained in this manuscript may be useful in characterizing modulations of two-phase solutions, for example, 


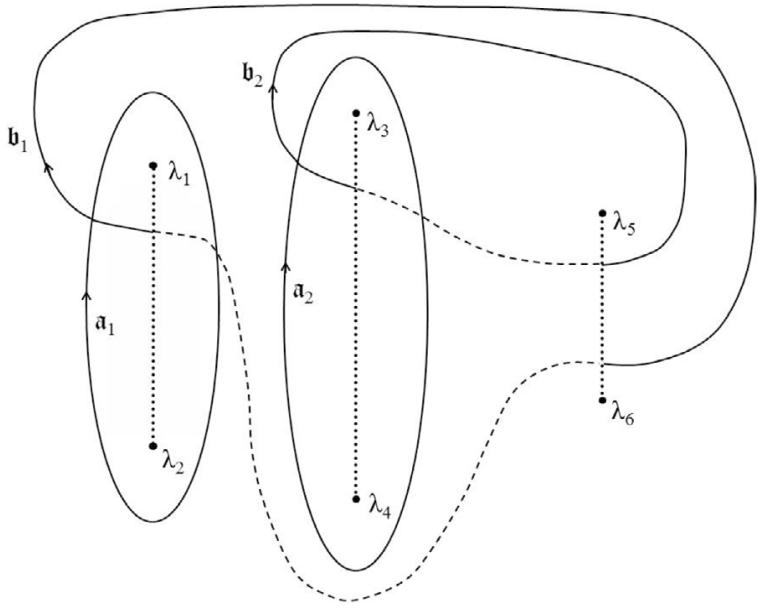

Fig. 7. Basis of homology cycles for a two-phase solution of the NLS equation (1).

in the vicinity of a gradient catastrophe in which spikes are formed which are limits of two-phase solutions [2,3]. More work is needed to extend the current results to the scalar defocusing cubic NLS equation and the Manakov system of coupled NLS equations [7,8, $35,36]$.

It is well-known [9-14,18,20,22,23] how to obtain the solution of the NLS equation in terms of theta functions associated with the Riemann surface of an invariant spectral curve. However, in previous studies, the reality conditions on the initial values of the Dirichlet eigenvalues are either satisfied in the sense of an existence result or satisfied at the level of quantities defined on the Jacobi variety of the Riemann surface. In this paper, an alternative effective integration is implemented, analogous to the results for elliptic solutions obtained by Kamchatnov [1,24]. Using the explicit solution method for a quartic polynomial equation satisfied by the Dirichlet eigenvalues and the explicit solution of the Jacobi inversion problem for a genus-two Riemann surface, the reality conditions on the integration constants are explicitly satisfied in terms of the physically-meaningful modulus and wavenumber of the solution. Consequently, a new effective theta function representation of the solution is constructed in which the parameters in the solution are explicitly constructed from the branch points of the Riemann surface and the real initial values of the Dirichlet eigenvalues. Moreover, simple new formulas for the minimum modulus and the maximum modulus of the solution are found in terms of the imaginary parts of the branch points.

\section{Appendix. The Jacobi inversion problem}

The inversion of the Abelian integrals in Eq. (29) for $\mu_{1}=$ $\mu_{1}(x, t)$ and $\mu_{2}=\mu_{2}(x, t)$ is called the Jacobi inversion problem. Classically [27] the inversion problem for the symmetric polynomials $\mu_{1}+\mu_{2}$ and $\mu_{1} \mu_{2}$ was solved in terms of the Kleinian sigma and zeta functions, defined on a genus-two Riemann surface corresponding to a canonical polynomial of degree five, which generalize the more familiar elliptic functions, i.e., the Weierstrass sigma and zeta functions. A more modern treatment [28] shows how the Kleinian elliptic functions can be used to solve the inversion problem for hyperelliptic Riemann surfaces corresponding to even-degree polynomials, such as $\mathscr{K}_{2}$. Consequently, some new details are presented in the Appendix that are necessary to calculate all the required quantities in the explicit construction of the two-phase solution of the focusing NLS equation (1), whose invariant polynomial is of degree six in the spectral parameter.

\section{A.1. Differential identities on the Riemann surface}

In order to solve the Jacobi inversion problem (29), several definitions and fundamental identities involving differentials on the Riemann surface are needed. The goal of the present manuscript is to keep technicalities to a minimum and follow as closely as possible the classical approach of Baker [27], while using the more modern language found in [9,28]. In general, most of the proofs of the statements in the Appendix are found in [27] or [28] and are omitted from the Appendix. However, in some cases proofs are sketched in order to clarify how the result works in this specific application.

It is assumed that $\mathscr{K}_{2}$ is a nonsingular genus-two Riemann surface satisfying the reality condition, viz., there are six distinct branch points forming three complex-conjugate pairs. A canonical basis of homology cycles, $\left\{\mathfrak{a}_{1}, \mathfrak{a}_{2} ; \mathfrak{b}_{1}, \mathfrak{b}_{2}\right\}$, is chosen to have the following intersection properties $\mathfrak{a}_{1} \circ \mathfrak{a}_{2}=\mathfrak{b}_{1} \circ \mathfrak{b}_{2}=0$ and $\mathfrak{a}_{1} \circ \mathfrak{b}_{1}=\mathfrak{a}_{2} \circ \mathfrak{b}_{2}=1$, as shown in Fig. 7. In particular, it is convenient to choose the ordering of the branch points, without loss of generality, so that the real parts are labeled in increasing order, $r_{1} \leq r_{2} \leq r_{3}$, where $\lambda_{1}=\lambda_{2}^{*}=r_{1}+i s_{1}, \lambda_{3}=\lambda_{4}^{*}=r_{2}+i s_{2}$, and $\lambda_{5}=\lambda_{6}^{*}=r_{3}+i s_{3}$, and $s_{1}, s_{2}, s_{3}>0$. Recall that this is a different convention than was used to label the branch points in Theorem 6, where the imaginary parts, not the real parts, were labeled in increasing order.

The action of the natural hyperelliptic involution $\iota$ on $\mathscr{K}_{2}$ is extended in a natural way to the homology cycles, and the cycles are chosen such that

$\iota\left(\mathfrak{a}_{1}\right)=-\mathfrak{a}_{1}$,

$\iota\left(\mathfrak{a}_{2}\right)=-\mathfrak{a}_{2}$,

$\iota\left(\mathfrak{b}_{1}\right)=-\mathfrak{b}_{1}$,

$\iota\left(\mathfrak{b}_{2}\right)=-\mathfrak{b}_{2}$.

In particular, the arrangement of cycles is chosen as shown in Fig. 7 for the case of three complex-conjugate pairs of branch points which satisfy the reality conditions for the NLS equation (1). Similarly, the natural action of the anti-holomorphic involution * on the cycles is

$*\left(\mathfrak{a}_{1}\right)=-\mathfrak{a}_{1}$,
$*\left(\mathfrak{a}_{2}\right)=-\mathfrak{a}_{2}$,
$*\left(\mathfrak{b}_{1}\right)=\mathfrak{b}_{1}+\mathfrak{a}_{2}$,
$*\left(\mathfrak{b}_{2}\right)=\mathfrak{b}_{2}+\mathfrak{a}_{1}$.

Now consider the two holomorphic integrals that appear in the Jacobi inversion problem (29),

$d u_{1}=\frac{d \lambda}{w}, \quad d u_{2}=\frac{\lambda d \lambda}{w}$.

The natural action of the anti-holomorphic involution on these two differentials is $* d u_{i}=d u_{i}^{*}$, for $i=1,2$, where $d u_{i}^{*}$ denotes the complex conjugate of the differential. The periods of the above differentials around the basis cycles are defined, for $i, j=1,2$, as

$\oint_{\mathfrak{a}_{j}} d u_{i}=2 \omega_{i j}, \quad \oint_{\mathfrak{b}_{j}} d u_{i}=2 \omega_{i j}^{\prime}$.

Notice that for the given choice of the basis cycles,

$\omega_{i j}^{*}=\oint_{\mathfrak{a}_{j}} d u_{i}^{*}=\oint_{\mathfrak{a}_{j}} * d u_{i}=\oint_{*\left(\mathfrak{a}_{j}\right)} d u_{i}=\oint_{-\mathfrak{a}_{j}} d u_{i}=-\omega_{i j}$,

so that $\Re\left(\omega_{i j}\right)=0$.

A normalized basis of holomorphic differentials, canonically dual to the basis of homology cycles, can now be constructed. Let, for $i=1,2$,

$d v_{i}=\sum_{j=1}^{2} \frac{1}{2}\left(\omega^{-1}\right)_{i j} d u_{j}$, 
then the periods are normalized in the sense that, for $i, j=1,2$,

$$
\oint_{\mathfrak{a}_{j}} d v_{i}=\delta_{i j}, \quad \oint_{\mathfrak{b}_{j}} d v_{i}=\tau_{i j},
$$

where $\delta_{i j}$ is the Kronecker delta symbol. A standard result, using Riemann's bilinear relations, is that the determinant of the matrix $\omega=\left(\omega_{i j}\right)$ is nonzero, and the matrix

$\tau=\omega^{-1} \omega^{\prime}$

is symmetric with a positive definite imaginary part.

With the particular choice of the canonical cycles shown in Fig. 7, the symmetry of $\mathscr{K}_{2}$ implies further information about the real part $\Re(\tau)$ of $\tau$, viz.,

$$
\begin{aligned}
\tau_{i j}^{*} & =\oint_{\mathfrak{b}_{j}} d v_{i}^{*} \\
& =\oint_{\mathfrak{b}_{j}} \sum_{k=1}^{2} \frac{1}{2}\left(\omega^{-1}\right)_{i k}^{*} d u_{k}^{*} \\
& =-\sum_{k=1}^{2} \frac{1}{2}\left(\omega^{-1}\right)_{i k} \oint_{\mathfrak{b}_{j}} * d u_{k} \\
& =-\sum_{k=1}^{2} \frac{1}{2}\left(\omega^{-1}\right)_{i k} \oint_{*\left(\mathfrak{b}_{j}\right)} d u_{k} \\
& =-\sum_{k=1}^{2} \frac{1}{2}\left(\omega^{-1}\right)_{i k} \oint_{\mathfrak{b}_{j}+\hat{a}_{j}} d u_{k} \\
& =-\sum_{k=1}^{2} \frac{1}{2}\left(\omega^{-1}\right)_{i k}\left(2 \omega_{k j}^{\prime}+2 \hat{\omega}_{k j}\right) \\
& =-\tau_{i j}-\hat{\delta}_{i j},
\end{aligned}
$$

where $\hat{\mathfrak{a}}_{1}=\mathfrak{a}_{2}$ and $\hat{\mathfrak{a}}_{2}=\mathfrak{a}_{1}$, so that the first and second columns of $\hat{\omega}$ are the same as the second and first columns, respectively, of $\omega$, and

$\hat{\delta}_{i j}=\left(\begin{array}{ll}0 & 1 \\ 1 & 0\end{array}\right)$.

Therefore

$\Re(\tau)=-\frac{1}{2}\left(\begin{array}{ll}0 & 1 \\ 1 & 0\end{array}\right)$.

We now introduce two differentials of the second kind associated with $d u_{1}$ and $d u_{2}$, see [27], viz.,

$d r_{1}=\frac{4 \lambda^{4}-3 \Lambda_{1} \lambda^{3}+2 \Lambda_{2} \lambda^{2}-\Lambda_{3} \lambda}{4 w} d \lambda$

$d r_{2}=\frac{2 \lambda^{3}-\Lambda_{1} \lambda^{2}}{4 w} d \lambda$

These differentials are holomorphic except for poles of the second kind at the points at infinity, and they satisfy the following identity of two-differentials,

$$
\begin{aligned}
& \frac{\partial}{\partial z} \frac{w+s}{\lambda-z} \frac{1}{2 w} d z d \lambda+d u_{1}(\lambda) d r_{1}(z)+d u_{2}(\lambda) d r_{2}(z) \\
& \quad=\frac{F(\lambda, z)+2 w s}{4(\lambda-z)^{2}} \frac{d \lambda}{w} \frac{d z}{s}
\end{aligned}
$$

where $w^{2}=\mathscr{R}(\lambda), s^{2}=\mathscr{R}(z)$, and the symmetric function $F(\lambda, z)=F(z, \lambda)$, where

$$
\begin{aligned}
F(\lambda, z)= & 2 \Lambda_{6}-\Lambda_{5}(\lambda+z)+\lambda z\left(2 \Lambda_{4}-\Lambda_{3}(\lambda+z)\right) \\
& +\lambda^{2} z^{2}\left(2 \Lambda_{2}-\Lambda_{1}(\lambda+z)\right)+2 \lambda^{3} z^{3} .
\end{aligned}
$$

The periods of the differentials $d r_{1}$ and $d r_{2}$ are defined, for $i, j=$ 1,2 , as

$$
\oint_{\mathfrak{a}_{j}} d r_{i}=-2 \eta_{i j}, \quad \oint_{\mathfrak{b}_{j}} d r_{i}=-2 \eta_{i j}^{\prime} \text {. }
$$

The periods satisfy the following relations,

$$
\begin{gathered}
\omega \omega^{\prime T}-\omega^{\prime} \omega^{T}=0, \\
\eta \eta^{\prime T}-\eta^{\prime} \eta^{T}=0, \\
\omega \eta^{\prime T}-\omega^{\prime} \eta^{T}=-\frac{\pi i}{2} \mathbf{I},
\end{gathered}
$$

where $\omega^{T}$ denotes the transpose of the matrix $\omega$, and $\mathbf{I}$ is the $2 \times 2$ identity matrix. Eq. (A.13) shows that the symmetric twodifferential on the right-hand side of the equation gives Klein's symmetric integral of the third kind,

$$
\mathscr{K}(\lambda, a ; z, b)=\int_{a}^{\lambda} \int_{b}^{z} \frac{F(\lambda, z)+2 w s}{4(\lambda-z)^{2}} \frac{d \lambda}{w} \frac{d z}{s},
$$

with logarithmic infinities of coefficients 1 and -1 respectively at $\lambda=z$ and $\lambda=b$. Notice that the symmetry of the integrand implies that $\mathscr{K}(\lambda, a ; z, b)=\mathscr{K}(z, b ; \lambda, a)$.

Definition 2. The normalized differential of the third kind having a simple pole of residue +1 at the location $\lambda=z$, simple pole with residue -1 at the location $\lambda=b$, and zero periods around the cycles $\mathfrak{a}_{1}$ and $\mathfrak{a}_{2}$ is denoted by $d \Pi_{z, b}$. The normalized differential of the second kind having a single pole of order two at $\lambda=z$ with coefficient 1 and zero periods around the cycles $\mathfrak{a}_{1}$ and $\mathfrak{a}_{2}$ is denoted by $d \Gamma_{z}$.

Notice that $d \Gamma_{z}$ can be obtained from $d \Pi_{z, b}$ by differentiation of the latter with respect to the parameter $z$. Thus an application of Riemann's bilinear relations using $d v_{i}, i=1,2$, and $d \Pi_{z, b}$ shows that

$\oint_{\mathfrak{b}_{1}} d \Gamma_{z}=2 \pi i \frac{d v_{1}}{d z}(z), \quad \oint_{\mathfrak{b}_{2}} d \Gamma_{z}=2 \pi i \frac{d v_{2}}{d z}(z)$.

Another application of Riemann's bilinear relations to $d \Pi_{\lambda, a}$ and $d \Pi_{z, b}$, demonstrates the following symmetry,

$\int_{a}^{\lambda} d \Pi_{z, b}=\int_{b}^{z} d \Pi_{\lambda, a}$.

Since $\mathscr{K}(\lambda, a ; z, b)$ is an integral of the third kind, it can be written in terms of the normalized integral of the third kind and two linearly independent integrals of the first kind, leading to the following definition, in which the symmetry of $\mathscr{K}$ and Eq. (A.19) are used.

Definition 3. Let $\alpha$ be the $2 \times 2$ symmetric matrix defined by the identity

$$
\begin{aligned}
\mathscr{K}(\lambda, a ; z, b)= & \int_{a}^{\lambda} d \Pi_{z, b}-2 \sum_{i=1}^{2} \sum_{j=1}^{2} \alpha_{i j} \\
& \times \int_{a}^{\lambda} d u_{i} \int_{b}^{z} d u_{j} .
\end{aligned}
$$

Differentiation of the above identity with respect to local parameters $\lambda$ and $z$ gives the following identity between twodifferentials,

$$
\begin{aligned}
\frac{F(\lambda, z)+2 w s}{4(\lambda-z)^{2}} \frac{d \lambda}{w} \frac{d z}{s}= & d \Gamma_{z}(\lambda) d z-2 \sum_{i=1}^{2} \sum_{j=1}^{2} \alpha_{i j} \\
& \times d u_{i}(\lambda) d u_{j}(z) .
\end{aligned}
$$


If the roles of $\lambda$ and $z$ are interchanged in Eq. (A.13), using the symmetry of the two-form on the right-hand side, then Eq. (A.21), can be re-written as

$$
\begin{aligned}
& d u_{1}(z) d r_{1}(\lambda)+d u_{2}(z) d r_{2}(\lambda)+2 \sum_{i=1}^{2} \sum_{j=1}^{2} \alpha_{i j} d u_{i}(\lambda) d u_{j}(z) \\
& \quad=d \Gamma_{z}(\lambda) d z-\frac{\partial}{\partial \lambda} \frac{s+w}{z-\lambda} \frac{d \lambda d z}{2 s} .
\end{aligned}
$$

Integration in $\lambda$ of Eq. (A.22) about the basis cycles $\mathfrak{a}_{k}$ and $\mathfrak{b}_{k}$, for $k=1,2$, gives two identities of the following differentials of $z$,

$$
\begin{aligned}
& -2 \eta_{1 k} d u_{1}-2 \eta_{2 k} d u_{2}+4 \sum_{i=1}^{2} \sum_{j=1}^{2} \alpha_{i j} \omega_{i k} d u_{j}=0, \\
& -2 \eta_{1 k}^{\prime} d u_{1}-2 \eta_{2 k}^{\prime} d u_{2}+4 \sum_{i=1}^{2} \sum_{j=1}^{2} \alpha_{i j} w_{i k}^{\prime} d u_{j}=2 \pi i d v_{k} .
\end{aligned}
$$

Since $d u_{1}$ and $d u_{2}$ are linearly independent, the first of the identities in Eq. (A.23) implies

$-2 \eta_{1 k}+4 \alpha_{11} \omega_{1 k}+4 \alpha_{21} \omega_{2 k}=0$

$-2 \eta_{2 k}+4 \alpha_{12} \omega_{1 k}+4 \alpha_{22} \omega_{2 k}=0$.

Since $\alpha_{12}=\alpha_{21}$, the preceding equations imply a key relation between the period matrices of the differentials $\left\{d u_{1}, d u_{2}\right\}$ and $\left\{d r_{1}, d r_{2}\right\}$ which will be essential in defining the Kleinian sigma function, viz.,

$\alpha=\frac{1}{2} \eta \omega^{-1}$

\section{A.2. Riemann theta functions}

The Kleinian sigma function will be defined using the Riemann theta function associated with the period matrix $\tau$ of the normalized differentials $d v_{1}$ and $d v_{2}$.

Definition 4. The theta function of $v \in \mathbb{C}^{2}$, associated with the period matrix $\tau$, is

$$
\begin{aligned}
\theta(v) & =\sum_{n_{1}=-\infty}^{\infty} \sum_{n_{2}=-\infty}^{\infty} e^{2 \pi i\left(v_{1} n_{1}+v_{2} n_{2}\right)+\pi i\left(\tau_{11} n_{1}^{2}+2 \tau_{12} n_{1} n_{2}+\tau_{22} n_{2}^{2}\right)} \\
& =\sum_{n=-\infty}^{\infty} e^{2 \pi i n v+\pi i n \tau n}
\end{aligned}
$$

with quasiperiodicity on the period lattice $\mathbb{C}^{2} /(I \oplus \tau)$ given by

$\theta\left(v+m+\tau m^{\prime}\right)=e^{-2 \pi i m^{\prime}\left(v+\frac{1}{2} \tau m^{\prime}\right)} \theta(v)$,

where $m, m^{\prime} \in \mathbb{Z}^{2}$.

It is a well-known fact that, since $\tau$ has a positive definite imaginary part, the Riemann theta function $\theta(v)$ is an entire function of $v \in \mathbb{C}^{2}$. Moreover, $\theta(v)$ is not identically zero, since it has non-vanishing Fourier coefficients in the special case in which it is considered as a Fourier series in the real variable $v \in \mathbb{R}^{2}$.

Definition 5. The partial derivatives of $\theta$ are denoted by

$\theta_{1}(v)=\frac{\partial \theta}{\partial v_{1}}(v)$,
$\theta_{2}(v)=\frac{\partial \theta}{\partial v_{2}}(v)$.
Moreover, the logarithmic derivatives of $\theta$ possess lattice transformations of the form, for $j=1,2$,

$$
\frac{\partial \ln \theta}{\partial v_{j}}\left(v+m+\tau m^{\prime}\right)=-2 \pi i m_{j}^{\prime}+\frac{\partial \ln \theta}{\partial v_{j}}(v) \text {. }
$$

Lemma 26. The choice of the canonical cycles obeying the conjugate relations (A.2) implies that the real part of $\tau$ satisfies Eq. (A.11) and, hence, it can be shown that

$\theta(v)^{*}=\theta\left(v^{*}\right)$.

Similarly, for $i=1,2$,

$\theta_{1}(v)^{*}=\theta_{1}\left(v^{*}\right)$

$\theta_{2}(v)^{*}=\theta_{2}\left(v^{*}\right)$.

Theorem 9. The theta function is zero at odd half-integer periods, viz., if $m, m^{\prime} \in \mathbb{Z}^{2}$ and $m m^{\prime}=m_{1} m_{1}^{\prime}+m_{2} m_{2}^{\prime}$ is an odd integer, then

$\theta\left(\frac{1}{2} m+\frac{1}{2} \tau m^{\prime}\right)=0$.

Proof.

$$
\begin{aligned}
\theta & \left(\frac{1}{2} m+\frac{1}{2} \tau m^{\prime}\right) \\
& =\sum_{n=-\infty}^{\infty} e^{\pi i m n+\pi i\left(n+\frac{1}{2} m^{\prime}\right) \tau\left(n+\frac{1}{2} m^{\prime}\right)-\frac{\pi i}{4} m^{\prime} \tau m^{\prime}}, \\
& =\sum_{n=-\infty}^{\infty} e^{-\pi i m n+\pi i\left(n-\frac{1}{2} m^{\prime}\right) \tau\left(n-\frac{1}{2} m^{\prime}\right)-\frac{\pi i}{4} m^{\prime} \tau m^{\prime}}, \\
& =e^{-i \pi m m^{\prime}} \sum_{n=-\infty}^{\infty} e^{-\pi i m\left(n-m^{\prime}\right)+\pi i\left(n-m^{\prime}+\frac{1}{2} m^{\prime}\right) \tau\left(n-m^{\prime}+\frac{1}{2} m^{\prime}\right)-\frac{\pi i}{4} m^{\prime} \tau m^{\prime}}, \\
& =e^{-i \pi m m^{\prime}} \sum_{n=-\infty}^{\infty} e^{-\pi i m n+\pi i\left(n+\frac{1}{2} m^{\prime}\right) \tau\left(n+\frac{1}{2} m^{\prime}\right)-\frac{\pi i}{4} m^{\prime} \tau m^{\prime}}, \\
& =e^{-i \pi m m^{\prime}} \sum_{n=-\infty}^{\infty} e^{-2 \pi i m n+\pi i m n+\pi i\left(n+\frac{1}{2} m^{\prime}\right) \tau\left(n+\frac{1}{2} m^{\prime}\right)-\frac{\pi i}{4} m^{\prime} \tau m^{\prime}} \\
& =e^{-i \pi m m^{\prime}} \sum_{n=-\infty}^{\infty} e^{\pi i m n+\pi i\left(n+\frac{1}{2} m^{\prime}\right) \tau\left(n+\frac{1}{2} m^{\prime}\right)-\frac{\pi i}{4} m^{\prime} \tau m^{\prime}}, \\
& =e^{-i \pi m m^{\prime}} \theta\left(\frac{1}{2} m+\frac{1}{2} \tau m^{\prime}\right),
\end{aligned}
$$

where the summation index is renamed, firstly, $n \rightarrow-n$ and, secondly, $n \rightarrow n-m^{\prime}$, without change to the sum, which is over all possible integer pairs $n \in \mathbb{Z}^{2}$.

Definition 6. The half-integer periods are denoted as

$$
\begin{aligned}
\frac{1}{2}\left[\begin{array}{ll}
m_{1}^{\prime} & m_{2}^{\prime} \\
m_{1} & m_{2}
\end{array}\right] & =\frac{1}{2}\left(\begin{array}{l}
m_{1}+\tau_{11} m_{1}^{\prime}+\tau_{12} m_{2}^{\prime} \\
m_{2}+\tau_{21} m_{1}^{\prime}+\tau_{22} m_{2}^{\prime}
\end{array}\right) \\
& =\frac{1}{2} m+\frac{1}{2} \tau m^{\prime},
\end{aligned}
$$

where $m, m^{\prime} \in \mathbb{Z}^{2}$.

Theorem 10. The fifteen integrals

$v^{\lambda_{i}, \lambda_{j}}=\int_{\lambda_{j}}^{\lambda_{i}} d v$ 
for $i, j=1, \ldots, 6$, with $i \neq j$, of the normalized holomorphic differentials $d v=\left(d v_{1}, d v_{2}\right)^{t}$ on the dissected Riemann surface $\mathscr{K}_{2}$ constructed from the canonical homology cycles in Fig. 7 between the fifteen pairs of distinct branch points of the Riemann surface $\mathscr{K}_{2}$ are equal to fifteen distinct nonzero half-integer periods, as follows,

$$
\begin{aligned}
& v^{\lambda_{1}, \lambda_{2}}=\frac{1}{2}\left[\begin{array}{ll}
0 & 0 \\
1 & 0
\end{array}\right], \quad v^{\lambda_{1}, \lambda_{3}}=\frac{1}{2}\left[\begin{array}{cc}
-1 & 1 \\
0 & -1
\end{array}\right], \\
& v^{\lambda_{1}, \lambda_{4}}=\frac{1}{2}\left[\begin{array}{cc}
-1 & 1 \\
0 & 0
\end{array}\right], \\
& v^{\lambda_{1}, \lambda_{5}}=\frac{1}{2}\left[\begin{array}{cc}
-1 & 0 \\
0 & -1
\end{array}\right], \quad v^{\lambda_{1}, \lambda_{6}}=\frac{1}{2}\left[\begin{array}{cc}
-1 & 0 \\
1 & 0
\end{array}\right], \\
& v^{\lambda_{2}, \lambda_{3}}=\frac{1}{2}\left[\begin{array}{cc}
-1 & 1 \\
-1 & -1
\end{array}\right], \\
& v^{\lambda_{2}, \lambda_{4}}=\frac{1}{2}\left[\begin{array}{cc}
-1 & 1 \\
-1 & 0
\end{array}\right], \quad v^{\lambda_{2}, \lambda_{5}}=\frac{1}{2}\left[\begin{array}{cc}
-1 & 0 \\
-1 & -1
\end{array}\right], \\
& v^{\lambda_{2}, \lambda_{6}}=\frac{1}{2}\left[\begin{array}{cc}
-1 & 0 \\
0 & 0
\end{array}\right], \\
& v^{\lambda_{3}, \lambda_{4}}=\frac{1}{2}\left[\begin{array}{cc}
0 & 0 \\
0 & 1
\end{array}\right], \\
& v^{\lambda_{3}, \lambda_{6}}=\frac{1}{2}\left[\begin{array}{cc}
0 & -1 \\
1 & 1
\end{array}\right], \quad v^{\lambda_{3}, \lambda_{5}}=\frac{1}{2}\left[\begin{array}{cc}
0 & -1 \\
0 & 0
\end{array}\right], \\
& v^{\lambda_{4}, \lambda_{5}}=\frac{1}{2}\left[\begin{array}{cc}
0 & -1 \\
0 & -1
\end{array}\right], \\
& v^{\lambda_{5}, \lambda_{6}}=\frac{1}{2}\left[\begin{array}{cc}
0 & 0 \\
1 & 1
\end{array}\right] .
\end{aligned}
$$

Proof. By examining the dissection of the Riemann surface $\mathscr{K}_{2}$ given by Fig. 7, it is possible to integrate between any two branch points on the dissected Riemann surface while remaining on the lower sheet of the two-sheeted covering, without crossing any of the basis cycles. However the same integral can be performed by tracing the same path projected on the upper sheet for which the integrand is the same except for multiplication by -1 due to the action of the hyperelliptic involution on $d v$. By keeping track of the crossings of the homology cycles on the upper sheet (so as to remain on the dissected Riemann surface), the equality of the two integration procedures leads to the stated results.

\section{Corollary 5.}

$$
\begin{aligned}
\theta\left(v^{\lambda_{1}, \lambda_{3}}\right) & =\theta\left(v^{\lambda_{1}, \lambda_{6}}\right)=\theta\left(v^{\lambda_{2}, \lambda_{4}}\right)=\theta\left(v^{\lambda_{2}, \lambda_{5}}\right)=\theta\left(v^{\lambda_{3}, \lambda_{6}}\right) \\
& =\theta\left(v^{\lambda_{4}, \lambda_{5}}\right)=0 .
\end{aligned}
$$

Proof. Theorem 9 states that the theta function is zero at the odd half-integer periods, which can be identified from the list in Theorem 10.

Lemma 27. If $\theta\left(\int_{\lambda_{6}}^{\lambda} d v\right)$ is not identically zero, then it has precisely two simple zeros at $\lambda=\lambda_{1}, \lambda_{3}$.

Proof. The existence of exactly two zeros follows from the standard technique of integrating the logarithmic derivative of $\theta\left(\int_{\lambda_{6}}^{\lambda} d v\right)$ around the edges of the dissected Riemann surface. The two zeros can then be identified from Corollary 5.

Lemma 28. $\theta\left(\int_{\lambda_{6}}^{\lambda} d v\right)$ is identically zero if and only if $\theta(0)=0$.

Proof. If $\theta(0)=0$, then $\theta\left(\int_{\lambda_{6}}^{\lambda} d v\right)$ has three zeros $\lambda=\lambda_{1}, \lambda_{3}$, and $\lambda=\lambda_{6}$. The result follows immediately from Lemma 27 .
Lemma 29. $\theta(0) \neq 0$ and, hence, $\theta\left(\int_{\lambda_{6}}^{\lambda} d v\right)$ is not identically zero. Proof. By way of contradiction, assume that $\theta(0)=0$, then it can be shown (see Baker [27, pp. 33-34]) that if $\theta\left(\int_{P}^{\lambda} d v\right)$ is identically zero for all $\lambda, P \in \mathscr{K}_{2}$, then $\theta(v)$ is itself identically zero as a function of $v \in \mathbb{C}^{2}$. Since $\theta(v)$ is not identically zero, there must exist some $P_{0} \in \mathscr{K}_{2}$ such that $\theta\left(\int_{P_{0}}^{\lambda} d v\right)$ is not identically zero. However, by the same reasoning of Lemma 27, $\theta\left(\int_{P_{0}}^{\lambda} d v\right)$ has exactly two zeros, one of which is $\lambda=P_{0}$. By Lemma 28 and the evenness of $\theta(v)$, we see that $\lambda=\lambda_{6}$ is a second zero. However, Corollary 5 is symmetric with respect to the six branch points, which means that the results for $\lambda_{6}$ in Lemmas 27 and 28 have analogous statements for each of the branch points. This means that each of the six branch points of $\mathscr{K}_{2}$ is a zero of $\theta\left(\int_{P_{0}}^{\lambda} d v\right)$, but the function can only have two zeros unless it is identically zero, a contradiction. Therefore $\theta(0) \neq 0$. Finally, Lemma 28 shows that $\theta\left(\int_{\lambda_{6}}^{\lambda} d v\right)$ is not identically zero.

Lemma 30. If $z_{1} \neq z_{2}$, then $\theta\left(\int_{\lambda_{6}}^{\lambda} d v-\int_{\lambda_{1}}^{z_{1}} d v-\int_{\lambda_{3}}^{z_{2}} d v\right)$ has precisely two simple zeros at $\lambda=z_{1}, z_{2} \in \mathscr{K}_{2}$.

Proof. See Baker [27] and use the fact that $\theta\left(\int_{\lambda_{6}}^{\lambda} d v\right)$ is not identically zero.

Lemma 31. $\theta(e)=0$ if and only if $\lambda \in \mathscr{K}_{2}$ such that

$e=\int_{\lambda_{6}}^{\lambda} d v+\int_{\lambda_{3}}^{\lambda_{1}} d v$.

The set of $e \in \mathbb{C}^{2} /(I \oplus \tau)$ having this property is called the theta divisor $\Theta$. The theta divisor is a one-complex-dimensional subvariety of the two-complex-dimensional period lattice $\mathbb{C}^{2} /(I \oplus \tau)$.

Proof. See Baker [27] and use the fact that $\theta\left(\int_{\lambda_{6}}^{\lambda} d v\right)$ is not identically zero.

Lemma 32. Suppose e $\notin \Theta$, then $\theta\left(\int_{\lambda_{6}}^{\lambda} d v-e\right)$ is not identically zero and has precisely two zeros $\lambda=z_{1}, z_{2} \in \mathscr{K}_{2}$. Moreover, up to addition of integer multiples of periods,

$e=\int_{\lambda_{1}}^{z_{1}} d v+\int_{\lambda_{3}}^{z_{2}} d v$.

Proof. See Baker [27] and use the fact that $\theta\left(\int_{\lambda_{6}}^{\lambda} d v\right)$ is not identically zero.

Thus, with the exception of the one-complex-dimensional variety $e \in \Theta$, the points $\left(z_{1}, s_{1}\right),\left(z_{2}, s_{2}\right) \in \mathscr{R}$ that satisfy Eq. (A.37) may be viewed as well-defined functions of the independent variable $e \in \mathbb{C}^{2} /(I \oplus \tau)$.

\section{A.3. Kleinian elliptic functions}

Definition 7. The fundamental Kleinian sigma function $\sigma$ of $u \in$ $\mathbb{C}^{2}$ is

$\sigma(u)=e^{\frac{1}{2} u \eta \omega^{-1} u} \theta\left(\frac{1}{2} \omega^{-1} u\right)$,

with transformations on the period lattice $\mathbb{C}^{2} /\left(2 \omega \bigoplus 2 \omega^{\prime}\right)$ given by, for $r=1,2$,

$\sigma\left(u_{1}+2 \omega_{1 r}, u_{2}+2 \omega_{2 r}\right)=e^{2 \eta_{1 r}\left(u_{1}+\omega_{1 r}\right)+2 \eta_{2 r}\left(u_{2}+\omega_{2 r}\right)} \sigma(u)$,

$\sigma\left(u_{1}+2 \omega_{1 r}^{\prime}, u_{2}+2 \omega_{2 r}^{\prime}\right)=e^{2 \eta_{1 r}^{\prime}\left(u_{1}+\omega_{1 r}^{\prime}\right)+2 \eta_{2 r}^{\prime}\left(u_{2}+\omega_{2 r}^{\prime}\right)} \sigma(u)$. 
In general, if $m=\left(m_{1}, m_{2}\right)^{T}$ and $m^{\prime}=\left(m_{1}^{\prime}, m_{2}^{\prime}\right)^{T}$ are two couples of integers and

$\Omega_{m}=2 \omega m+2 \omega^{\prime} m^{\prime}, \quad H_{m}=2 \eta m+2 \eta^{\prime} m^{\prime}$,

then

$\sigma\left(u+\Omega_{m}\right)=e^{H_{m}\left(u+\frac{1}{2} \Omega_{m}\right)-i \pi m m^{\prime}} \sigma(u)$.

Note that the sigma function can be multiplied by a constant factor independent of $u$ without altering any of the results of this paper. The simplest normalization sufficient to accomplish the Jacobi inversion has been chosen. The definition of the Kleinian sigma function is motivated by the following identities between integrals on the Riemann surface.

Firstly, Eq. (A.20) implies

$$
\begin{aligned}
\mathscr{K} & \left(\lambda, a ; z_{1}, b_{1}\right)+\mathscr{K}\left(\lambda, a ; z_{2}, b_{2}\right) \\
& -\int_{a}^{\lambda} d \Pi_{z_{1}, b_{1}}-\int_{a}^{\lambda} d \Pi_{z_{2}, b_{2}} \\
= & -2 \sum_{i=1}^{2} \sum_{j=1}^{2} \alpha_{i j} \int_{a}^{\lambda} d u_{i}\left(\int_{b_{1}}^{z_{1}} d u_{j}+\int_{b_{2}}^{z_{2}} d u_{j}\right) .
\end{aligned}
$$

Secondly, the identity

$$
\begin{aligned}
& \int_{a}^{\lambda} d \Pi_{z_{1}, b_{1}}+\int_{a}^{\lambda} d \Pi_{z_{2}, b_{2}} \\
& \quad=\log \frac{\theta\left(\int_{\lambda_{6}}^{\lambda} d v-\int_{\lambda_{1}}^{z_{1}} d v-\int_{\lambda_{3}}^{z_{2}} d v\right) \theta\left(\int_{\lambda_{6}}^{a} d v-\int_{\lambda_{1}}^{b_{1}} d v-\int_{\lambda_{3}}^{b_{2}} d v\right)}{\theta\left(\int_{\lambda_{6}}^{\lambda} d v-\int_{\lambda_{1}}^{b_{1}} d v-\int_{\lambda_{3}}^{b_{2}} d v\right) \theta\left(\int_{\lambda_{6}}^{a} d v-\int_{\lambda_{1}}^{z_{1}} d v-\int_{\lambda_{3}}^{z_{2}} d v\right)},
\end{aligned}
$$

follows from considering the function

$$
\begin{aligned}
& \frac{\theta\left(\int_{\lambda_{6}}^{\lambda} d v-\int_{\lambda_{1}}^{z_{1}} d v-\int_{\lambda_{3}}^{z_{2}} d v\right)}{\theta\left(\int_{\lambda_{6}}^{\lambda} d v-\int_{\lambda_{1}}^{b_{1}} d v-\int_{\lambda_{3}}^{b_{2}} d v\right)} \\
& \quad \times \exp \left(-\int_{a}^{\lambda} d \Pi_{z_{1}, b_{1}}-\int_{a}^{\lambda} d \Pi_{z_{2}, b_{2}}\right),
\end{aligned}
$$

which must be constant because it has no zeros and no poles and zero periods around all homology basis cycles. Therefore it is equal to its value when $\lambda=a$. A similar conclusion follows even if only the periods around $\mathfrak{a}_{1}$ and $\mathfrak{a}_{2}$ are zero and the periods around $\mathfrak{b}_{1}$ and $\mathfrak{b}_{2}$ are constant (but not necessarily zero).

The two identities in Eqs. (A.42) and (A.43) can be written in terms of the Kleinian sigma function (A.38), using the fact that $\alpha=\frac{1}{2} \eta \omega^{-1}$, as

$$
\begin{aligned}
& \mathscr{K}\left(\lambda, a ; z_{1}, b_{1}\right)+\mathscr{K}\left(\lambda, a ; z_{2}, b_{2}\right) \\
& =\log \frac{\sigma\left(\int_{\lambda_{6}}^{\lambda} d u-\int_{\lambda_{1}}^{z_{1}} d u-\int_{\lambda_{3}}^{z_{2}} d u\right) \sigma\left(\int_{\lambda_{6}}^{a} d u-\int_{\lambda_{1}}^{b_{1}} d u-\int_{\lambda_{3}}^{b_{2}} d u\right)}{\sigma\left(\int_{\lambda_{6}}^{\lambda} d u-\int_{\lambda_{1}}^{b_{1}} d u-\int_{\lambda_{3}}^{b_{2}} d u\right) \sigma\left(\int_{\lambda_{6}}^{a} d u-\int_{\lambda_{1}}^{z_{1}} d u-\int_{\lambda_{3}}^{z_{2}} d u\right)},
\end{aligned}
$$

where $d u=\left(d u_{1}, d u_{2}\right)^{T}$ and $d v=\left(d v_{1}, d v_{2}\right)^{T}$.

Now Eq. (A.13), in which we interchange $(\lambda, a)$ and $(z, b)$ using the symmetry of the expression, and Eq. (A.17) imply

$$
\begin{aligned}
\mathscr{K}\left(\lambda, a ; z_{1}, b_{1}\right)+\mathscr{K}\left(\lambda, a ; z_{2}, b_{2}\right) \\
=\int_{b_{1}}^{z_{1}} \int_{a}^{\lambda} \frac{\partial}{\partial \lambda} \frac{s+w}{z-\lambda} \frac{d \lambda}{2 s} d z+\int_{b_{2}}^{z_{2}} \int_{a}^{\lambda} \frac{\partial}{\partial \lambda} \frac{s+w}{z-\lambda} \frac{d \lambda}{2 s} d z \\
\quad+\left(\int_{b_{1}}^{z_{1}} d u_{1}+\int_{b_{2}}^{z_{2}} d u_{1}\right) \int_{a}^{\lambda} d r_{1} \\
\quad+\left(\int_{b_{1}}^{z_{1}} d u_{2}+\int_{b_{2}}^{z_{2}} d u_{2}\right) \int_{a}^{\lambda} d r_{2} .
\end{aligned}
$$

The identities in Eqs. (A.44) and (A.45) combine to give

$$
\begin{aligned}
& \log \frac{\sigma\left(\int_{\lambda_{6}}^{\lambda} d u-\int_{\lambda_{1}}^{z_{1}} d u-\int_{\lambda_{3}}^{z_{2}} d u\right) \sigma\left(\int_{\lambda_{6}}^{a} d u-\int_{\lambda_{1}}^{b_{1}} d u-\int_{\lambda_{3}}^{b_{2}} d u\right)}{\sigma\left(\int_{\lambda_{6}}^{\lambda} d u-\int_{\lambda_{1}}^{b_{1}} d u-\int_{\lambda_{3}}^{b_{2}} d u\right) \sigma\left(\int_{\lambda_{6}}^{a} d u-\int_{\lambda_{1}}^{z_{1}} d u-\int_{\lambda_{3}}^{z_{2}} d u\right)} \\
& =\int_{b_{1}}^{z_{1}} \int_{a}^{\lambda} \frac{\partial}{\partial \lambda} \frac{s+w}{z-\lambda} \frac{d \lambda}{2 s} d z+\int_{b_{2}}^{z_{2}} \int_{a}^{\lambda} \frac{\partial}{\partial \lambda} \frac{s+w}{z-\lambda} \frac{d \lambda}{2 s} d z \\
& \quad+\left(\int_{b_{1}}^{z_{1}} d u_{1}+\int_{b_{2}}^{z_{2}} d u_{1}\right) \int_{a}^{\lambda} d r_{1} \\
& \quad+\left(\int_{b_{1}}^{z_{1}} d u_{2}+\int_{b_{2}}^{z_{2}} d u_{2}\right) \int_{a}^{\lambda} d r_{2} .
\end{aligned}
$$

Lemma 32 and the discussion following Eq. (A.43) shows that in Eq. (A.46), for $u^{\prime}, u^{\prime \prime} \notin \Theta$, we can write

$u^{\prime}=\int_{\lambda_{1}}^{z_{1}} d u+\int_{\lambda_{3}}^{z_{2}} d u, \quad u^{\prime \prime}=\int_{\lambda_{1}}^{b_{1}} d u+\int_{\lambda_{3}}^{b_{2}} d u$,

consider $z_{1}$ and $z_{2}$ as functions of $u^{\prime}$ and $b_{1}$ and $b_{2}$ as functions of $u^{\prime \prime}$. Thus Eq. (A.46) becomes,

$$
\begin{aligned}
& \log \frac{\sigma\left(\int_{\lambda_{6}}^{\lambda} d u-u^{\prime}\right) \sigma\left(\int_{\lambda_{6}}^{a} d u-u^{\prime \prime}\right)}{\sigma\left(\int_{\lambda_{6}}^{\lambda} d u-u^{\prime \prime}\right) \sigma\left(\int_{\lambda_{6}}^{a} d u-u^{\prime}\right)} \\
& =\int_{b_{1}}^{z_{1}} \int_{a}^{\lambda} \frac{\partial}{\partial \lambda} \frac{s+w}{z-\lambda} \frac{d \lambda}{2 s} d z+\int_{b_{2}}^{z_{2}} \int_{a}^{\lambda} \frac{\partial}{\partial \lambda} \frac{s+w}{z-\lambda} \frac{d \lambda}{2 s} d z \\
& \quad+\left(u_{1}^{\prime}-u_{1}^{\prime \prime}\right) \int_{a}^{\lambda} d r_{1}+\left(u_{2}^{\prime}-u_{2}^{\prime \prime}\right) \int_{a}^{\lambda} d r_{2} .
\end{aligned}
$$

Differentiation of Eq. (A.47) with respect to $u_{i}^{\prime}$ for $i=1,2$, produces

$$
\begin{gathered}
-\zeta_{i}\left(\int_{\lambda_{6}}^{\lambda} d u-u^{\prime}\right)+\zeta_{i}\left(\int_{\lambda_{6}}^{a} d u-u^{\prime}\right) \\
=\frac{\partial z_{1}}{\partial u_{i}^{\prime}} \int_{a}^{\lambda} \frac{\partial}{\partial \lambda} \frac{s_{1}+w}{z_{1}-\lambda} \frac{d \lambda}{2 s_{1}} \\
+\frac{\partial z_{2}}{\partial u_{i}^{\prime}} \int_{a}^{\lambda} \frac{\partial}{\partial \lambda} \frac{s_{2}+w}{z_{2}-\lambda} \frac{d \lambda}{2 s_{2}}+\int_{a}^{\lambda} d r_{i},
\end{gathered}
$$

where the functions $\zeta_{i}$ and $\wp_{i j}$ for $i, j=1,2$, are defined by

$\zeta_{i}(u)=\frac{\partial}{\partial u_{i}} \log \sigma(u), \quad \wp_{i j}=-\frac{\partial^{2}}{\partial u_{i} \partial u_{j}} \log \sigma(u)$.

Eq. (A.41) shows that, for $i, j=1,2$, and $\Omega_{m}$ an integer translation across the period lattice of the Jacobi variety of $\mathscr{K}_{2}$,

$$
\begin{aligned}
\zeta_{i}\left(u+\Omega_{m}\right)-\zeta_{i}(u)= & \left(H_{m}\right)_{i}=2 \eta_{i 1} m_{1}+2 \eta_{i 2} m_{2} \\
& +2 \eta_{i 1}^{\prime} m_{1}^{\prime}+2 \eta_{i 2}^{\prime} m_{2}^{\prime}
\end{aligned}
$$

and

$\wp_{i j}\left(u+\Omega_{m}\right)=\wp_{i j}(u)$.

Using

$u_{1}=\int_{\lambda_{1}}^{z_{1}} \frac{d z}{s}+\int_{\lambda_{3}}^{z_{2}} \frac{d z}{s}$,
$u_{2}=\int_{\lambda_{1}}^{z_{1}} \frac{z d z}{s}+\int_{\lambda_{3}}^{z_{2}} \frac{z d z}{s}$,

we find that

$\frac{\partial z_{1}}{\partial u_{1}}=\frac{s_{1} z_{2}}{z_{2}-z_{1}}, \frac{\partial z_{2}}{\partial u_{1}}=\frac{s_{2} z_{1}}{z_{1}-z_{2}}$,
$\frac{\partial z_{1}}{\partial u_{2}}=\frac{s_{1}}{z_{1}-z_{2}}, \frac{\partial z_{2}}{\partial u_{2}}=\frac{s_{2}}{z_{2}-z_{1}}$. 
In Eq. (A.48) we now make the change of replacing the points $\left(z_{1}, s_{1}\right),\left(z_{2}, s_{2}\right) \in \mathscr{K}_{2}$ with their corresponding points under the hyperelliptic involution, viz., $\left(z_{1},-s_{1}\right)$ and $\left(z_{2},-s_{2}\right)$. Notice that $u_{r}^{\prime}$ is changed by this transformation to

$$
-u_{r}^{\prime}+2 \omega_{i 1} m_{1}+2 \omega_{i 2} m_{2}+2 \omega_{i 1}^{\prime} m_{1}^{\prime}+2 \omega_{i 2}^{\prime} m_{2}^{\prime}
$$

for some integers $m_{1}, m_{2}, m_{1}^{\prime}, m_{2}^{\prime}$. Making the corresponding change in the right-hand side of Eq. (A.48) and using Eq. (A.53), we obtain, for $i=1,2$,

$$
\begin{aligned}
& \int_{a}^{\lambda} d r_{i}+\zeta_{i}\left(\int_{\lambda_{6}}^{\lambda} d u+u^{\prime}\right)-\frac{1}{2} f_{i}\left(\lambda, z_{1}, z_{2}\right) \\
& =\zeta_{i}\left(\int_{\lambda_{6}}^{a} d u+u^{\prime}\right)-\frac{1}{2} f_{i}\left(a, z_{1}, z_{2}\right),
\end{aligned}
$$

where

$$
\begin{aligned}
f_{1}\left(\lambda, z_{1}, z_{2}\right)= & \frac{w\left(\lambda-z_{1}-z_{2}\right)}{\left(z_{1}-\lambda\right)\left(z_{2}-\lambda\right)}+\frac{s_{1}\left(z_{1}-\lambda-z_{2}\right)}{\left(z_{1}-\lambda\right)\left(z_{1}-z_{2}\right)} \\
& +\frac{s_{2}\left(z_{2}-\lambda-z_{1}\right)}{\left(z_{2}-\lambda\right)\left(z_{2}-z_{1}\right)}, \\
f_{2}\left(\lambda, z_{1}, z_{2}\right)= & \frac{s_{1}}{\left(z_{1}-\lambda\right)\left(z_{2}-\lambda\right)}+\frac{s_{2}}{\left(z_{1}-\lambda\right)\left(z_{1}-z_{2}\right)} \\
& +\frac{\left.z_{2}\right)}{\left(z_{2}-\lambda\right)\left(z_{2}-z_{1}\right)} .
\end{aligned}
$$

By adding $\int_{\lambda_{1}}^{z_{1}} d r_{i}+\int_{\lambda_{3}}^{z_{2}} d r_{i}$ to both sides of Eq. (A.55), we obtain

$$
\begin{aligned}
& \int_{a}^{\lambda} d r_{i}+\int_{\lambda_{1}}^{z_{1}} d r_{i}+\int_{\lambda_{3}}^{z_{2}} d r_{i} \\
& \quad+\zeta_{i}\left(\int_{\lambda_{6}}^{\lambda} d u+u^{\prime}\right)-\frac{1}{2} f_{i}\left(\lambda, z_{1}, z_{2}\right) \\
& =\int_{\lambda_{1}}^{z_{1}} d r_{i}+\int_{\lambda_{3}}^{z_{2}} d r_{i}+\zeta_{i}\left(\int_{\lambda_{6}}^{a} d u+u^{\prime}\right) \\
& \quad-\frac{1}{2} f_{i}\left(a, z_{1}, z_{2}\right),
\end{aligned}
$$

in which the left-hand side is symmetric with respect to $\lambda, z_{1}, z_{2}$, and the right-hand side is the value of the left-hand side when $\lambda=a$. Consequently the left-hand side of Eq. (A.57) is independent of $\lambda, z_{1}$, and $z_{2}$. Therefore, for $i=1,2$,

$$
\begin{aligned}
\zeta_{i}\left(\int_{\lambda_{6}}^{\lambda} d u+u^{\prime}\right)= & C_{i}+\frac{1}{2} f_{i}\left(\lambda, z_{1}, z_{2}\right)-\int_{a}^{\lambda} d r_{i} \\
& -\int_{\lambda_{1}}^{z_{1}} d r_{i}-\int_{\lambda_{3}}^{z_{2}} d r_{i},
\end{aligned}
$$

where $C_{i}$ is independent of $\lambda, z_{1}, z_{2}$. Since $a$ is an arbitrary point on $\mathcal{K}_{2}$, it can be set equal to the branch point at $a=\lambda_{6}$, giving

$$
\begin{aligned}
\zeta_{i}\left(\int_{\lambda_{6}}^{\lambda} d u+u^{\prime}\right)= & C_{i}+\frac{1}{2} f_{i}\left(\lambda, z_{1}, z_{2}\right)-\int_{\lambda_{6}}^{\lambda} d r_{i} \\
& -\int_{\lambda_{1}}^{z_{1}} d r_{i}-\int_{\lambda_{3}}^{z_{2}} d r_{i} .
\end{aligned}
$$

Now $\zeta_{i}(0)=0$, being an odd function, so setting $\lambda=\lambda_{6}, z_{1}=\lambda_{1}$, and $z_{2}=\lambda_{3}$, shows that $C_{i}=0$, for $i=1,2$.

Direct calculation shows that, as $\lambda \rightarrow \infty^{ \pm}$, the singularities in the terms on the right-hand side of Eq. (A.59) cancel out, so that

$$
\lim _{\lambda \rightarrow \infty^{ \pm}} \frac{1}{2} f_{i}\left(\lambda, z_{1}, z_{2}\right)-\int_{\lambda_{6}}^{\lambda} d r_{i}=\gamma_{i}^{ \pm},
$$

where

$$
\begin{aligned}
& \gamma_{1}^{ \pm}= \pm \frac{1}{2}\left(-z_{1} z_{2}-\frac{1}{8} \Lambda_{1}^{2}+\frac{1}{2} \Lambda_{2}\right)+\frac{1}{2} \frac{s_{1}-s_{2}}{z_{1}-z_{2}}+\delta_{1}^{ \pm}, \\
& \gamma_{2}^{ \pm}= \pm \frac{1}{2}\left(z_{1}+z_{2}-\frac{1}{2} \Lambda_{1}\right)+\delta_{2}^{ \pm},
\end{aligned}
$$

and $\delta_{1}^{ \pm}$and $\delta_{2}^{ \pm}$are constants independent of $z_{1}$ and $z_{2}$. Thus Eq. (A.59) becomes

$\zeta_{i}\left(\int_{\lambda_{6}}^{\infty^{ \pm}} d u+u^{\prime}\right)=\gamma_{i}^{ \pm}-\int_{\lambda_{1}}^{z_{1}} d r_{i}-\int_{\lambda_{3}}^{z_{2}} d r_{i}$.

Eq. (A.62), implies, for $i=1,2$,

$$
\zeta_{i}\left(u^{\prime}+\int_{\lambda_{6}}^{\infty^{+}} d u\right)-\zeta_{i}\left(u^{\prime}+\int_{\lambda_{6}}^{\infty^{-}} d u\right)=\gamma_{i}^{+}-\gamma_{i}^{-} .
$$

Substituting the explicit expressions for $\gamma_{i}^{ \pm}$, we obtain the solution to the Jacobi inversion problem,

$$
\begin{aligned}
& \zeta_{1}\left(u^{\prime}+\int_{\lambda_{6}}^{\infty^{+}} d u\right)-\zeta_{1}\left(u^{\prime}+\int_{\lambda_{6}}^{\infty^{-}} d u\right) \\
& =-z_{1} z_{2}-\frac{1}{8} \Lambda_{1}^{2}+\frac{1}{2} \Lambda_{2}+\delta_{1}, \\
& \zeta_{2}\left(u^{\prime}+\int_{\lambda_{6}}^{\infty^{+}} d u\right)-\zeta_{2}\left(u^{\prime}+\int_{\lambda_{6}}^{\infty^{-}} d u\right) \\
& =z_{1}+z_{2}-\frac{1}{2} \Lambda_{1}+\delta_{2},
\end{aligned}
$$

where the constants $\delta_{1}$ and $\delta_{2}$ are independent of $z_{1}$ and $z_{2}$, and so can be obtained from Eq. (A.64) by setting $z_{1}=\left(\lambda_{1}, 0\right)$ and $z_{2}=\left(\lambda_{2}, 0\right)=\left(\lambda_{1}^{*}, 0\right)$. Recall $\alpha_{i j}$ for $i, j=1,2$, is given by

$$
\alpha=\frac{1}{2} \eta \omega^{-1}=\left(\begin{array}{ll}
\alpha_{11} & \alpha_{12} \\
\alpha_{21} & \alpha_{22}
\end{array}\right)
$$

Hence

$$
\begin{aligned}
& \delta_{1}=\delta_{1}^{+}-\delta_{1}^{-}=-\left(-\left|\lambda_{1}\right|^{2}-\frac{1}{8} \Lambda_{1}^{2}+\frac{1}{2} \Lambda_{2}\right) \\
& +\zeta_{1}\left(\int_{\lambda_{6}}^{\infty^{+}} d u+\int_{\lambda_{3}}^{\lambda_{2}} d u\right) \\
& -\zeta_{1}\left(\int_{\lambda_{6}}^{\infty^{-}} d u+\int_{\lambda_{3}}^{\lambda_{2}} d u\right) \\
& =-\left(-\left|\lambda_{1}\right|^{2}-\frac{1}{8} \Lambda_{1}^{2}+\frac{1}{2} \Lambda_{2}\right)+2 \alpha_{11} \int_{\infty^{-}}^{\infty^{+}} d u_{1} \\
& +\left(\alpha_{12}+\alpha_{21}\right) \int_{\infty^{-}}^{\infty^{+}} d u_{2}+\frac{1}{2}\left(\omega^{-1}\right)_{11} \\
& \times\left(\frac{\theta_{1}^{+}}{\theta^{+}}-\frac{\theta_{1}^{-}}{\theta^{-}}\right)+\frac{1}{2}\left(\omega^{-1}\right)_{21}\left(\frac{\theta_{2}^{+}}{\theta^{+}}-\frac{\theta_{2}^{-}}{\theta^{-}}\right), \\
& \delta_{2}=\delta_{2}^{+}-\delta_{2}^{-}=-\left(\lambda_{1}+\lambda_{1}^{*}-\frac{1}{2} \Lambda_{1}\right) \\
& +\zeta_{2}\left(\int_{\lambda_{6}}^{\infty^{+}} d u+\int_{\lambda_{3}}^{\lambda_{2}}\right)-\zeta_{2}\left(\int_{\lambda_{6}}^{\infty^{-}} d u+\int_{\lambda_{3}}^{\lambda_{2}} d u\right) \\
& =-\left(\lambda_{1}+\lambda_{1}^{*}-\frac{1}{2} \Lambda_{1}\right)+\left(\alpha_{12}+\alpha_{21}\right) \int_{\infty^{-}}^{\infty^{+}} d u_{1} \\
& +2 \alpha_{22} \int_{\infty^{-}}^{\infty^{+}} d u_{2}+\frac{1}{2}\left(\omega^{-1}\right)_{12}\left(\frac{\theta_{1}^{+}}{\theta^{+}}-\frac{\theta_{1}^{-}}{\theta^{-}}\right) \\
& +\frac{1}{2}\left(\omega^{-1}\right)_{22}\left(\frac{\theta_{2}^{+}}{\theta^{+}}-\frac{\theta_{2}^{-}}{\theta^{-}}\right) \text {, }
\end{aligned}
$$


where

$$
\begin{aligned}
& \theta^{+}=\theta\left(\frac{1}{2} \omega^{-1}\left(\int_{\lambda_{6}}^{\infty^{+}} d u+\int_{\lambda_{3}}^{\lambda_{2}} d u\right)\right), \\
& \theta^{-}=\theta\left(\frac{1}{2} \omega^{-1}\left(\int_{\lambda_{6}}^{\infty^{-}} d u+\int_{\lambda_{3}}^{\lambda_{2}} d u\right)\right), \\
& \theta_{1}^{+}=\theta_{1}\left(\frac{1}{2} \omega^{-1}\left(\int_{\lambda_{6}}^{\infty^{+}} d u+\int_{\lambda_{3}}^{\lambda_{2}} d u\right)\right), \\
& \theta_{1}^{-}=\theta_{1}\left(\frac{1}{2} \omega^{-1}\left(\int_{\lambda_{6}}^{\infty^{-}} d u+\int_{\lambda_{3}}^{\lambda_{2}} d u\right)\right), \\
& \theta_{2}^{+}=\theta_{2}\left(\frac{1}{2} \omega^{-1}\left(\int_{\lambda_{6}}^{\infty^{+}} d u+\int_{\lambda_{3}}^{\lambda_{2}} d u\right)\right), \\
& \theta_{2}^{-}=\theta_{2}\left(\frac{1}{2} \omega^{-1}\left(\int_{\lambda_{6}}^{\infty^{-}} d u+\int_{\lambda_{3}}^{\lambda_{2}} d u\right)\right) .
\end{aligned}
$$

Explicit expressions for $z_{1}$ and $z_{2}$ can then be found from the quadratic formula for the roots of the quadratic equation

$z^{2}-\left(z_{1}+z_{2}\right) z+z_{1} z_{2}=0$.

The corresponding values [28] of $s_{1}$ and $s_{2}$ for $\left(z_{1}, s_{1}\right),\left(z_{2}, s_{2}\right) \in \mathscr{K}_{2}$ are, for $k=1,2$,

$$
\begin{aligned}
s_{k}= & \left(\wp_{22}\left(u^{\prime}+\int_{\lambda_{6}}^{\infty^{-}} d u\right)-\wp_{22}\left(u^{\prime}+\int_{\lambda_{6}}^{\infty^{+}} d u\right) z_{k}\right. \\
& +\wp_{12}\left(u^{\prime}+\int_{\lambda_{6}}^{\infty^{-}} d u\right)-\wp_{12}\left(u^{\prime}+\int_{\lambda_{6}}^{\infty^{+}} d u\right) .
\end{aligned}
$$

\section{References}

[1] A.M. Kamchatnov, Nonlinear Periodic Waves and their Modulations: An Introductory Course, World Scientific, Singapore, 2000.

[2] M. Bertola, A. Tovbis, Universality for the focusing nonlinear Schrödinger equation at the gradient catastrophe point: Rational breathers and poles of the tritronquee solution to painleve I, Comm. Pure Appl. Math. 66 (2013) 678-752.

[3] G.A. El, E.G. Khamis, A. Tovbis, Dam break problem for the focusing nonlinear Schrödinger equation and the generation of rogue waves, arXiv: $1505.01785 \mathrm{v} 1$ [nlin.PS].

[4] A.V.Porubov, D.F. Parker, Some general periodic solutions to coupled nonlinear Schrödinger equations, Wave Motion 29 (1999) 97-109.

[5] P.L. Christiansen, J.C. Eilbeck, V.Z. Enolskii, N.A. Kostov, Quasi-periodic and periodic solutions for coupled nonlinear Schrödinger equations of the Manakov type, Proc. R. Soc. Lond. A 456 (2000) 2263-2281.

[6] J.C. Eilbeck, V.Z. Enolskii, N.A. Kostov, Quasi-periodic and periodic solutions for vector nonlinear Schrödinger equations, J. Math. Phys. 41 (2000) 8236-8248.

[7] T. Woodcock, O.H. Warren, J.N. Elgin, Genus two finite gap solutions of the vector nonlinear Schrödinger equation, J. Phys. A 40 (2007) F355-F361.

[8] A. Kamchatnov, On periodic solutions and their modulations of the Manakov system, J. Phys. A 47 (2014) 145203.

[9] E.D. Belokolos, A.I. Bobenko, V.Z. Enol'skii, A.R. Its, V.B. Matveev, AlgebroGeometric Approach to Nonlinear Integrable Equations, Springer-Verlag, Berlin, 1994.

[10] V.P. Kotlyarov, Periodic problem for the Schrödinger nonlinear equation, in: Voprosy Matematicheskoi Fiziki i Funkcionalnogo Analiza, 1, Naukova Dumka, Kiev, 1976, pp. 121-131. English transl. in arXiv:1401.4445v1 [nlin:SI].
[11] A.R. Its, V.P. Kotlyarov, Explicit formulas for solutions of the Schrödinger nonlinear equation, Dokl. Akad. Nauk Ukr. SSR A10 (1976) 965-968. English transl. in arXiv: $1401.4445 \mathrm{v} 1$ [nlin:SI].

[12] A.R. Its, Inversion of hyperelliptic integrals and integration of nonlinear differential equations, in: Vestnik Leningrad University, N 7, in: Ser. Math. Mekh. Astr. vyp., vol. 2, 1976, pp. 39-46. English transl. in Vestnik Leningrad University Math. 9 (1981) 121-129.

[13] I.V. Čerednik, On the conditions of reality in finite-gap integration, Dokl. Akad Nauk SSSR 252 (5) (1980) 1104-1108.

[14] B.A. Dubrovin, Theta functions and non-linear equations, Russian Math. Surveys 36 (2) (1981) 11-92.

[15] B.A. Dubrovin, S.P. Novikov, Algebrogeometric Poisson brackets for real finiterange solutions of the sine-Gordon and nonlinear Schrödinger equations, Dokl. Akad. Nauk SSSR 267 (6) (1982) 1295-1300.

[16] B.A. Dubrovin, S.M. Natanzon, Real two-zone solutions of the sine-Gordon equation, Funct. Anal. Appl. 16 (1) (1982) 21-33.

[17] S.P. Novikov, Algebro-topological approach to reality problems. Real action variables in the theory of finite-gap solutions of the sine-Gordon equation, Zap. Naunchn. Sem. Leningrad. Otdel. Mat. Steklov. (LOMI) 133 (1984) 177-196. Differential geometry, Lie groups and mechanics VI.

[18] E. Previato, Hyperelliptic quasi-periodic and soliton-solutions of the nonlinear Schrödinger equation, Duke Math. J. 52 (1985) 329-377.

[19] P.G. Grinevich, S.P. Novikov, Real finite-zone solutions of the sine-Gordon equation: a formula for the topological charge, Russian Math. Surveys 56 (5) (2001) 980-981.

[20] F. Gesztesy, H. Holden, Soliton Equations and their Algebro-Geometric Solutions, Volume I: $(1+1)$-Dimensional Continuous Models, in: Cambridge Studies in Advanced Mathematics, vol. 79, Cambridge University Press, Cambridge, 2003.

[21] F. Gesztesy, H. Holden, J. Michor, G. Teschl, Soliton Equations and their Algebro-Geometric Solutions, Volume II: $(1+1)$-Dimensional Continuous Models, in: Cambridge Studies in Advanced Mathematics, vol. 114, Cambridge University Press, Cambridge, 2008.

[22] E.R. Tracy, H.H. Chen, Y.C. Lee, Study of quasiperiodic solutions of the nonlinear Schrödinger equation and the nonlinear modulational instability, Phys. Rev. Lett. 53 (1984) 218-221.

[23] E.R. Tracy, H.H. Chen, Nonlinear self-modulation: An exactly solvable model, Phys. Rev. A 37 (1988) 815-839.

[24] A.M. Kamchatnov, On improving the effectiveness of periodic solutions of the NLS and DNLS equations, J. Phys. A: Math. Gen. 23 (1990) 2945-2960.

[25] M.G. Forest, D.W. McLaughlin, Spectral theory for the periodic sineGordon equation: A concrete viewpoint, J. Math. Phys. 23 (1982) 1248-1277.

[26] N. Ercolani, M.G. Forest, The geometry of real Sine-Gordon wavetrains, Comm. Math. Phys. 99 (1985) 1-49.

[27] H.F. Baker, Multiply Periodic Functions, Cambridge University Press, Cambridge, 1907.

[28] J.C. Eilbeck, V.Z. Enolskii, H. Holden, The hyperelliptic $\zeta$-function and the integrable massive thirring model, Proc. R. Soc. Lond. Ser. A Math. Phys. Eng. Sci. 459 (2035) (2003) 1581-1610.

[29] O.C. Wright III, Effective integration of ultra-elliptic solutions of the focusing nonlinear Schrödinger equation, arXiv:1505.03120v2 [nlin:SI].

[30] H. Flaschka, A.C. Newell, T. Ratiu, Kac-Moody Lie algebras and soliton equations. 2. Lax equations associated with A1, Physica D 9 (3) (1983) 300-323. http://dx.doi.org/10.1016/0167-2789(83)90274-9.

[31] T. Piezas, Solving Solvable Sextics using Polynomial Decomposition, 2004. http://www.oocities/titus_piezas/Sextic.pdf downloaded October 4, 2012.

[32] A.R. Osborne, Nonlinear Ocean Waves and the Inverse Scattering Transform, Elsevier, Amsterdam, 2010.

[33] J. Frauendiener, C. Klein, Algebraic curves and Riemann surfaces in Matlab, in: A.I. Bobenko, C. Klein (Eds.), Computational Approach to Riemann Surfaces, in: Lecture Notes in Mathematics, vol. 2013, Springer-Verlag, Berlin, 2011 pp. $125-162$.

[34] B. Deconinck, M.S. Patterson, Computing with plane algebraic curves and Riemann surfaces: The algorithms of the maple package algcurves, in: A.I. Bobenko, C. Klein (Eds.), Computational Approach to Riemann Surfaces, in: Lecture Notes in Mathematics, vol. 2013, Springer-Verlag, Berlin, 2011 pp. 67-123.

[35] J.N. Elgin, V.Z. Enolski, A.R. Its, Effective integration of the nonlinear vector Schrödinger equation, Physica D 225 (2007) 127-152.

[36] O.C. Wright III, On elliptic solutions of a coupled nonlinear Schrödinger equation, Physica D 264 (2013) 1-16. 